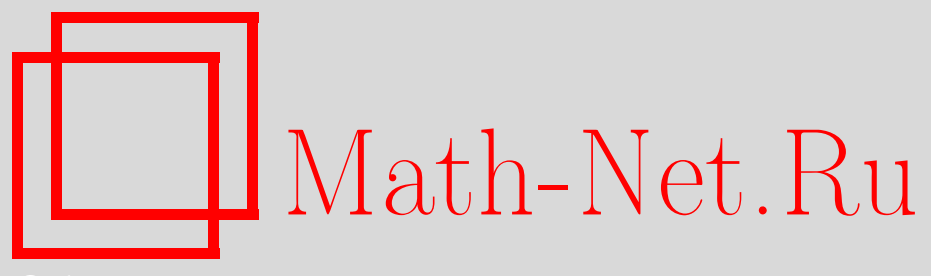

С. В. Асташкин, Ф. А. Сукочев, Независимые функции и геометрия банаховых пространств, УМH, 2010, том 65, выпуск 6, 3-86

DOI: https://doi.org/10.4213/rm9382

Использование Общероссийского математического портала Math-Net.Ru подразумевает, что вы прочитали и согласны с пользовательским соглашением http://www . mathnet.ru/rus/agreement

Параметры загрузки:

IP: 3.93.64.190

26 апреля 2023 г., 17:29:32

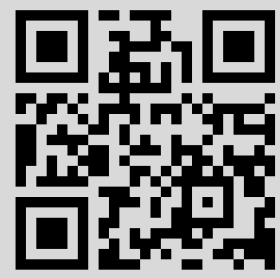




\title{
Независимые функции и геометрия банаховых пространств
}

\author{
С. В. Асташкин, Ф. А. Сукочев
}

\begin{abstract}
Основная цель этого обзора - дать представление о современном состоянии тех разделов теории независимых функций, которые связаны с вопросами геометрии функциональных пространств. "Величина" суммы независимых функций оценивается как в терминах классических моментов, так и в терминах норм симметричных пространств. Наибольшее внимание уделяется неравенству Розенталя и различным его обобщениям, границам их распространения на симметричные пространства. Центральная роль при этом принадлежит конструкции оператора Круглова, развитой в последние годы. В обзоре приведен также ряд приложений к геометрии банаховых пространств. В частности, рассматриваются варианты классических неравенств Морэ-Хинчина, изоморфизмы симметричных пространств на отрезке и полуоси, а также описание класса симметричных пространств, в которых любая последовательность симметрично и одинаково распределенных независимых случайных величин порождает гильбертово подпространство.
\end{abstract}

Библиография: 87 названий.

Ключевые слова: независимые функции, неравенства Хинчина, неравенства Розенталя, свойство Круглова, оператор Круглова, симметричное пространство, пространство Орлича, пространство Марцинкевича, пространство Лоренца, индексы Бойда, K-функционал, вещественный метод интерполяции, интегрально-равномерная норма.

\section{СоДЕРЖАНИЕ}

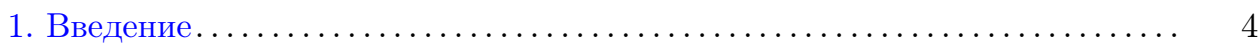

2. Определения, обозначения, предварительные сведения . . . . . . . . . . 7

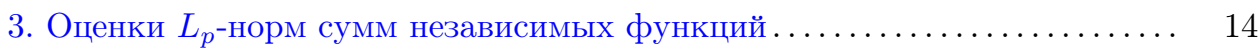

3.1. Неравенства Розенталя............................... 15

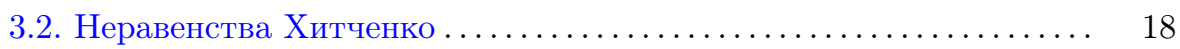

3.3. Теорема Латалы. .............................. 23

Первый автор поддержан Австралийским исследовательским советом и РФФИ (грант № 10-01-00077); второй автор поддержан Австралийским исследовательским советом.

(C) С.В. АСташкин, Ф.А. Сукочев, 2010 
4. Оператор Круглова в симметричных пространствах............. 26

4.1. Неравенства Розенталя и дизъюнктные суммы ............. 26

4.2. Свойство и оператор Круглова: первоначальные сведения . . . . . 27

4.3. Ограниченность оператора Круглова в симметричных прост-

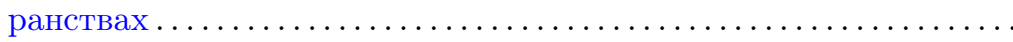

5. Сравнение сумм независимых и дизъюнктных функций в симметричных

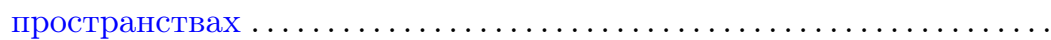

6. Неравенства типа Розенталя для симметричных (квази)норм на последовательностях независимых функций ................... 50

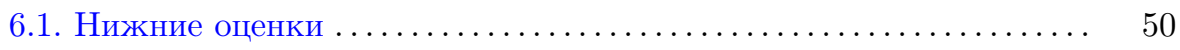

6.2. Верхние оценки в симметричных пространствах со свойством

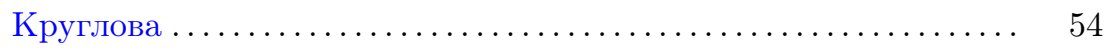

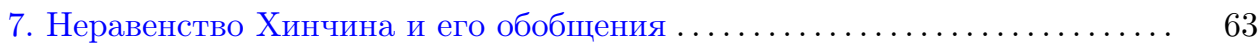

8. Оценки норм полиномов по системам независимых функций в простран-

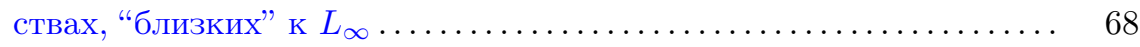

9. Изоморфизмы между симметричными пространствами на $[0,1]$ и $[0, \infty) 72$ 10. Гильбертовы подпространства симметричных пространств, порожден-

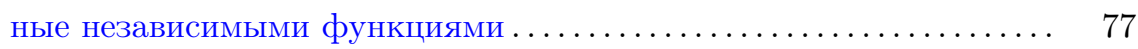

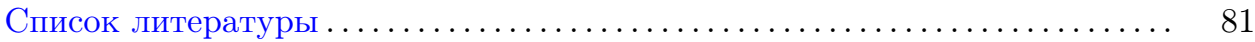

\section{1. Введение}

Последовательности независимых функций являются предметом изучения как теории вероятностей, так и теории функций и функционального анализа. Основная цель этого обзора - дать представление о современном состоянии тех разделов их теории, которые связаны с вопросами геометрии функциональных (прежде всего, симметричных) пространств. В центре нашего внимания будут неравенства, так или иначе связанные с описанием подпространств, порожденных последовательностями независимых функций в пространствах измеримых функций. Пионерской в этом отношении явилась работа Х. П. Розенталя [1], где были доказаны замечательные неравенства, позволившие описать изоморфные типы таких подпространств в $L_{p}$-пространствах. Хотелось бы также упомянуть две следующие тематически близкие обзорные статьи, опубликованные ранее в "Успехах математических наук". В первой из них [2], написанной В.Ф. Гапошкиным в 1966 г., в центре внимания находятся теоретико-функциональные свойства последовательностей как независимых, так и слабо зависимых (лакунарных) систем функций (сходимость и абсолютная сходимость, интегрируемость, предельные теоремы, закон повторного логарифма и т. д.). Второй обзор [3], авторы которого - Г. Пешкир и А. Н. Ширяев, написан гораздо позднее и посвящен изучению важнейшей и в то же время наиболее простой из систем независимых функций - системы Радемахера, возможности переноса ее свойств на общие мартингальные последовательности. Отметим, что изучению этой же системы, но только с точки зрения ее поведения в симметричных функциональных пространствах, посвящена также недавно опубликованная книга [4]. 
Речь в обзоре будет идти, в основном, о следующей задаче: предположим, что $\left\{f_{k}\right\}_{k=1}^{\infty}-$ последовательность независимых случайных величин (с. в.) на некотором вероятностном пространстве. Требуется каким-то образом оценить "величину" сумм $S_{n}=\sum_{k=1}^{n} f_{k}(n=1,2, \ldots)$. В случае, когда последовательность $\left\{f_{k}\right\}_{k=1}^{\infty}$ состоит из одинаково распределенных с. в. с нулевым математическим ожиданием и конечной дисперсией $\sigma^{2}>0$, эта проблема, разумеется, восходит к основаниям теории вероятностей. Давно известно, что при этих условиях пределом вероятностей $\mathrm{P}\left\{S_{n} /(\sigma \sqrt{n})>\tau\right\}$ при $n \rightarrow \infty$ будет стандартное гауссовское распределение. Обобщениям и уточнениям этого фундаментального факта посвящено необозримое количество статей и монографий (см. любой достаточно развернутый курс теории вероятностей). Наш подход иной: нас будут интересовать не асимптотические, а приближенные решения этой задачи. При этом в качестве "меры" $\left|S_{n}\right|$ будет рассматриваться не "хвост" распределения суммы, т. е. величина $\mathrm{P}\left\{\left|S_{n}\right|>\tau\right\}$ (его оценкам также посвящено большое количество исследований), а норма $\left\|S_{n}\right\|_{X}$ в том или ином функциональном пространстве $X$. Прежде всего, конечно, с этой точки зрения важна информация о классических моментах сумм $\left\|S_{n}\right\|_{p}=\left(\mathrm{E}\left|S_{n}\right|^{p}\right)^{1 / p}(p>0)$. В этой связи возникает задача найти величину $A_{n}$ (как можно более простую) такую, что для некоторой константы $C>0$ выполнено:

$$
C^{-1} A_{n} \leqslant\left\|S_{n}\right\|_{p} \leqslant C A_{n} \quad(p>0)
$$

Этот вопрос будет в центре нашего внимания в первой части обзора (раздел 3 ).

Хорошо известно, насколько отличается поведение сумм независимых функций в пространствах $L_{p}$ при конечных $p$ и в пространстве $L_{\infty}$. При этом происходящим явлениям, как правило, нельзя дать объяснение, находясь "внутри" $L_{p}$-шкалы. В то же время это можно сделать, выйдя за ее пределы и рассматривая общие симметричные пространства функций. Об этом пойдет речь во второй, главной части обзора (разделы 4-6). Нас, в основном, будут интересовать обобщения неравенства Розенталя (см. п. 3.1), границы его распространения на симметричные пространства. Как мы увидим в дальнейшем, последнее напрямую связано с возможностью сравнения сумм независимых и дизъюнктных функций. Центральное место занимает здесь конструкция так называемого оператора Круглова, которая была введена авторами обзора и имеет своим истоком одноименное свойство симметричного пространства, принадлежащее М.Ш. Браверману. Этот подход позволил использовать многочисленные преимущества операторного языка, например, применять теорию интерполяции операторов. В третьей и последней части обзора (разделы 7-10) рассматриваются приложения к вопросам геометрии симметричных пространств. Так, в терминах свойств оператора Круглова дана характеризация симметричных пространств, в которых справедлив вариант неравенства Хинчина, найдены наиболее широкие на сегодняшний день достаточные условия, при которых симметричные пространства на $[0,1]$ и $(0, \infty)$ изоморфны между собой, решен ряд других задач. 
Упомянем о еще двух важных направлениях, напрямую относящихся к данному обзору, но не включенных в него в связи с ограниченностью места. Первое направление достаточно полно отражено в работе [5], где устанавливается прямая связь между ограниченностью оператора Круглова и ограниченностью оператора случайных перестановок, естественным образом описывающего хорошо известные неравенства С. Квапеня и К. Шютта [6], [7]. Второе относится к некоммутативной теории вероятностей, когда измеримые независимые функции заменяются соответствующими последовательностями операторов. Мы отсылаем читателя к работе [8], где получены некоммутативные неравенства Розенталя. Библиографии в [5] и [8] дают, на наш взгляд, неплохое представление о развитии указанных направлений.

Изложим здесь же наиболее важные классические результаты, ставшие стимулом для дальнейших исследований. В 1923 г. А.Я. Хинчин в работе "Über dyadische Brüche" [9] доказал свои знаменитые неравенства, которые мы приведем в форме, принятой в теории функций, используя функции Радемахера $r_{n}(t)=\operatorname{sign} \sin 2^{n} \pi t, 0 \leqslant t \leqslant 1 \quad(n \in \mathbb{N})$.

Теорема 1 (неравенства Хинчина). Для любого $0<p<\infty$ существуют такие константы $A_{p}>0 u B_{p}>0$, что для всех $n \in \mathbb{N}$ и произвольных $a=\left(a_{k}\right)_{k=1}^{n} \in \mathbb{R}^{n}$ выполнено неравенство

$$
A_{p}\|a\|_{2} \leqslant\left\|\sum_{k=1}^{n} a_{k} r_{k}\right\|_{p} \leqslant B_{p}\|a\|_{2}
$$

где, как обычно, $\|f\|_{p}-$ норма в пространстве $L_{p}[0,1], a\|a\|_{2}=\left(\sum_{k}\left|a_{k}\right|^{2}\right)^{1 / 2}$.
При этом $B_{p} \leqslant \sqrt{p}(p \geqslant 1)$ и $B_{p} \sim \sqrt{p / e}$ при $p \rightarrow \infty$.

Эта теорема показывает, что $L_{p}$-нормы полиномов по системе Радемахера эквивалентны нормам последовательностей их коэффициентов в $l_{2}$. С точки зрения геометрии пространств последнее означает, что система Радемахера эквивалентна в $L_{p}[0,1](0<p<\infty)$ каноническому базису в $l_{2}$. Классическое доказательство теоремы 1 можно найти, например, в монографии [10; 4.5$]$ или в самом начале обзора [3]. Обобщением неравенств Хинчина явились неравенства Марцинкевича-Зигмунда [11], опубликованные в 1937 г. (позднее было доказано, что при выполнении условий следующей теоремы система независимых функций даже эквивалентна системе Радемахера по распределению [4; теорема 8.4 и следствие 8.3]).

Теорема 2 (неравенства Марцинкевича-Зигмунда). Пусть $\left\{f_{k}\right\}_{k=1}^{\infty}-c u$ стема независимых функций на $[0,1]$, удовлетворяющая условиям:

$$
\left\|f_{k}\right\|_{2}=1, \quad\left\|f_{k}\right\|_{\infty} \leqslant M, \quad \int_{0}^{1} f_{k}(t) d t=0 \quad(k \in \mathbb{N}) .
$$

Тогда для каждого $p \geqslant 1$ существует константа $C_{M, p}>0$, зависящая лишь от $M$ и р, такая, что для любого $n \in \mathbb{N}$ и произвольньх $a=\left(a_{k}\right)_{k=1}^{n} \in \mathbb{R}^{n}$ выполнено:

$$
C_{M, p}^{-1}\|a\|_{2} \leqslant\left\|\sum_{k=1}^{n} a_{k} f_{k}\right\|_{p} \leqslant C_{M, p}\|a\|_{2}
$$


Последняя теорема - простое следствие доказанной в [11] (см. также [12; теорема 2.5]) экспоненциальной оценки для распределения полиномов по системе независимых функций, имеющей свойства (2).

ТЕОРема 3. Если система независимых функиий $\left\{f_{k}\right\}_{k=1}^{\infty}$ удовлетворяет условиям (2), то для любых $n \in \mathbb{N}, a_{k} \in \mathbb{R} u \tau>0$ справедливо неравенство

$$
\lambda\left\{t \in[0,1]:\left|\sum_{k=1}^{n} a_{k} f_{k}(t)\right|>\tau\|a\|_{2}\right\} \leqslant 2 e^{-\tau^{2} /\left(4 M^{2}\right)} .
$$

Заметим, что значительно раньше, в 1929 г., оценки распределений сумм независимых функций, подобные (4), были доказаны А. Н. Колмогоровым [13]. При этом в отличие от предыдущих результатов они справедливы не только для систем равномерно ограниченных функций.

Теорема 4 (неравенства Колмогорова). Пусть $\left\{f_{k}\right\}_{k=1}^{\infty}$ - система ограниченных независимых с.в. таких, что $\mathrm{E} f_{k}=0(k=1,2, \ldots)$. Обозначим

$$
m_{k}=\left\|f_{k}\right\|_{\infty}, \quad M_{n}=\max _{k=1, \ldots, n} m_{k}, \quad b_{k}=\mathrm{E} f_{k}^{2}, \quad B_{n}=\sum_{k=1}^{n} b_{k} .
$$

Тогда для любых $n \in \mathbb{N} u \tau>0$

$$
\lambda\left\{t \in[0,1]: \sum_{k=1}^{n} f_{k}(t)>\tau\right\} \leqslant e^{-\tau /\left(4 B_{n}\right)}, \quad \text { если } \tau \geqslant \frac{B_{n}}{M_{n}},
$$

$u$

$$
\lambda\left\{t \in[0,1]: \sum_{k=1}^{n} f_{k}(t)>\tau\right\} \leqslant e^{-\tau^{2} /\left(4 B_{n}\right)}, \quad \text { если } \tau \leqslant \frac{B_{n}}{M_{n}} .
$$

Позднее, в 1959 г., Ю. В. Прохоров [14] доказал следующее замечательное усиление этих неравенств.

Теорема 5 (неравенство Прохорова). Пусть выполнены условия предыдущей теоремы. Тогда в тех же обозначениях справедливо неравенство

$$
\lambda\left\{\sum_{k=1}^{n} f_{k}>t\right\} \leqslant \exp \left(-\frac{t}{2 M_{n}} \operatorname{arcsh} \frac{t M_{n}}{2 B_{n}}\right) .
$$

\section{2. Определения, обозначения, предварительные сведения}

Пусть $(T, \Sigma, \mu)$ - пространство с $\sigma$-конечной мерой $\mu$, заданной на $\sigma$-алгебре $\Sigma$ подмножеств множества $T$. Совокупность всех почти всюду (п. в.) конечных измеримых вещественнозначных функций (классов эквивалентности) на $T$ с естественными алгебраическими операциями и топологией сходимости по мере $\mu$ на множествах конечной меры является линейным топологическим пространством, которое мы будем обозначать через $S(T, \Sigma, \mu)$. Как обычно, функции, совпадающие почти всюду, отождествляются, а запись $x \leqslant y(x, y \in$ $S(T, \Sigma, \mu))$ означает, что $x(t) \leqslant y(t)$ при почти всех $t \in T$. Далее нас будут 
интересовать, главным образом, случаи, когда $T$ - это отрезок $[0,1]$ с мерой Лебега $\lambda, n$-мерный $[0,1]^{n}(n \in \mathbb{N})$ или бесконечномерный $[0,1]^{\infty}$ куб с мерой $\prod_{k=1}^{n} \lambda_{k}$ или $\prod_{k=1}^{\infty} \lambda_{k}$ соответственно $\left(\lambda_{k}-\right.$ мера Лебега на $\left.[0,1]\right)$, а также полуось $(0, \infty)$ с мерой Лебега. Во всех случаях, кроме последнего, мы получаем вероятностные пространства, изоморфные между собой. Так, например, в случае $[0,1]$ и $[0,1]^{n}$ это означает, что существует сохраняющее меру отображение $\delta$, действующее из $[0,1]$ на $[0,1]^{n}$. Пространство $S(T, \Sigma, \mu)$ в этих случаях будет обозначаться через $S(0,1), S\left([0,1]^{n}\right), S\left([0,1]^{\infty}\right)$ и $S(0, \infty)$ соответственно. Всюду в дальнейшем $\chi_{B}(t)$ обозначает характеристическую функцию множества $B$, т. е. $\chi_{B}(t)=1$, если $t \in B$, и $\chi_{B}(t)=0$, если $t \notin B$.

Основной объект обзора - последовательности независимых функций, а "арена", на которой они рассматриваются - симметричные (перестановочно инвариантные) пространства на $[0,1]$ и $(0, \infty)$. Далее нам понадобятся лишь самые элементарные факты из теории вероятностей.

ОПРЕДЕЛЕНИЕ 1. Набор случайных величин (с. в.) $\left\{f_{k}\right\}_{k=1}^{n}$, определенных на вероятностном пространстве $(\Omega, \Sigma, \mathrm{P})$, называется независимым, если для любых интервалов $I_{k}$ на прямой $\mathbb{R}$ справедливо равенство

$$
\mathrm{P}\left\{\omega \in \Omega: f_{k}(\omega) \in I_{k}, k=1, \ldots, n\right\}=\prod_{k=1}^{n} \mathrm{P}\left\{\omega \in \Omega: f_{k}(\omega) \in I_{k}\right\} .
$$

Последовательность с.в. $\left\{f_{k}\right\}_{k=1}^{\infty}$ называют независимой, если для каждого $n \in \mathbb{N}$ набор $\left\{f_{k}\right\}_{k=1}^{n}$ независим.

Напомним также, что с. в. $f$ называется симметрично распределенной, если величины $f$ и $-f$ одинаково распределены. Через $\mathscr{F}_{f}(x)$ в дальнейшем будет обозначаться (традиционная для теории вероятностей) функция распределения, а через $\theta_{f}(t)$ - характеристическая функция с. в. $f$, т. е. $\theta_{f}(t)=$ $\int_{-\infty}^{\infty} e^{i t x} d \mathscr{F}_{f}(x)$

Пусть $(\Omega, \Sigma, \mathrm{P})$ - вероятностное пространство, $f$ - интегрируемая с. в., определенная на нем. Если $\mathfrak{R}-\sigma$-подалгебра $\sigma$-алгебры $\Sigma$, то существует и единственна (с точностью до меры нуль) $\mathfrak{R}$-измеримая интегрируемая с. в. $\mathrm{E}_{\mathfrak{R}} f$, которая для всех $B \in \mathfrak{R}$ удовлетворяет равенству

$$
\int_{B} f(\omega) d \mathrm{P}(\omega)=\int_{B} \mathrm{E}_{\mathfrak{R}} f(\omega) d \mathrm{P}(\omega)
$$

[15; теорема 1.1]. Случайную величину $\mathrm{E}_{\mathfrak{R}} f$ называют условным математическим ожиданием $f$ относительно $\sigma$-алгебры $\mathfrak{R}$. Кроме того, как обычно, $\mathrm{E} f=\int_{\Omega} f(\omega) d \mathrm{P}(\omega)$

Понятия и результаты из теории симметричных пространств будут использоваться гораздо интенсивнее. Поэтому мы уделим ей бо́льшее внимание, ограничившись, в основном, пространствами функций, определенных на $[0,1]$ (подробнее см. монографии [16]-[18]). 
Пусть $J=[0,1]$ или $(0, \infty)$. Для функции $x=x(t) \in S(J), x \geqslant 0$, введем функцию распределения, принятую в теории функций: $n_{x}(\tau):=\lambda\{t \in J$ : $x(t)>\tau\} \quad(\tau>0)$. Она неотрицательна, не возрастает и непрерывна справа. Две неотрицательные функции $x, y \in S(J)$ называются равноизмеримыми, если $n_{x}(\tau)=n_{y}(\tau)(\tau>0)$. В частности, любая функция $x \in S(J), x \geqslant 0$, равноизмерима со своей невозрастающей непрерывной слева перестановкой

$$
x^{*}(t):=\inf \left\{\tau \geqslant 0: n_{x}(\tau)<t\right\} \quad(t \in J) .
$$

Для произвольной $x(t) \in S(J)$ через $x^{*}(t)$ обозначается перестановка функции $|x(t)|$.

ОПРЕДЕЛЕНИЕ 2. Банахово функциональное пространство $X \subset S(J)$ называется симметричным, если: 1) из того, что $x \in X, y \in S(J)$ и $|y(t)| \leqslant|x(t)|$ п. в., следует, что $y \in X$ и $\left.\|y\|_{X} \leqslant\|x\|_{X} ; 2\right)$ из того, что $x \in X, y \in S(J)$ и функции $|x(t)|$ и $|y(t)|$ равноизмеримы, следует, что $y \in X$ и $\|y\|_{X}=\|x\|_{X}$.

Любое симметричное пространство $X$ на $J$ линейно и непрерывно вложено в отделимое линейное топологическое пространство $S(J)$ [19; теорема 4.3.1]. Это означает, в частности, что из сходимости $\left\|x_{n}-x\right\|_{X} \rightarrow 0\left(x_{n}, x \in X\right)$ следует сходимость $x_{n} \rightarrow x$ по мере $\lambda$ на множествах конечной меры из $J$. Если $X$ и $Y$ - банаховы пространства, то запись $X \subset Y$ всюду в дальнейшем означает, что $X$ линейно и непрерывно вложено в $Y$; тогда, в частности, для некоторой константы $C>0$ и всех $x \in X$ выполнено неравенство $\|x\|_{Y} \leqslant C\left\|_{x}\right\|_{X}$. Не ограничивая общности, будем всегда предполагать, что $\left\|\chi_{[0,1]}\right\|_{X}=1$. Тогда, как нетрудно проверить [16; теорема 2.4.1], для произвольного симметричного пространства $X$ на $[0,1]$ выполнено: $L_{\infty} \subset X \subset L_{1}$, причем $\|x\|_{X} \leqslant\|x\|_{L_{\infty}}$ $\left(x \in L_{\infty}\right)$ и $\|x\|_{L_{1}} \leqslant\|x\|_{X}(x \in X)$. В дальнейшем часто будет использоваться следующее утверждение.

ПрЕДЛОЖЕНИЕ 1 [16; следствие 2.4.2]. Пусть $X$ - симметричное пространство на $J, x \in X, y \in S(J)$. Если для некоторого $C>0$ и всех $\tau>0$ выполнено $n_{|y|}(\tau) \leqslant C n_{|x|}(\tau)$, то $y \in X u\|y\|_{X} \leqslant \max \{1, C\}\|x\|_{X}$.

Если $X$ - симметричное пространство на $J$, то двойственное (или ассоциированное) пространство $X^{\times}$состоит из всех $y \in S(J)$, для которых

$$
\|y\|_{X^{\times}}=\sup \left\{\int_{J} x(t) y(t) d t:\|x\|_{X} \leqslant 1\right\}<\infty .
$$

Пространство $X^{\times}$также симметрично; оно изометрически вложено в сопряженное $X^{*}$; при этом $X^{\times}=X^{*}$ тогда и только тогда, когда $X$ сепарабельно. Любое симметричное пространство $X$ непрерывно вложено в свое второе двойственное, которое будет обозначаться через $X^{\times \times}$. Симметричное пространство $X$ называется максимальным (имеет свойство Фату), если из того, что $x_{n} \in X \quad(n=1,2, \ldots), \sup _{n=1,2, \ldots}\left\|x_{n}\right\|_{X}<\infty, x \in S(J)$ и $x_{n} \rightarrow x$ п. в., следует: $x \in X$ и $\|x\|_{X} \leqslant \liminf _{n \rightarrow \infty}\left\|x_{n}\right\|_{X}$. Если симметричное пространство $X$ обладает аналогичным свойством при дополнительном условии $x \in X$, то говорят, 
что $X$ имеет порядково полунепрерывную норму. Последнее эквивалентно тому, что $X$ вложено в $X^{\times \times}$изометрически [19; теорема 6.1.6]. Пространство $X$ максимально, если и только если его естественное вложение в $X^{\times \times}$является сюръективной изометрией [19; теорема 6.1.7]. Норма любого максимального или сепарабельного симметричного пространства порядково полунепрерывна.

Приведем примеры симметричных пространств. Во-первых, это пространства $L_{p}(1 \leqslant p \leqslant \infty)$ :

$$
\|x\|_{p}= \begin{cases}\left(\int_{0}^{1}|x(t)|^{p} d t\right)^{1 / p}, & 1 \leqslant p<\infty \\ \underset{t \in[0,1]}{\operatorname{ess} \sup }|x(t)|, & p=\infty\end{cases}
$$

а также их обобщение - семейство пространств $L_{p, q}(1<p<\infty, 1 \leqslant q \leqslant \infty)$ :

$$
\|x\|_{p, q}= \begin{cases}{\left[\frac{q}{p} \int_{0}^{1}\left(t^{1 / p} x^{*}(t)\right)^{q} \frac{d t}{t}\right]^{1 / q},} & 1 \leqslant q<\infty, \\ \operatorname{ess\operatorname {sup}}\left(t^{1 / p} x^{*}(t)\right), & q=\infty, \\ t \in[0,1] & \end{cases}
$$

играющих важную роль в теории интерполяции операторов. Хотя функционал $\|x\|_{p, q}$ не субаддитивен, он эквивалентен норме $\|x\|_{p, q}^{\prime}=\left\|x^{* *}\right\|_{p, q}$, где $x^{* *}(t)=\frac{1}{t} \int_{0}^{t} x^{*}(s) d s$. Нетрудно проверить [16; лемма 2.6.5], что $L_{p, q_{1}} \subset L_{p, q_{2}}$ $\left(1 \leqslant q_{1} \leqslant q_{2} \leqslant \infty\right)$ и $L_{p, p}=L_{p}$.

Другим естественным обобщением $L_{p}$-пространств являются пространства Орлича [20]. Если $M(u)$ - функция Орлича, т. е. возрастающая выпуклая функция на $[0, \infty), M(0)=0$, то пространство Орлича $L_{M}$ состоит из всех таких $x \in S(0,1)$, что существует $u>0$, при котором $\int_{0}^{1} M(|x(t)| / u) d t \leqslant 1$. В дальнейшем в качестве нормы в этом пространстве мы будем использовать норму Люксембурга $\|x\|_{L_{M}}=\inf u$, где точная нижняя грань берется по всем $u$, для которых выполнено последнее неравенство. Особый интерес для нас будут представлять так называемые экспоненциальные пространства Орлича. Как легко проверить, каждая из функций

$$
N_{p}(t):=e^{t^{p}}-\sum_{k=0}^{[1 / p]} \frac{t^{k p}}{k !} \quad(0<p<1) \quad \text { и } \quad N_{p}(t):=e^{t^{p}}-1 \quad(p \geqslant 1)
$$

является функцией Орлича на $[0, \infty)$ (всюду далее $[z]$ - целая часть числа $z$ ). Соответствующее пространство Орлича $L_{N_{p}}$ часто обозначается через $\exp L_{p}$. Кроме того, положим: $\exp L_{\infty}:=L_{\infty}$.

Напомним, что неотрицательная функция $\varphi(t)$, определенная на $[0,1]$, называется квазивогнутой, если $\varphi(0)=0, \varphi(t)$ возрастает, а $\varphi(t) / t$ убывает. В частности, всякая неотрицательная возрастающая вогнутая на $[0,1]$ функция $\psi(t)$, $\psi(0)=0$, квазивогнута [16; гл. $2, \S 1$, с. 67]. С другой стороны, всякая квазивогнутая функция $\varphi(t)$ эквивалентна своей наименьшей вогнутой мажоранте $\bar{\varphi}(t)[16 ;$ теорема 2.1.1], точнее, $\bar{\varphi}(t) / 2 \leqslant \varphi(t) \leqslant \bar{\varphi}(t) \quad(0 \leqslant t \leqslant 1)$. 
Важную роль в дальнейшем играет класс $\Psi$, состоящий из всех квазивогнутых на $[0,1]$ функций $\psi(t)$, удовлетворяющих условиям: $\psi(0)=\psi(+0)=0$ и $\psi(1)=1$. Если $\psi \in \Psi$, то пространства Лоренца $\Lambda_{\psi}$ и Марцинкевича $M_{\psi}$ состоят из всех измеримых на $[0,1]$ функций $x(t)$, для которых соответственно

$$
\|x\|_{\Lambda_{\psi}}:=\int_{0}^{1} x^{*}(t) d \bar{\psi}(t)<\infty \quad \text { и } \quad\|x\|_{M_{\psi}}:=\sup _{0<t \leqslant 1} \frac{1}{\psi(t)} \int_{0}^{t} x^{*}(s) d s<\infty .
$$

Через $X_{\circ}$ мы будем обозначать сепарабельную часть симметричного пространства $X$, т. е. замыкание $L_{\infty}$ в $X$. Заметим, что $X_{\circ}$ само является симметричным пространством, которое сепарабельно, если только $X \neq L_{\infty}[16$; лемма 2.4.4]. В частности, пространство $\left(M_{\psi}\right)$ 。 может быть охарактеризовано как множество всех таких функций $x \in M_{\psi}$, что $\lim _{t \rightarrow 0+} \frac{1}{\psi(t)} \int_{0}^{t} x^{*}(s) d s=0$. Нетрудно показать, что для каждого симметричного пространства $X$ имеет место равенство $\left(X^{\times \times}\right)_{\circ}=X_{\circ}$.

Пространство $\Lambda_{\psi}$ сепарабельно и максимально, $\left(M_{\psi}\right)$ 。 сепарабельно, но не максимально, напротив, $M_{\psi}$ максимально, но не сепарабельно. Справедливы также следующие соотношения двойственности: $\left(\Lambda_{\psi}\right)^{\times}=M_{\psi}$ и $\left(M_{\psi}\right)^{\times}=$ $\left(\left(M_{\psi}\right)_{\circ}\right)^{\times}=\Lambda_{\psi}[16 ;$ теоремы 2.5.2 и 2.5.4].

Важной характеристикой симметричного пространства $X$ является его фундаментальная функиия $\phi_{X}(s):=\left\|\chi_{(0, s)}\right\|_{X}(0 \leqslant s \leqslant 1)$. В частности,

$$
\begin{gathered}
\phi_{L_{p, q}}(s)=s^{1 / p} \quad(1 \leqslant q \leqslant \infty), \\
\phi_{\Lambda_{\varphi}}(s)=\bar{\varphi}(s) \quad \text { и } \quad \phi_{M_{\varphi}}(s)=\widetilde{\varphi}(s), \quad \text { где } \widetilde{\varphi}(s)=\frac{s}{\varphi(s)} .
\end{gathered}
$$

Для любого симметричного пространства $X$ функция $\phi_{X}$ квазивогнута на $[0,1]$ (см. [16; теорема 2.4.7]), и если $\phi-$ фундаментальная функция симметричного пространства $X$, то $\Lambda_{\phi} \subset X \subset M_{\tilde{\phi}}$ [16; теоремы 2.5.5 и 2.5.7].

В каждом симметричном пространстве $X$ на $[0,1]$ непрерывен оператор pacтяэсения

$$
\sigma_{\tau} x(t):=x\left(\frac{t}{\tau}\right) \chi_{[0, \min \{1, \tau\}]}(t) \quad(\tau>0)
$$

и $\left\|\sigma_{\tau}\right\|_{X \rightarrow X} \leqslant \max \{1, \tau\}$ [16; теорема 2.4.5]. С его помощью определяются нижний и верхний индексы Бойда пространства $X$ :

$$
\alpha_{X}:=\lim _{\tau \rightarrow 0+} \frac{\ln \left\|\sigma_{\tau}\right\|_{X \rightarrow X}}{\ln \tau} \quad \text { и } \quad \beta_{X}:=\lim _{\tau \rightarrow+\infty} \frac{\ln \left\|\sigma_{\tau}\right\|_{X \rightarrow X}}{\ln \tau} .
$$

Из приведенной оценки нормы оператора растяжения следует, что всегда выполнены неравенства $0 \leqslant \alpha_{X} \leqslant \beta_{X} \leqslant 1$. В частности, $\alpha_{L_{p, q}}=\beta_{L_{p, q}}=1 / p$, а индексы Бойда пространств Лоренца и Марцинкевича совпадают с соответствующими показателями растяжения их фундаментальной функции [16; гл. 2 , $\S 4$, c. 138$]$.

Далее нам понадобится понятие симметричного пространства случайных величин, определенных на вероятностном пространстве $(\Omega, \Sigma, \mathrm{P})$. А именно, если $X$ - симметричное пространство на $[0,1]$, то соответствующее пространство 
$X(\Omega):=X(\Omega, \Sigma, \mathrm{P})$ состоит из таких с. в. $f: \Omega \rightarrow \mathbb{R}$, что $f^{*} \in X$, и $\|f\|_{X(\Omega)}:=$ $\left\|f^{*}\right\|_{X}$. Здесь, как и ранее,

$$
f^{*}(t)=\inf \{\tau \geqslant 0: \mathrm{P}\{\omega \in \Omega:|f(\omega)|>\tau\}<t\} \quad(0<t \leqslant 1) .
$$

Если пространство $(\Omega, \Sigma, \mathrm{P})$ изоморфно отрезку $[0,1]$, рассматриваемому с мерой Лебега, то симметричные пространства $X(\Omega)$ и $X$ мы можем (и будем) отождествлять.

Важную роль в дальнейшем будут играть понятия и методы теории интерполяции операторов. Совокупность $\vec{X}=\left(X_{0}, X_{1}\right)$ двух банаховых пространств $X_{0}$ и $X_{1}$ называют банаховой парой, если они оба линейно и непрерывно вложены в одно и то же отделимое линейное топологическое пространство. Для банаховой пары $\left(X_{0}, X_{1}\right)$ можно определить пересечение $X_{0} \cap X_{1}$ и сумму $X_{0}+X_{1}$ как банаховы пространства с нормами

$$
\|x\|_{X_{0} \cap X_{1}}=\max \left\{\|x\|_{X_{0}},\|x\|_{X_{1}}\right\}
$$

И

$$
\|x\|_{X_{0}+X_{1}}=\inf \left\{\left\|x_{0}\right\|_{X_{0}}+\left\|x_{1}\right\|_{X_{1}}: x=x_{0}+x_{1}, x_{i} \in X_{i}, i=0,1\right\}
$$

соответственно. Банахову пару образуют, например, любые два симметричных пространства $X_{0}$ и $X_{1}$ на $J$, где $J=[0,1]$ или $J=(0, \infty)$, так как $X_{i} \subset S(J)$ $(i=0,1)$.

Говорят, что банахово пространство $X$ интерполяционно относительно банаховой пары $\left(X_{0}, X_{1}\right)\left(X \in \operatorname{Int}\left(X_{0}, X_{1}\right)\right)$, если $X_{0} \cap X_{1} \subset X \subset X_{0}+X_{1}$ и для любого линейного оператора $T$, ограниченного в $X_{0}$ и в $X_{1}$, имеем: $T: X \rightarrow X$. В этом случае по теореме о замкнутом графике найдется такое $C>0$, что для любого $T: X_{i} \rightarrow X_{i}(i=0,1)$ выполнено: $\|T\|_{X \rightarrow X} \leqslant C \max _{i=0,1}\|T\|_{X_{i} \rightarrow X_{i}}$. Если в последнем неравенстве можно взять $C=1$, то пространство $X$ будем называть интерполяиионным с константой 1 . В частности, согласно классической теореме Рисса-Торина [21; теорема 1.1.1] пространство $L_{p}$ интерполяционно с константой 1 относительно пары $\left(L_{p_{0}}, L_{p_{1}}\right)$ для любых $1 \leqslant p_{0} \leqslant p \leqslant p_{1} \leqslant \infty$.

Один из наиболее важных и в теории, и в приложениях способ построения интерполяционных пространств связан с использованием $\mathscr{K}$-функционала Петре, определенного на банаховой паре $\left(X_{0}, X_{1}\right)$ следующим образом:

$$
\mathscr{K}\left(t, x ; X_{0}, X_{1}\right)=\inf \left\{\left\|x_{0}\right\|_{X_{0}}+t\left\|x_{1}\right\|_{X_{1}}: x=x_{0}+x_{1}, x_{i} \in X_{i}\right\},
$$

где $x \in X_{0}+X_{1}$, а $t>0$. Нетрудно показать, что если $\left(X_{0}, X_{1}\right)$ - банахова пара и $x \in X_{0}+X_{1}$ фиксировано, то $\mathscr{K}\left(t, x ; X_{0}, X_{1}\right)$ - непрерывная неотрицательная возрастающая вогнутая функция относительно переменной $t>0$ [21; лемма 3.1.1]. Если $X_{0} \subset X_{1}$ и $\|x\|_{X_{1}} \leqslant\|x\|_{X_{0}}\left(x \in X_{0}\right)$ (соответственно $X_{1} \subset X_{0}$ и $\left.\|x\|_{X_{0}} \leqslant\|x\|_{X_{1}}\left(x \in X_{1}\right)\right)$, то $\mathscr{K}\left(t, x ; X_{0}, X_{1}\right)=t\|x\|_{X_{1}}$ при $0 \leqslant t \leqslant 1$ (соответственно $\mathscr{K}\left(t, x ; X_{0}, X_{1}\right)=\|x\|_{X_{0}}$ при $\left.t \geqslant 1\right)$.

Две неотрицательные функции $f(t)$ и $g(t)$ далее будем называть эквивалентными на множестве $T$ (пишем: $f \asymp g(t \in T)$ ), если для некоторой константы $C>0$ и всех $t \in T$ выполнено: $C^{-1} f(t) \leqslant g(t) \leqslant C f(t)$. 
В ряде случаев для $\mathscr{K}$-функционала удается найти достаточно простое эквивалентное выражение. В частности, для произвольного пространства с $\sigma$-конечной мерой и любого $p \geqslant 1$

$$
\mathscr{K}\left(t, x ; L_{p}, L_{\infty}\right) \asymp\left(\int_{0}^{t^{p}}\left(x^{*}(s)\right)^{p} d s\right)^{1 / p} \quad(t>0),
$$

где константы эквивалентности не зависят от $x \in L_{p}+L_{\infty}$ и $t>0$ (при $p=1$ эквивалентность можно заменить равенством).

Далее, если $F$ - банахова решетка двусторонних числовых последовательностей, то через $F\left(u_{k}\right), u_{k}>0(k=0, \pm 1, \pm 2, \ldots)$, будем обозначать весовое пространство, состоящее из всех последовательностей $a=\left(a_{k}\right)_{k=-\infty}^{\infty}$, для которых конечна норма $\|a\|_{F\left(u_{k}\right)}=\left\|\left(a_{k} u_{k}\right)\right\|_{F}$. Предположим, что $F \supset$ $\ell_{\infty} \cap \ell_{\infty}\left(2^{-k}\right)$. Если $\left(X_{0}, X_{1}\right)$ - произвольная банахова пара, то пространство вещественного $\mathscr{K}$-метода $\left(X_{0}, X_{1}\right)_{F}^{\mathscr{K}}$ состоит из всех таких $x \in X_{0}+X_{1}$, что $\left(\mathscr{K}\left(2^{k}, x ; X_{0}, X_{1}\right)\right)_{k} \in F$, с нормой $\|x\|=\left\|\left(\mathscr{K}\left(2^{k}, x ; X_{0}, X_{1}\right)\right)_{k}\right\|_{F}$. Нетрудно проверить, что $\left(X_{0}, X_{1}\right)_{F}^{\mathscr{K}}$ интерполяционно относительно пары $\left(X_{0}, X_{1}\right)$ с константой 1 [22]. В частном случае $F=\ell_{p}\left(2^{-k \theta}\right)(0<\theta<1,1 \leqslant p \leqslant \infty)$ получаем классические пространства $\left(X_{0}, X_{1}\right)_{\theta, p}$, свойства которых подробно изложены в монографии [21].

Важность вещественного метода интерполяции связана, в частности, с тем, что для достаточно обширного класса банаховых пар за счет него получаются все интерполяционные пространства. Банахова пара $\vec{X}=\left(X_{0}, X_{1}\right)$ называется $\mathscr{K}$-монотонной (или парой Калъдерона-Митягина), если выполнение неравенства

$$
\mathscr{K}\left(t, y ; X_{0}, X_{1}\right) \leqslant \mathscr{K}\left(t, x ; X_{0}, X_{1}\right) \quad(t>0)
$$

для некоторых $x \in X_{0}+X_{1}$ и $y \in X_{0}+X_{1}$ влечет существование оператора $U$, ограниченного в $X_{0}$ и в $X_{1}$, такого, что $y=U x$. Ввиду теоремы Брудного-Кругляка, одного из центральных результатов в теории интерполяции $[22$; теорема 15.1], любое пространство $X$, интерполяционное относительно $\mathscr{K}$-монотонной пары $\left(X_{0}, X_{1}\right)$, представимо в виде $X=\left(X_{0}, X_{1}\right)_{F}^{\mathscr{K}}$ для некоторой банаховой решетки $F$.

Второе название $\mathscr{K}$-монотонной пары объясняется тем, что исторически первым результатом в этом направлении явилась теорема о $\mathscr{K}$-монотонности банаховой пары $\left(L_{1}, L_{\infty}\right)$, доказанная независимо и почти одновременно А. П. Кальдероном [23] и Б. С. Митягиным [24]. В дальнейшем мы всегда будем предполагать, что симметричное пространство $X$ на $[0,1]$ интерполяционно относительно этой пары с константой 1. Ввиду теоремы Кальдерона-Митягина это эквивалентно выполнению следующего условия: если $x \in X, y \in L_{1}$ и $y \prec x$, то $y \in X$ и $\|y\|_{X} \leqslant\|x\|_{X}$, где через $y \prec x$ обозначается полуупорядоченность Харди-Литтлвуда, означающая, что $\int_{0}^{t} y^{*}(s) d s \leqslant \int_{0}^{t} x^{*}(s) d s$ при всех $0 \leqslant t \leqslant 1$. Класс таких пространств весьма широк, он содержит все максимальные и сепарабельные пространства, в частности, все пространства Орлича, Лоренца и Марцинкевича. Ввиду [17; теорема 2.а.4] оператор условного математического ожидания $\mathrm{E}_{\mathscr{B}}$, соответствующий $\sigma$-подалгебре $\mathscr{B}$ некоторого 
вероятностного пространства, ограничен в $L_{1}$ и в $L_{\infty}$ с константой 1 . Поэтому справедливо следующее утверждение, которое далее будем часто использовать.

ПрЕДЛОЖЕНИЕ 2. Всякий оператор условного математического ожидания $\mathrm{E}_{\mathscr{B}}$ ограничен в любом симметричном пространстве и имеет норму 1.

Наконец, для нас будет полезна следующая интерполяционная конструкция, введенная А. П. Кальдероном [25]. Пусть $X_{0}$ и $X_{1}$ - банаховы решетки измеримых функций, определенных на одном и том же пространстве с мерой $(\mathscr{M}, m)$. Для каждого $\theta \in(0,1)$ пространство $X_{0}^{1-\theta} X_{1}^{\theta}$ состоит из всех таких измеримых функций $f$ на $(\mathscr{M}, m)$, что при некоторых $\lambda>0$ и $f_{i} \in X_{i},\left\|f_{i}\right\|_{X_{i}} \leqslant 1(i=0,1)$, справедливо неравенство

$$
|f(x)| \leqslant \lambda\left|f_{0}(x)\right|^{1-\theta}\left|f_{1}(x)\right|^{\theta}, \quad x \in \mathscr{M} .
$$

Это пространство снабжается нормой, равной точной нижней грани чисел $\lambda$, для которых выполнено предыдущее соотношение. Несмотря на то что эта конструкция не является интерполяционным функтором на множестве всех пар банаховых решеток [26], она очень полезна в теории интерполяции. В частности, если $\left(X_{0}, X_{1}\right)$ и $\left(Y_{0}, Y_{1}\right)$ - две пары банаховых решеток измеримых функций на пространствах с мерой $(\mathscr{M}, m)$ и $\left(\mathscr{M}^{\prime}, m^{\prime}\right)$ соответственно, то любой положительный оператор $A$ из $S(\mathscr{M}, m)$ в $S\left(\mathscr{M}^{\prime}, m^{\prime}\right)$, который ограничен из $X_{0}$ в $Y_{0}$ и из $X_{1}$ в $Y_{1}$, ограничен также из пространства $X_{0}^{1-\theta} X_{1}^{\theta}$ в пространство $Y_{0}^{1-\theta} Y_{1}^{\theta}$ и $\|A\|_{X_{0}^{1-\theta} X_{1}^{\theta} \rightarrow Y_{0}^{1-\theta} Y_{1}^{\theta}} \leqslant\|A\|_{X_{0} \rightarrow Y_{0}}^{1-\theta}\|A\|_{X_{1} \rightarrow Y_{1}}^{\theta}$ для всех $\theta \in(0,1)$ (см., например, [17; предложение 1.d.2(i), с. 43]).

Детальное изложение как приведенных здесь, так и многих других результатов и фактов теории интерполяции можно найти в монографиях [16], [18], [21], [22].

В разделах 6 и 7 мы будем говорить о (квази)банаховых симметричных пространствах последовательностей. Если $\xi=\left(\xi_{n}\right)_{n=1}^{\infty}-$ ограниченная последовательность вещественных чисел, то $\xi^{*}=\left(\xi_{n}^{*}\right)_{n=1}^{\infty}-$ ее невозрастающая перестановка, т. е. $\xi_{n}^{*}=\inf _{\text {card } A=n-1} \sup _{k \in \mathbb{N} \backslash A}\left|\xi_{k}\right|$. Квазибанахово пространство последовательностей $E$ называется симметричным, если из условий $a \in E$ и $b^{*} \leqslant a^{*}$ следует, что $b \in E$ и $\|b\|_{E} \leqslant\|a\|_{E}$. В таком пространстве квазинорма $\|\cdot\|_{E}$ удовлетворяет обобщенному неравенству треугольника

$$
\|a+b\|_{E} \leqslant L\left(\|a\|_{E}+\|b\|_{E}\right), \quad a, b \in E,
$$

где $L \geqslant 1$. Если последнее неравенство выполнено в случае $L=1$, то $E$ называется (банаховым) симметричным пространством последовательностей. Без ограничения общности всюду будем предполагать, что $\left\|e_{k}\right\|_{E}=1(k=1,2, \ldots)$, где $e_{k}$ - элементы стандартного базиса в пространствах последовательностей.

\section{3. Оценки $L_{p}$-норм сумм независимых функций}

В этом разделе мы рассмотрим оценки $L_{p}$-норм сумм независимых функций, обобщающие и уточняющие классические неравенства Хинчина и Марцинкевича-Зигмунда. Здесь можно выделить два основных направления. Первое 
связано с тем, что вместо равномерно ограниченных рассматриваются произвольные системы неотрицательных или симметрично распределенных функций. Суть второго состоит в замене нормы последовательности коэффициентов в пространстве $\ell_{2}$ (или более общо: суммы дисперсий слагаемых) некоторой более "гибкой" величиной, используя которую, можно получить двусторонние оценки с константой, не зависящей от $p$.

3.1. Неравенства Розенталя. В 1970 г. при изучении дополняемых подпространств пространства $L_{p}=L_{p}[0,1]$ Х. П. Розенталь [1] доказал следующие замечательные соотношения, второе из которых можно рассматривать как далеко идущее обобщение неравенств Хинчина. Они показывают, что с точностью до эквивалентности $L_{p}$-норма суммы независимых функций определяется $L_{q}$-нормами слагаемых. Как мы увидим далее, аналогичное явление, выраженное в терминах “дизъюнктификации”, наблюдается также и в общих симметричных пространствах.

Теорема 6 (неравенства Розенталя).

(i) Если $1<p<\infty$, то для произвольной последовательности $\left\{f_{k}\right\}_{k=1}^{\infty} \subset L_{p}$ неотрицательных независимых функиий и каждого $n \in \mathbb{N}$ справедливо неравенство

$$
\begin{aligned}
& \max \left\{\left(\sum_{k=1}^{n}\left\|f_{k}\right\|_{p}^{p}\right)^{1 / p}, \sum_{k=1}^{n}\left\|f_{k}\right\|_{1}\right\} \leqslant\left\|\sum_{k=1}^{n} f_{k}\right\|_{p} \\
& \leqslant 2^{p} \max \left\{\left(\sum_{k=1}^{n}\left\|f_{k}\right\|_{p}^{p}\right)^{1 / p}, \sum_{k=1}^{n}\left\|f_{k}\right\|_{1}\right\} .
\end{aligned}
$$

(ii) Если $p>2$, то существует такая константа $K_{p}>0$, что для произвольной последовательности $\left\{f_{k}\right\}_{k=1}^{\infty} \subset L_{p}$ независимых функиий со свойством $\int_{0}^{1} f_{k}(t) d t=0 \quad(k=1,2, \ldots)$ и каждого $n \in \mathbb{N}$ выполнено:

$$
\begin{aligned}
\frac{1}{2} \max \left\{\left(\sum_{k=1}^{n}\left\|f_{k}\right\|_{p}^{p}\right)^{1 / p}\right. & \left.,\left(\sum_{k=1}^{n}\left\|f_{k}\right\|_{2}^{2}\right)^{1 / 2}\right\} \leqslant\left\|\sum_{k=1}^{n} f_{k}\right\|_{p} \\
& \leqslant K_{p} \max \left\{\left(\sum_{k=1}^{n}\left\|f_{k}\right\|_{p}^{p}\right)^{1 / p},\left(\sum_{k=1}^{n}\left\|f_{k}\right\|_{2}^{2}\right)^{1 / 2}\right\} .
\end{aligned}
$$

Для доказательства нам потребуются две леммы.

Лемма 1. Пусть $1<p<\infty$. Если $\left\{f_{k}\right\}_{k=1}^{\infty} \subset L_{p}$ - произвольная последовательность независимых функиий, то для каждого $n \in \mathbb{N}$

$$
\left\|\sum_{k=1}^{n} f_{k}\right\|_{p} \leqslant 2^{p} \max \left\{\left(\sum_{k=1}^{n}\left\|f_{k}\right\|_{p}^{p}\right)^{1 / p}, \sum_{k=1}^{n}\left\|f_{k}\right\|_{1}\right\} .
$$

ДокАЗАТЕЛЬСтво. Мы можем (и будем) предполагать, что $f_{k} \geqslant 0$ для всех $k=1,2, \ldots$. Введем обозначение:

$$
N_{p}:=\left(\sum_{k=1}^{n}\left\|f_{k}\right\|_{p}^{p}\right)^{1 / p} .
$$


Ввиду независимости функций $f_{1}$ и $f_{2}+\cdots+f_{n}$

$$
\begin{aligned}
\int_{0}^{1}\left(f_{1}+\cdots+f_{n}\right)^{p-1} f_{1} d t & \leqslant 2^{p-1} \int_{0}^{1}\left(f_{1}^{p-1}+\left(f_{2}+\cdots+f_{n}\right)^{p-1}\right) f_{1} d t \\
& =2^{p-1}\left(\int_{0}^{1} f_{1}^{p} d t+\int_{0}^{1}\left(f_{2}+\cdots+f_{n}\right)^{p-1} d t \int_{0}^{1} f_{1} d t\right) \\
& \leqslant 2^{p-1}\left(\int_{0}^{1} f_{1}^{p} d t+\int_{0}^{1}\left(f_{1}+\cdots+f_{n}\right)^{p-1} d t \int_{0}^{1} f_{1} d t\right) .
\end{aligned}
$$

Точно так же

$$
\int_{0}^{1}\left(f_{1}+\cdots+f_{n}\right)^{p-1} f_{k} d t \leqslant 2^{p-1}\left(\int_{0}^{1} f_{k}^{p} d t+\left\|\sum_{i=1}^{n} f_{i}\right\|_{p-1}^{p-1} \int_{0}^{1} f_{k} d t\right)
$$

для всех $k=1, \ldots, n$. Суммируя последние неравенства по $k$, получим

$$
\left\|\sum_{k=1}^{n} f_{k}\right\|_{p}^{p} \leqslant 2^{p-1}\left(N_{p}^{p}+\left\|\sum_{k=1}^{n} f_{k}\right\|_{p}^{p-1} N_{1}\right) \leqslant 2^{p} \max \left\{N_{p}^{p},\left\|\sum_{k=1}^{n} f_{k}\right\|_{p}^{p-1} N_{1}\right\},
$$

откуда

$$
\left\|\sum_{k=1}^{n} f_{k}\right\|_{p} \leqslant \max \left\{2 N_{p}, 2^{p} N_{1}\right\} \leqslant 2^{p} \max \left\{N_{p}, N_{1}\right\} .
$$

Лемма доказана.

Лемма 2. Пусть $1 \leqslant p<\infty u\left\{f_{k}\right\}_{k=1}^{\infty} \subset L_{p}-$ произвольная последовательность независимых функиий такая, что $\int_{0}^{1} f_{k}(t) d t=0 \quad(k=1,2, \ldots)$. Тогда:

(а) если $\theta_{k}= \pm 1 \quad(k=1,2, \ldots)$, то для каждого $n \in \mathbb{N}$

$$
\left\|\sum_{k=1}^{n} \theta_{k} f_{k}\right\|_{p} \leqslant 2\left\|\sum_{k=1}^{n} f_{k}\right\|_{p}
$$

(b) длякаждого $n \in \mathbb{N}$

$$
\left\|\sum_{k=1}^{n} f_{k}\right\|_{p} \leqslant 2\left(\sum_{k=1}^{n}\left\|f_{k}\right\|_{p}^{p}\right)^{1 / p}, \quad \text { если } p \leqslant 2
$$

$u$

$$
\left\|\sum_{k=1}^{n} f_{k}\right\|_{p} \geqslant \frac{1}{2}\left(\sum_{k=1}^{n}\left\|f_{k}\right\|_{p}^{p}\right)^{1 / p}, \quad \text { если } p>2 .
$$

ДокАзАтЕльство. Предположим, что функции $f$ и $g$ независимы и, кроме того, $\mathrm{E} f=0$. Тогда, если $\mathrm{E}_{g}$ - оператор условного математического ожидания относительно $\sigma$-алгебры, порожденной функцией $g$, то выполнены неравенства $\left\|\mathrm{E}_{g}\right\|_{L_{p} \rightarrow L_{p}} \leqslant 1 \quad(1 \leqslant p \leqslant \infty)$ (см. предложение 2$)$. Кроме того, так как для 
любого $g$-измеримого множества $B$ функции $f$ и $\chi_{B}$ независимы, то ввиду равенства (5)

$$
\int_{B} \mathrm{E}_{g} f d u=\int_{B} f d u=\int_{0}^{1} f \chi_{B} d u=\mathrm{E} f \cdot \lambda(B)=0 .
$$

Поэтому $\mathrm{E}_{g} f=0$ и

$$
\|g\|_{p}=\left\|\mathrm{E}_{g} g\right\|_{p}=\left\|\mathrm{E}_{g}(f+g)\right\|_{p} \leqslant\|f+g\|_{p} .
$$

Для данных $\theta_{k}= \pm 1(k=1, \ldots, n)$ определим

$$
f=\sum_{k: \theta_{k}=1} f_{k} \quad \text { и } \quad g=\sum_{k: \theta_{k}=-1} f_{k} .
$$

Тогда

$$
f+g=\sum_{k=1}^{n} f_{k} \quad \text { и } \quad f-g=\sum_{k=1}^{n} \theta_{k} f_{k}
$$

По условию $f$ и $g$ независимы и $\int_{0}^{1} f(t) d t=\int_{0}^{1} g(t) d t=0$. Следовательно, ввиду сделанного ранее наблюдения, $\|f\|_{p} \leqslant\|f+g\|_{p}$ и $\|g\|_{p} \leqslant\|f+g\|_{p}$. Тем самым, $\|f-g\|_{p} \leqslant 2\|f+g\|_{p}$, и (а) доказано.

Переходя к доказательству утверждения (b), предположим сначала, что функции $f_{k}(k=1,2, \ldots)$ симметрично распределены. Тогда функции $f_{1}-f_{2}$ и $f_{1}+f_{2}$ одинаково распределены и, значит, $\left\|f_{1}+f_{2}\right\|_{p}^{p}=\left(\left\|f_{1}+f_{2}\right\|_{p}^{p}+\right.$ $\left.\left\|f_{1}-f_{2}\right\|_{p}^{p}\right) / 2$. Если $1 \leqslant p \leqslant 2$, то $|a+b|^{p}+|a-b|^{p} \leqslant 2\left(|a|^{p}+|b|^{p}\right)$, и поэтому

$$
\left\|f_{1}+f_{2}\right\|_{p}^{p} \leqslant\left\|f_{1}\right\|_{p}^{p}+\left\|f_{2}\right\|_{p}^{p} .
$$

В случае, когда $p>2$, справедливы противоположные неравенства:

$$
|a+b|^{p}+|a-b|^{p} \geqslant 2\left(|a|^{p}+|b|^{p}\right) \quad \text { и } \quad\left\|f_{1}+f_{2}\right\|_{p}^{p} \geqslant\left\|f_{1}\right\|_{p}^{p}+\left\|f_{2}\right\|_{p}^{p} .
$$

Тем самым, если функции $f_{k}$ независимы и симметрично распределены, то для каждого $n \in \mathbb{N}$

$$
\left\|\sum_{k=1}^{n} f_{k}\right\|_{p} \leqslant\left(\sum_{k=1}^{n}\left\|f_{k}\right\|_{p}^{p}\right)^{1 / p}, \quad \text { если } p \leqslant 2
$$

и

$$
\left\|\sum_{k=1}^{n} f_{k}\right\|_{p} \geqslant\left(\sum_{k=1}^{n}\left\|f_{k}\right\|_{p}^{p}\right)^{1 / p}, \quad \text { если } p>2 .
$$

В общем случае, применяя симметризацию, рассмотрим разности $f_{i}-f_{i}^{\prime}$, где $f_{i}^{\prime}$ - независимые копии $f_{i}(i=1,2, \ldots)$. Они независимы и симметрично распределены. Поэтому, если, например, $1 \leqslant p \leqslant 2$, то с помощью рассуждений из начала доказательства, а также неравенства Минковского получаем:

$$
\left\|\sum_{k=1}^{n} f_{k}\right\|_{p} \leqslant\left\|\sum_{k=1}^{n}\left(f_{k}-f_{k}^{\prime}\right)\right\|_{p} \leqslant\left(\sum_{k=1}^{n}\left\|f_{k}-f_{k}^{\prime}\right\|_{p}^{p}\right)^{1 / p} \leqslant 2\left(\sum_{k=1}^{n}\left\|f_{k}\right\|_{p}^{p}\right)^{1 / p} .
$$

Случай $p>2$ рассматривается аналогично. Лемма доказана. 
ДоКАЗАТЕЛЬСТво тЕОРЕМЫ 6. Чтобы получить утверждение (i), достаточно применить лемму 1 , а также элементарное неравенство $\left\|\left(b_{k}\right)\right\|_{p} \leqslant\left\|\left(b_{k}\right)\right\|_{1}$ $\left(p \geqslant 1, b_{k} \in \mathbb{R}\right)$.

При доказательстве (ii) будет использоваться обозначение, введенное в доказательстве леммы 1. Так как $f_{k}$ независимы, то $\int_{0}^{1} f_{i} f_{j} d t=\int_{0}^{1} f_{i} d t \int_{0}^{1} f_{j} d t=0$ $(i \neq j)$, т. е. система $\left\{f_{k}\right\}_{k=1}^{\infty}$ ортогональна. Поэтому

$$
N_{2}=\left\|\sum_{k=1}^{n} f_{k}\right\|_{2}
$$

и тогда оценка снизу в (7) следует из леммы 2 (b), а также того, что

$$
\left\|\sum_{k=1}^{n} f_{k}\right\|_{2} \leqslant\left\|\sum_{k=1}^{n} f_{k}\right\|_{p}
$$

Докажем оценку сверху в (7). По лемме 2 (а) для каждого $u \in[0,1]$ мы имеем

$$
\left\|\sum_{k=1}^{n} f_{k}\right\|_{p}^{p} \leqslant 2^{p} \int_{0}^{1}\left|\sum_{k=1}^{n} r_{k}(u) f_{k}(t)\right|^{p} d t
$$

где $r_{k}(u)$ - функции Радемахера. Интегрируя последнее неравенство по $u$, меняя порядок интегрирования и применяя неравенства Хинчина (1), получим

$$
\left\|\sum_{k=1}^{n} f_{k}\right\|_{p}^{p} \leqslant 2^{p} B_{p}^{p} \int_{0}^{1}\left(\sum_{k=1}^{n} f_{k}(t)^{2}\right)^{p / 2} d t .
$$

По условию $f_{k}^{2}-$ неотрицательные независимые функции из $L_{p / 2}$. Так как $p / 2>1$, то по лемме 1

$$
\left(\int_{0}^{1}\left(\sum_{k=1}^{n} f_{k}(t)^{2}\right)^{p / 2} d t\right)^{2 / p} \leqslant 2^{p / 2} \max \left\{N_{p}^{2}, N_{2}^{2}\right\} .
$$

В итоге оценка сверху в $(7)$ с $K_{p}=2^{p / 4+1} B_{p}$ вытекает из двух последних соотношений. Теорема доказана.

3.2. Неравенства Хитченко. Неравенства Хинчина (1) для $L_{p}$-норм полиномов Радемахера содержат константы $A_{p}$ и $B_{p}$, зависящие от $p$, и поэтому, скажем, на основе правой оценки можно лишь утверждать, что с некоторой константой $C=C(a)$

$$
\left\|\sum_{k=1}^{\infty} a_{k} r_{k}\right\|_{p} \leqslant C \sqrt{p} \quad(p \geqslant 1), \quad \text { если } a=\left(a_{k}\right) \in \ell_{2} .
$$

В 1993 г. П. Хитченко доказал более точное утверждение, которое позволяет в принципе для каждой последовательности коэффициентов $\left(a_{k}\right)_{k=1}^{\infty} \in \ell_{2}$ определить порядок нормы $\left\|\sum_{k=1}^{\infty} a_{k} r_{k}\right\|_{p}$ при $p \rightarrow \infty$ [27]. Центральная роль при этом принадлежит $\mathscr{K}$-функционалу Петре $\kappa_{a}(t):=\mathscr{K}\left(t, a ; \ell_{1}, \ell_{2}\right)$, соответствующему банаховой паре $\left(\ell_{1}, \ell_{2}\right)$ (см. раздел 2$)$. 
Теорема 7. Существует такая универсальная константа $\gamma>0$, что для всех $t \geqslant 1$ и $а=\left(a_{k}\right)_{k=1}^{\infty} \in \ell_{2}$ выполнены неравенства

$$
\gamma \kappa_{a}(\sqrt{t}) \leqslant\left\|\sum_{k=1}^{\infty} a_{k} r_{k}\right\|_{t} \leqslant \kappa_{a}(\sqrt{t}) .
$$

Для доказательства нам потребуются некоторые эквивалентные выражения для функционала $\kappa_{a}(t)$. Прежде всего, мы докажем один из вариантов известного неравенства Хольмстедта (см. [28] или [21; упражнение 5.7.3]).

ПРеДЛОЖеНИЕ 3. Для любых $a=\left(a_{k}\right)_{k=1}^{\infty} \in \ell_{2} u t>0$ выполнены неравенства

$$
\frac{1}{4}\left\{\sum_{i=1}^{\left[t^{2}\right]} a_{i}^{*}+t\left(\sum_{i=\left[t^{2}\right]+1}^{\infty}\left(a_{i}^{*}\right)^{2}\right)^{1 / 2}\right\} \leqslant \kappa_{a}(t) \leqslant \sum_{i=1}^{\left[t^{2}\right]} a_{i}^{*}+t\left(\sum_{i=\left[t^{2}\right]+1}^{\infty}\left(a_{i}^{*}\right)^{2}\right)^{1 / 2}
$$

где $\left(a_{i}^{*}\right)_{i=1}^{\infty}-$ невозрастающая перестановка последователъности $\left(\left|a_{n}\right|\right)_{n=1}^{\infty}$.

ДокАзАТЕльство. Докажем сначала функциональный аналог (9): для произвольной функции $f \in L_{1}(0, \infty)+L_{2}(0, \infty)$ и любых $t>0$

$$
\begin{aligned}
\frac{1}{4}\left\{\int_{0}^{t^{2}} f^{*}(s) d s+t\left(\int_{t^{2}}^{\infty} f^{*}(s)^{2} d s\right)^{1 / 2}\right\} & \leqslant \mathscr{K}\left(t, f ; L_{1}, L_{2}\right) \\
& \leqslant \int_{0}^{t^{2}} f^{*}(s) d s+t\left(\int_{t^{2}}^{\infty} f^{*}(s)^{2} d s\right)^{1 / 2} .
\end{aligned}
$$

Не ограничивая общности, можно считать, что $f=f^{*}$. Для каждого $t>0$ определим $f_{0}=f \chi_{\left(0, t^{2}\right)}$ и $f_{1}=f \chi_{\left(t^{2}, \infty\right)}$. Так как

$$
\left\|f_{0}\right\|_{L_{1}}+t\left\|f_{1}\right\|_{L_{2}}=\int_{0}^{t^{2}} f^{*}(s) d s+t\left(\int_{t^{2}}^{\infty} f^{*}(s)^{2} d s\right)^{1 / 2}
$$

то правое неравенство в (10) вытекает из определения $\mathscr{K}$-функционала.

Для доказательства левого неравенства рассмотрим произвольное представление $f=g+h$, где $g \in L_{1}, h \in L_{2}$. Тогда, ввиду элементарного неравенства для перестановок [16; соотношение (2.23) на с. 93],

$$
f(u) \leqslant g^{*}\left(\frac{u}{2}\right)+h^{*}\left(\frac{u}{2}\right), \quad u>0 .
$$

Поэтому, применяя неравенство Коши-Буняковского, получим

$$
\begin{aligned}
\|g\|_{L_{1}}+t\|h\|_{L_{2}} & \geqslant \frac{1}{2}\left(\int_{0}^{t^{2}} g^{*}\left(\frac{u}{2}\right) d u+t\left(\int_{0}^{t^{2}}\left(h^{*}\left(\frac{u}{2}\right)\right)^{2} d u\right)^{1 / 2}\right) \\
& \geqslant \frac{1}{2}\left(\int_{0}^{t^{2}} g^{*}\left(\frac{u}{2}\right) d u+\int_{0}^{t^{2}} h^{*}\left(\frac{u}{2}\right) d u\right) \geqslant \frac{1}{2} \int_{0}^{t^{2}} f^{*}(u) d u .
\end{aligned}
$$


Кроме того,

$$
\begin{aligned}
t^{2} \int_{t^{2}}^{\infty}\left(g^{*}\left(\frac{u}{2}\right)\right)^{2} d u & \leqslant \int_{0}^{t^{2}} g^{*}\left(\frac{u}{2}\right) d u \int_{t^{2}}^{\infty} g^{*}\left(\frac{u}{2}\right) d u \\
& \leqslant\left(\int_{0}^{\infty} g^{*}\left(\frac{u}{2}\right) d u\right)^{2}=4\|g\|_{L_{1}}^{2}
\end{aligned}
$$

и, значит, ввиду (11)

$$
\begin{aligned}
\|g\|_{L_{1}}+t\|h\|_{L_{2}} & \geqslant \frac{t}{2}\left(\int_{t^{2}}^{\infty}\left(g^{*}\left(\frac{u}{2}\right)\right)^{2} d u\right)^{1 / 2}+\frac{t}{2}\left(\int_{t^{2}}^{\infty}\left(h^{*}\left(\frac{u}{2}\right)\right)^{2} d u\right)^{1 / 2} \\
& \geqslant \frac{t}{2}\left(\int_{t^{2}}^{\infty}\left(f^{*}(u)\right)^{2} d u\right)^{1 / 2} .
\end{aligned}
$$

Отсюда и из (12) по определению $\mathscr{K}$-функционала получаем левое неравенство в (10).

Далее, для произвольной последовательности $a=\left(a_{k}\right)_{k=1}^{\infty} \in \ell_{2}$ определим ступенчатую функцию

$$
f_{a}(t)=\sum_{k=1}^{\infty} a_{k} \chi_{(k-1, k]}(t) \quad(t>0) .
$$

Стандартные рассуждения показывают, что для любых $a=\left(a_{k}\right)_{k=1}^{\infty} \in \ell_{2}$ и $t>0$ справедливо равенство

$$
\mathscr{K}\left(t, f_{a} ; L_{1}, L_{2}\right)=\kappa_{a}(t) .
$$

Перейдем теперь непосредственно к доказательству неравенства (9). Прежде всего, если $0<t<1$, то ввиду неравенства $\|a\|_{2} \leqslant\|a\|_{1}$ имеем: $\kappa_{a}(t)=t\|a\|_{2}$, и (9) очевидно.

Пусть $t \geqslant 1$. Так как пространства $\ell_{1}$ и $\ell_{2}$ симметричны, то можно считать, что $a_{k}=a_{k}^{*}$. Обозначим $b^{t}=\left(b_{k}^{t}\right)$, где $b_{k}^{t}=a_{k}$, если $k \leqslant\left[t^{2}\right]$, и $b_{k}^{t}=0$, если $k>\left[t^{2}\right]$. Кроме того, пусть $c^{t}=a-b^{t}$. Тогда $\kappa_{a}(t) \leqslant\left\|b^{t}\right\|_{1}+t\left\|c^{t}\right\|_{2}$, и тем самым правое неравенство в (9) - прямое следствие определения $\mathscr{K}$-функционала. Что же касается левого, то оно вытекает из соотношений (14) и (10). Действительно, если $f_{a}$ определяется равенством (13), то

$$
\begin{aligned}
\kappa_{a}(t) & \geqslant \kappa_{a}\left(\sqrt{\left[t^{2}\right]}\right)=\mathscr{K}\left(\sqrt{\left[t^{2}\right]}, f_{a} ; L_{1}, L_{2}\right) \\
& \geqslant \frac{1}{4}\left\{\int_{0}^{\left[t^{2}\right]} f_{a}^{*}(s) d s+t\left(\int_{\left[t^{2}\right]}^{\infty} f_{a}^{*}(s)^{2} d s\right)^{1 / 2}\right\} \\
& =\frac{1}{4}\left\{\sum_{i=1}^{\left[t^{2}\right]} a_{i}^{*}+t\left(\sum_{i=\left[t^{2}\right]+1}^{\infty}\left(a_{i}^{*}\right)^{2}\right)^{1 / 2}\right\}
\end{aligned}
$$

Предложение доказано.

Далее нам потребуется еще одна аппроксимация функционала $\kappa_{a}(t)$, полученная в работе [29]. Для произвольных $a=\left(a_{k}\right)_{k=1}^{\infty} \in \ell_{2}$ и $t \in \mathbb{N}$ определим 
норму

$$
\|a\|_{Q(t)}=\sup \left\{\sum_{j=1}^{t}\left(\sum_{n \in A_{j}} a_{n}^{2}\right)^{1 / 2}\right\}
$$

где верхняя грань берется по всем разбиениям $\left\{A_{j}\right\}_{j=1}^{t}$ натурального ряда $\mathbb{N}$.

ПРЕДЛОЖЕНИЕ 4. Если $a=\left(a_{k}\right)_{k=1}^{\infty} \in \ell_{2} u t^{2} \in \mathbb{N}, m o$

$$
\|a\|_{Q\left(t^{2}\right)} \leqslant \kappa_{a}(t) \leqslant \sqrt{2}\|a\|_{Q\left(t^{2}\right)} .
$$

ДокАзАтЕльство. Прежде всего, из определения $\|\cdot\|_{Q(t)}$ следует, что

$$
\|a\|_{Q\left(t^{2}\right)} \leqslant\|a\|_{1} \quad \text { и } \quad\|a\|_{Q\left(t^{2}\right)} \leqslant t\|a\|_{2} .
$$

Действительно, первое неравенство очевидно, а для доказательства второго достаточно заметить, что ввиду неравенства Коши-Буняковского для произвольного разбиения $\left\{A_{j}\right\}_{j=1}^{t^{2}}$ множества натуральных чисел выполнено

$$
\sum_{j=1}^{t^{2}}\left(\sum_{n \in A_{j}} a_{n}^{2}\right)^{1 / 2} \leqslant t\left(\sum_{j=1}^{t^{2}} \sum_{n \in A_{j}} a_{n}^{2}\right)^{1 / 2}=t\|a\|_{2}
$$

Следовательно,

$$
\begin{aligned}
\kappa_{a}(t) & =\inf \left\{\|b\|_{1}+t\|c\|_{2}: b+c=a, b \in \ell_{1}, c \in \ell_{2}\right\} \\
& \geqslant \inf \left\{\|b\|_{Q\left(t^{2}\right)}+\|c\|_{Q\left(t^{2}\right)}: b+c=a, b \in \ell_{1}, c \in \ell_{2}\right\} \geqslant\|a\|_{Q\left(t^{2}\right)},
\end{aligned}
$$

и левое неравенство в (16) доказано.

Докажем противоположное неравенство. С помощью стандартных рассуждений нетрудно показать, что для каждого $t>0$ сопряженным к пространству $\ell_{1}+\ell_{2}$ с нормой $\kappa_{a}(t)$ будет пространство $\ell_{2} \cap \ell_{\infty}$ с нормой $\max \left\{\|a\|_{\infty}, t^{-1}\|a\|_{2}\right\}$. Тем самым по теореме Хана-Банаха

$$
\kappa_{a}(t)=\max \left\{\sum_{k=1}^{\infty} a_{k} b_{k}: b=\left(b_{k}\right)_{k=1}^{\infty} \in \ell_{2}, \max \left\{\|b\|_{\infty}, t^{-1}\|b\|_{2}\right\} \leqslant 1\right\} .
$$

Поэтому существует такая последовательность $b \in \ell_{2}$, что

$$
\kappa_{a}(t)=\sum_{k=1}^{\infty} a_{k} b_{k} \quad \text { и } \quad \max \left\{\|b\|_{\infty}, t^{-1}\|b\|_{2}\right\}=1 .
$$

Выберем $n_{0}, n_{1}, n_{2}, \ldots, n_{t^{2}} \in\{0,1, \ldots, \infty\}$ по индукции следующим образом: если $0=n_{0}<n_{1}<\cdots<n_{m}$ уже найдены, то

$$
n_{m+1}=1+\max \left\{k: \sum_{n=n_{m}+1}^{k} b_{n}^{2} \leqslant 1\right\} \text {. }
$$

Так как $\|b\|_{\infty} \leqslant 1$, то $\sum_{n=n_{m}+1}^{n_{m+1}} b_{n}^{2} \leqslant 2$. Из того, что $\|b\|_{2} \leqslant t$, следует: $n_{t^{2}}=\infty$. Таким образом,

$$
\kappa_{a}(t)=\sum_{k=1}^{\infty} a_{k} b_{k} \leqslant \sum_{m=1}^{t^{2}}\left(\sum_{n=n_{m-1}+1}^{n_{m}} b_{n}^{2}\right)^{1 / 2}\left(\sum_{n=n_{m-1}+1}^{n_{m}} a_{n}^{2}\right)^{1 / 2} \leqslant \sqrt{2}\|a\|_{Q\left(t^{2}\right)},
$$

и утверждение доказано. 
ДокАЗАТЕЛЬСтво тЕОРЕмЫ 7. Правое неравенство в (8) - непосредственное следствие определения $\mathscr{K}$-функционала и неравенств Хинчина. Действительно, для произвольного $\varepsilon>0$ найдем $b \in \ell_{1}$ и $c \in \ell_{2}$ такие, что $\|b\|_{1}+$ $\sqrt{t}\|c\|_{2} \leqslant \kappa_{a}(\sqrt{t})+\varepsilon$. Тогда, применяя (1) (с учетом оценки для константы), получим

$$
\begin{aligned}
\left\|\sum_{k=1}^{\infty} a_{k} r_{k}\right\|_{t} & \leqslant\left\|\sum_{k=1}^{\infty} b_{k} r_{k}\right\|_{t}+\left\|\sum_{k=1}^{\infty} c_{k} r_{k}\right\|_{t} \\
& \leqslant \sum_{k=1}^{\infty}\left|b_{k}\right|+\sqrt{t}\left(\sum_{k=1}^{\infty} c_{k}^{2}\right)^{1 / 2} \leqslant \kappa_{a}(\sqrt{t})+\varepsilon .
\end{aligned}
$$

Левое неравенство докажем сначала в случае, когда $t \in \mathbb{N}$. По определению $Q(t)$ выберем попарно дизъюнктные множества $A_{1}, \ldots, A_{t}$ в множестве натуральных чисел такие, что

$$
\|a\|_{Q(t)} \leqslant \frac{3}{2}\left\{\sum_{j=1}^{t}\left(\sum_{k \in A_{j}} a_{k}^{2}\right)^{1 / 2}\right\}
$$

Обозначим $\xi_{j}=\sum_{k \in A_{j}} a_{k} r_{k}(j=1, \ldots, t)$. Очевидно, что
$\sum_{k=1}^{\infty} a_{k} r_{k}=\sum_{j=1}^{t} \xi_{j}$.

Введем множества $B_{j}=\left\{t: \xi_{j}(t) \geqslant\left\|\xi_{j}\right\|_{2} / 2\right\}$. Так как $\sum_{j=1}^{t} \xi_{j} \geqslant \frac{1}{2} \sum_{j=1}^{t}\left\|\xi_{j}\right\|_{2}$ на множестве $B=\bigcap_{j=1}^{t} B_{j}$, то

$$
\left\|\sum_{j=1}^{t} \xi_{j}\right\|_{t} \geqslant \frac{1}{2} \sum_{j=1}^{t}\left\|\xi_{j}\right\|_{2} \lambda(B)^{1 / t}
$$

Ввиду независимости и симметричности распределения функций $\xi_{j}$, а также неравенства Пэли-Зигмунда в случае функций Радемахера (см., например, [30])

$$
\lambda(B)=\prod_{j=1}^{t} \lambda\left(B_{j}\right)=2^{-t} \prod_{j=1}^{t} \lambda\left\{t: \xi_{j}(t)^{2} \geqslant \frac{\left\|\xi_{j}\right\|_{2}^{2}}{4}\right\} \geqslant 32^{-t} .
$$

Поэтому, учитывая неравенства (18) и (19), а также предложение 4, получаем:

$$
\left\|\sum_{k=1}^{\infty} a_{k} r_{k}\right\|_{t}=\left\|\sum_{j=1}^{t} \xi_{j}\right\|_{t} \geqslant \frac{1}{64} \sum_{j=1}^{t}\left\|\xi_{j}\right\|_{2} \geqslant \frac{1}{96}\|a\|_{Q(t)} \geqslant \frac{1}{96 \sqrt{2}} \kappa_{a}(\sqrt{t}) .
$$

Если $t \geqslant 1$ произвольно, то ввиду вогнутости $\mathscr{K}$-функционала, а также элементарного неравенства $\sqrt{t} \leqslant 2 \sqrt{[t]}$ находим, что

$$
\frac{1}{192 \sqrt{2}} \kappa_{a}(\sqrt{t}) \leqslant \frac{1}{96 \sqrt{2}} \kappa_{a}(\sqrt{[t]}) \leqslant\left\|\sum_{k=1}^{\infty} a_{k} r_{k}\right\|_{[t]} \leqslant\left\|\sum_{k=1}^{\infty} a_{k} r_{k}\right\|_{t}
$$

и теорема 7 доказана. 
ПримеР 1. Применяя неравенства (9) и (8), нетрудно показать, например, что

$$
\left\|\sum_{k=1}^{\infty} \frac{r_{k}}{k}\right\|_{t} \asymp \ln (1+t) \quad \text { и } \quad\left\|\sum_{k=1}^{n} r_{k}\right\|_{t} \asymp \sqrt{n \min (n, t)} \quad(n \in \mathbb{N}) .
$$

Из теоремы 7 и предложения 3 вытекает следующее соотношение, существенно уточняющее неравенства Хинчина (1).

СлеДСтвиЕ 1. Существует такая универсальная константа $\gamma^{\prime}>0$, что для всех $p \geqslant 1$ и $а=\left(a_{k}\right)_{k=1}^{\infty} \in \ell_{2}$ имеют место неравенства

$$
\gamma^{\prime}\left\{\sum_{i \leqslant p} a_{i}^{*}+\sqrt{p}\left(\sum_{i>p}^{\infty}\left(a_{i}^{*}\right)^{2}\right)^{1 / 2}\right\} \leqslant\left\|\sum_{k=1}^{\infty} a_{k} r_{k}\right\|_{p} \leqslant \sum_{i \leqslant p} a_{i}^{*}+\sqrt{p}\left(\sum_{i>p}^{\infty}\left(a_{i}^{*}\right)^{2}\right)^{1 / 2},
$$

где $\left(a_{i}^{*}\right)_{i=1}^{\infty}-$ невозрастающая перестановка последовательности $\left(\left|a_{n}\right|\right)_{n=1}^{\infty}$.

3.3. Теорема Латалы. Оказывается, аналог неравенств Хитченко справедлив и для общих систем независимых функций; только в этом случае $\mathscr{K}$-функционал в паре $\left(\ell_{1}, \ell_{2}\right)$ должен быть заменен на некоторый специальный функционал Орлича. Для точной формулировки этого интересного общего результата, доказанного Р. Латалой [31] в 1997 г., нам понадобятся некоторые определения.

Если $p>0$, то $\phi_{p}(t)=|1+t|^{p}(t \in \mathbb{R})$ и $\Phi_{p}(f)=\mathrm{E} \phi_{p}(f)$ для произвольной с. в. $f$. Кроме того, если $\left\{f_{k}\right\}_{k=1}^{\infty}-$ последовательность неотрицательных (или симметрично распределенных) с. в., то

$$
\|\|\left(f_{k}\right) \|\left.\right|_{p}:=\inf \left\{t>0: \sum_{k=1}^{\infty} \ln \Phi_{p}\left(\frac{f_{k}}{t}\right) \leqslant p\right\} .
$$

Теорема 8. (а) Пусть $\left\{f_{k}\right\}_{k=1}^{\infty}-$ последовательность независимых неотрицательных с.в. Тогда для любого $n \in \mathbb{N}$

$$
\frac{e-1}{2 e^{2}}\left|\left\|\left(f_{k}\right)\right\|\right|_{p} \leqslant\left\|\sum_{k=1}^{n} f_{k}\right\|_{p} \leqslant e\left|\left\|\left(f_{k}\right)\right\|\right|_{p}, \quad \text { если } p \geqslant 1
$$

$u$

$$
\frac{\left(e^{p}-1\right)^{1 / p}}{2 e^{2}}\left|\left\|\left(f_{k}\right)\right\|\right|_{p} \leqslant\left\|\sum_{k=1}^{n} f_{k}\right\|_{p} \leqslant e\left|\left\|\left(f_{k}\right)\right\|\right|_{p}, \quad \text { если } 0<p \leqslant 1 .
$$

(b) Пусть $\left\{f_{k}\right\}_{k=1}^{\infty}$ - последовательность независимых симметрично распределенных с.в. Тогда, если $p \geqslant 2$, то для любого $n \in \mathbb{N}$

$$
\frac{e-1}{2 e^{2}}\left|\left\|\left(f_{k}\right)\right\|\right|_{p} \leqslant\left\|\sum_{k=1}^{n} f_{k}\right\|_{p} \leqslant e\left|\left\|\left(f_{k}\right)\right\|\right|_{p} .
$$

Мы докажем первые два неравенства этой теоремы, относящиеся к случаю неотрицательных с. в. Доказательство неравенства (23) совершенно аналогично, отличаясь лишь чуть большей технической сложностью (см. [31]). Начнем с простых лемм. 
ЛЕмма 3. Если с.в. $f_{1}, \ldots, f_{n}$ неотрицательны и независимы, то

$$
\Phi_{p}\left(f_{1}+\cdots+f_{n}\right) \leqslant \Phi_{p}\left(f_{1}\right) \cdots \Phi_{p}\left(f_{n}\right) .
$$

ДокАЗАтельство. Ясно, что утверждение достаточно доказать для $n=2$. Но в этом случае оно вытекает из неравенства $\phi_{p}(u+v) \leqslant \phi_{p}(u) \phi_{p}(v)(u, v \geqslant 0)$.

ЛЕмма 4. Если с.в. $f$ и $g$ неотрицательны и независимы, то

$$
\Phi_{p}\left(2 f+\Phi_{p}(f)^{2 / p} g\right) \geqslant \Phi_{p}(f) \Phi_{p}(g) .
$$

ДокАзАтельство. Прежде всего, легко показать (возводя в степень $1 / p$ ), что $\phi_{p}(u v) \geqslant u^{p / 2} \phi_{p}(v)$, если $u \geqslant 1, v \geqslant 1$. Поэтому, учитывая неотрицательность $f$, получим:

$$
\mathrm{E} \phi_{p}\left(2 f+\Phi_{p}(f)^{2 / p} g\right) \chi_{\{g \geqslant 1\}} \geqslant \mathrm{E} \phi_{p}\left(\Phi_{p}(f)^{2 / p} g\right) \chi_{\{g \geqslant 1\}} \geqslant \Phi_{p}(f) \mathrm{E} \phi_{p}(g) \chi_{\{g \geqslant 1\}} .
$$

В то же время, так как $\Phi_{p}(f) \geqslant 1$, то в случае $0 \leqslant u<1, v \geqslant 0$

$$
\phi_{p}\left(2 v+\Phi_{p}(f)^{2 / p} u\right) \geqslant \phi_{p}((1+u) v+u)=\phi_{p}(u) \phi_{p}(v) .
$$

Следовательно,

$$
\mathrm{E} \phi_{p}\left(2 f+\Phi_{p}(f)^{2 / p} g\right) \chi_{\{g<1\}} \geqslant \Phi_{p}(f) \mathrm{E} \phi_{p}(g) \chi_{\{g<1\}},
$$

и утверждение леммы вытекает из полученных соотношений.

В заключение докажем утверждение, в определенном смысле обратное к утверждению леммы 3.

Лемма 5. Пусть $f_{1}, \ldots, f_{n}$ - неотрицательные независимые с.в., удовлетворяющие условию: $\Phi_{p}\left(f_{1}\right) \cdots \Phi_{p}\left(f_{n}\right) \leqslant e^{p}$. Тогда

$$
\Phi_{p}\left(2 e^{2}\left(f_{1}+\cdots+f_{n}\right)\right) \geqslant \Phi_{p}\left(f_{1}\right) \cdots \Phi_{p}\left(f_{n}\right) .
$$

ДокАЗАТЕЛЬСтво. Положим $g_{k}:=2\left(\Phi_{p}\left(f_{1}\right) \cdots \Phi_{p}\left(f_{k}\right)\right)^{2 / p}\left(f_{1}+\cdots+f_{k}\right)$. Так как функционал $\Phi_{p}-$ монотонно возрастающий, то ввиду условия леммы достаточно показать, что для всех $k=1,2, \ldots$ выполнено:

$$
\Phi_{p}\left(g_{k}\right) \geqslant \Phi_{p}\left(f_{1}\right) \cdots \Phi_{p}\left(f_{k}\right) .
$$

Доказательство проведем по индукции. Справедливость неравенства при $k=1$ следует из очевидной оценки $\Phi_{p}\left(2 \Phi_{p}\left(f_{1}\right)^{2 / p} f_{1}\right) \geqslant \Phi_{p}\left(f_{1}\right)$. Предположим, что оно выполнено для некоторого $k$. Тогда опять ввиду монотонности $\Phi_{p}$, а также по предыдущей лемме

$$
\begin{aligned}
\Phi_{p}\left(g_{k+1}\right) \geqslant & \Phi_{p}\left\{2\left(\Phi_{p}\left(f_{1}\right) \cdots \Phi_{p}\left(f_{k+1}\right)\right)^{2 / p}\left(f_{1}+\cdots+f_{k}\right)\right. \\
& \left.+2\left(\Phi_{p}\left(f_{1}\right) \cdots \Phi_{p}\left(f_{k}\right)\right)^{2 / p} f_{k+1}\right\} \\
\geqslant & \Phi_{p}\left(2 f_{k+1}+\Phi_{p}\left(f_{k+1}\right)^{2 / p} g_{k}\right) \\
\geqslant & \Phi_{p}\left(f_{k+1}\right) \Phi_{p}\left(g_{k}\right) \geqslant \Phi_{p}\left(f_{1}\right) \cdots \Phi_{p}\left(f_{k+1}\right) .
\end{aligned}
$$

Лемма доказана. 
ДоКАЗАТЕЛЬСТво тЕОРЕмы 8. Предположим, что $\sum_{k=1}^{\infty} \ln \Phi_{p}\left(f_{k} / t\right)=p$ или, эквивалентно, $\Phi_{p}\left(f_{1} / t\right) \cdots \Phi_{p}\left(f_{n} / t\right)=e^{p}$. Тогда, с одной стороны, по лемме 3

$$
\Phi_{p}\left(\frac{f_{1}+\cdots+f_{n}}{t}\right) \leqslant e^{p} .
$$

Так как $\phi_{p}(u) \geqslant u^{p}$, если $u \geqslant 0$, то для произвольной неотрицательной с. в. $h$ выполнено неравенство $\Phi_{p}(h) \geqslant\|h\|_{p}^{p}$. Следовательно, из предыдущего неравенства получаем: $\left\|f_{1}+\cdots+f_{n}\right\|_{p} \leqslant$ et, и оценка сверху в (21) и (22) доказана.

С другой стороны, по лемме 5

$$
\Phi_{p}\left(\frac{2 e^{2}\left(f_{1}+\cdots+f_{n}\right)}{t}\right) \geqslant e^{p} .
$$

Кроме того, если $p \geqslant 1$, то $\Phi_{p}(h) \leqslant\left(1+\|h\|_{p}\right)^{p}$; если же $p \leqslant 1$, то $\phi_{p}(u) \leqslant$ $1+|u|^{p}$, и, значит, $\Phi_{p}(h) \leqslant 1+\|h\|_{p}^{p}$. Из последних соотношений, а также из (24) вытекают оценки снизу как в (21), так и в (22). Тем самым в случае неотрицательных с. в. теорема доказана.

ЗАмЕЧАНИЕ 1. Применяя стандартную рандомизацию относительно независимой последовательности функций Радемахера, нетрудно показать (подробнее см. [31; замечание 2]), что неравенство (23) выполнено также для произвольной последовательности $\left\{f_{k}\right\}_{k=1}^{\infty}$ независимых с. в. с нулевым математическим ожиданием.

ЗАмечание 2. Соотношения (21), (22) и (23) можно рассматривать как определенный итог предыдущих исследований, связанных с оценками моментов сумм независимых с. в. Прежде всего, в случае $f_{k}=a_{k} r_{k}$, где $r_{k}$ - функции Радемахера на $[0,1]$, а $a_{k} \in \mathbb{R}(k=1,2, \ldots)$, соотношение (23) приводит к неравенствам (20) (правда, с худшими константами).

Рассмотрим теперь более общий случай. Пусть $f_{k}$ - симметрично распределенная с.в., "хвост" распределения которой логарифмически вогнут, т.е. $\mathrm{P}\left\{\left|f_{k}\right| \geqslant \tau\right\}=e^{-N_{k}(\tau)} \quad(\tau \geqslant 0)$, где функция $N_{k}:[0, \infty) \rightarrow[0, \infty]$ выпукла. Обозначим $N_{k}^{*}(t)=\sup \left\{t s-N_{k}(s): s>0\right\}$. Тогда из соотношения (23) (детали см. в [31]) нетрудно получить следующее обобщение основного результата работы [32]:

$$
\left\|\sum_{k=1}^{\infty} a_{k} f_{k}\right\|_{p} \asymp \inf \left\{t>0: \sum_{i \leqslant p} N_{i}^{*}\left(\frac{p a_{i}^{*}}{t}\right) \leqslant p\right\}+\sqrt{p}\left(\sum_{i>p}\left(a_{i}^{*}\right)^{2}\right)^{1 / 2},
$$

где $p \geqslant 2$, а последовательность независимых и симметрично распределенных с. в. $\left\{f_{k}\right\}$ нормирована таким образом, что $\inf \left\{\tau: \mathrm{P}\left\{\left|f_{k}\right| \geqslant \tau\right\} \leqslant e^{-1}\right\}=1$.

В заключение рассмотрим в определенном смысле противоположную ситуацию. Пусть $f_{k}$ - симметрично распределенная с. в., "хвост" распределения которой является логарифмически выпуклым, т. е. $\mathrm{P}\left\{\left|f_{k}\right| \geqslant \tau\right\}=e^{-M_{k}(\tau)}(\tau \geqslant 0)$, где функция $M_{k}:[0, \infty) \rightarrow[0, \infty)$ вогнута. Тогда, применяя (23) (см. [31]), получаем следующее соотношение, ранее доказанное в работе [33]:

$$
\left\|\sum_{k=1}^{\infty} f_{k}\right\|_{p} \asymp\left(\sum_{k=1}^{\infty} \mathrm{E}\left|f_{k}\right|^{p}\right)^{1 / p}+\sqrt{p}\left(\sum_{k=1}^{\infty} \mathrm{E} f_{k}^{2}\right)^{1 / 2} \quad(p \geqslant 2) .
$$




\section{4. Оператор Круглова в симметричных пространствах}

4.1. Неравенства Розенталя и дизъюнктные суммы. Вернемся к неравенствам Розенталя (6) и (7). Выражения, с которыми в них сравнивается $\left\|\sum_{k=1}^{n} f_{k}\right\|_{p}$, могут быть представлены несколько иначе. Для каждой последовательности $\left\{f_{k}\right\}_{k=1}^{\infty}$ функций, заданных на $[0,1]$, через $\left\{\mathbf{f}_{k}\right\}_{k=1}^{\infty}$ обозначим произвольную последовательность дизъюнктных функций, заданных уже на полуоси $[0, \infty)$ и таких, что для каждого $k=1,2, \ldots$ функции $\mathbf{f}_{k}$ и $f_{k}$ одинаково распределены. Например, можно положить $\mathbf{f}_{k}(t)=f_{k}(t-k+1) \chi_{[k-1, k)}(t)(t>0)$. Тогда функция $\mathbf{f}=\sum_{k=1}^{\infty} \mathbf{f}_{k}$, которую будем называть дизбюнктной суммой функций $f_{k}(k=1,2, \ldots)$, очевидно, обладает следующим свойством:

$$
\lambda\{t>0: \mathbf{f}(t)>\tau\}=\sum_{k=1}^{n} \lambda\left\{t \in[0,1]: f_{k}(t)>\tau\right\} \quad(\tau>0),
$$

где $\lambda$ - мера Лебега. Таким образом, неравенства (6) и (7) соответственно означают, что норма $\left\|\sum_{k=1}^{n} f_{k}\right\|_{p}$ эквивалентна норме $\|\mathbf{f}\|_{L_{p} \cap L_{1}[0, \infty)}$, если $f_{k}$ независимы и неотрицательны, и норме $\|\mathbf{f}\|_{L_{p} \cap L_{2}[0, \infty)}$, если эти функции независимы и имеют нулевое математическое ожидание. Здесь, как обычно,

$$
\|g\|_{L_{p} \cap L_{q}[0, \infty)}=\max \left\{\|g\|_{L_{p}[0, \infty)},\|g\|_{L_{q}[0, \infty)}\right\} .
$$

Иначе говоря, с константами, зависящими лишь от $p$, последовательность $\left\{f_{k}\right\}_{k=1}^{\infty}$ в $L_{p}[0,1]$ эквивалентна последовательности $\left\{\mathbf{f}_{k}\right\}_{k=1}^{\infty}$ либо в пространстве $L_{p} \cap L_{1}[0, \infty)$, либо в пространстве $L_{p} \cap L_{2}[0, \infty)$ в зависимости от условий, которым удовлетворяют функции $f_{k}$.

Приведенная переформулировка неравенств Розенталя приводит к следующему естественному вопросу: для каких функциональных пространств, кроме $L_{p}$, справедливы аналогичные неравенства? В 80-е годы прошлого века был опубликован ряд работ, посвященных проблеме сравнения сумм независимых и дизъюнктных функций в различных пространствах, а также применению этих соотношений к изучению геометрии этих пространств. Так, в 1988 г. Н. Каротерс и С. Дилворс [34] перенесли неравенства (7) на пространства Лоренца $L_{p, q}(1 \leqslant p<\infty, 0<q \leqslant \infty)$ (их определение см. в разделе 2 ). Заметим, что ввиду равенства (25) именно симметричные пространства являются наиболее вероятными кандидатами на выполнение в них обобщенных неравенств Розенталя.

Вскоре У. Джонсон и Г. Шехтман [35], используя неравенство ХоффманаЙоргенсона [36], распространили оба неравенства (6) и (7) на случай общих симметричных пространств (заметим в скобках, что еще раньше интересное соотношение типа (6) было доказано для пространств $L_{p, \infty}$ М. Маркусом и Ж. Пизье [37]). Для того чтобы сформулировать их теорему, введем следующую полезную конструкцию (см. [38] или [17; 2.f]). Если $X$ - симметричное 
пространство на $[0,1]$ и $1 \leqslant p \leqslant \infty$, то множество $Z_{X}^{p}$ состоит из всех измеримых на $(0, \infty)$ функций $f$, для которых

$$
\|f\|_{Z_{X}^{p}}:=\left\|f^{*} \chi_{[0,1]}\right\|_{X}+\left\|f^{*} \chi_{[1, \infty)}\right\|_{L_{p}[1, \infty)}<\infty .
$$

Функционал $\|\cdot\|_{Z_{X}^{p}}$ является лишь квазинормой (неравенство треугольника выполняется с константой). Тем не менее, для любого симметричного пространства $X$ он эквивалентен подходящей симметричной норме. Действительно, так как $X \subset L_{1}[0,1]$, то ввиду известного соотношения (см., например, [28] или [21; упражнение 5.7.3], а также, в случае $p=2$, неравенства $(10)$ при $t=1$ ) $\|f\|_{\left(L_{1}+L_{p}\right)(0, \infty)} \asymp \int_{0}^{1} f^{*}(x) d x+\left(\int_{1}^{\infty}\left(f^{*}(x)\right)^{p} d x\right)^{1 / p}, \quad f \in\left(L_{1}+L_{p}\right)(0, \infty)$,

функционал $\|f\|_{Z_{X}^{p}}$ эквивалентен норме $\|f\|_{Z_{X}^{p}}^{\prime}:=\left\|f^{*} \chi_{[0,1]}\right\|_{X}+\|f\|_{\left(L_{1}+L_{p}\right)(0, \infty)}$. Тем самым $Z_{X}^{p}$ - симметричное пространство на полуоси $[0, \infty)$.

Теорема 9 (Джонсон-Шехтман, [35]). Пусть X - произвольное симметричное пространство на $[0,1]$. Тогда существует такое $C>0$, что для любой последовательности независимых функций $\left\{f_{k}\right\}_{k=1}^{\infty} \subset X, \int_{0}^{1} f_{k}(t) d t=0$ $(k=1,2, \ldots)$, и любой последовательности неотрицательных независимых функций $\left\{g_{k}\right\}_{k=1}^{\infty} \subset X$ выполнено:

$$
\|\mathbf{f}\|_{Z_{X}^{2}} \leqslant C\left\|\sum_{k=1}^{\infty} f_{k}\right\|_{X} \quad u \quad\|\mathbf{g}\|_{Z_{X}^{1}} \leqslant C\left\|\sum_{k=1}^{\infty} g_{k}\right\|_{X},
$$

где $\mathbf{f} u \mathbf{g}$ - дизгюнктные суммы, соответствующие последовательностям $\left\{f_{k}\right\}_{k=1}^{\infty} u\left\{g_{k}\right\}_{k=1}^{\infty}$ соответственно.

Если, кроме того, $X \supset L_{p}$ при некотором $p<\infty$, то существует такое $C_{1}=C_{1}(p)>0$, что для последовательностей с такими же свойствами имеют место противоположные неравенства:

$$
\begin{gathered}
\left\|\sum_{k=1}^{\infty} f_{k}\right\|_{X} \leqslant C_{1}\|\mathbf{f}\|_{Z_{X}^{2}}, \\
\left\|\sum_{k=1}^{\infty} g_{k}\right\|_{X} \leqslant C_{1}\|\mathbf{g}\|_{Z_{X}^{1}} .
\end{gathered}
$$

Как мы увидим, условие "удаленности" $X$ от пространства $L_{\infty}$ из второй части последней теоремы $\left(X \supset L_{p}\right.$ при некотором $\left.p<\infty\right)$ не является необходимым для выполнения неравенств (27) и (28). Они справедливы также для некоторых симметричных пространств, расположенных по "другую сторону" от пространств $L_{p}(p<\infty)$. Это удалось выяснить, используя иной подход, основанный на применении так называемого свойства Круглова.

\section{2. Свойство и оператор Круглова: первоначальные сведения.} Свойство Круглова было введено М.Ш. Браверманом [39] при изучении сравнения сумм независимых и дизъюнктных функций в симметричных пространствах. Название объясняется тем, что оно было инспирировано некоторыми 
вероятностными конструкциями В. М. Круглова [40], связанными с изучением безгранично делимых распределений и, в частности, с анализом классической формулы Леви-Хинчина. Пусть $f$ - измеримая функция (случайная величина) на $[0,1]$. Через $\pi(f)$ обозначим с. в. $\sum_{i=1}^{N} f_{i}$, где $f_{i}$ - независимые копии $f$, а $N-$ с. в., независимая относительно последовательности $\left\{f_{i}\right\}$, имеющая распределение Пуассона с параметром 1 . Иначе говоря, $\pi(f)$ - смесь дискретного распределения вероятностей $p_{m}=1 /(e m !), m=0,1,2, \ldots$, и семейства $m$-кратных сверток $\mathscr{F}_{f}^{* m}(x)$ функции распределения $\mathscr{F}_{f}(x)$ данной с. в. $f$. В теории вероятностей такое распределение обычно называют сложным распределением Пуассона [41; гл. 12]. Непосредственные вычисления показывают, что функция распределения и характеристическая функция с. в. $\pi(f)$ определяются равенствами:

$$
\mathscr{F}_{\pi(f)}(x)=\frac{1}{e}\left(\chi_{(0, \infty)}(x)+\mathscr{F}_{f}(x)+\sum_{l=2}^{\infty} \frac{1}{l !} \mathscr{F}_{f}^{* l}(x)\right) \quad(x \in \mathbb{R})
$$

и

$$
\theta_{\pi(f)}(t)=\exp \left(\int_{-\infty}^{\infty}\left(e^{i t x}-1\right) d \mathscr{F}_{f}(x)\right)=\exp \left(\theta_{f}(t)-1\right) \quad(t \in \mathbb{R}),
$$

где $\mathscr{F}_{f}$ и $\theta_{f}$ - соответственно функция распределения и характеристическая функция с. в. $f$.

В [40] было доказано следующее утверждение.

ТеОрема 10 (Круглов). Пусть функиия $\Phi(x)$ непрерьвна на $\mathbb{R}, \Phi(x) \geqslant 0$ $\Phi(0)=0$, а также удовлетворяет одному из следующих условий:
(a) $\Phi(x+y) \leqslant B \Phi(x) \Phi(y) \quad$ или
(b) $\Phi(x+y) \leqslant B(\Phi(x)+\Phi(y))$

для некоторого $B>0$ и всех $x, y \in \mathbb{R}$. Тогда для произвольной с. в. $f$ соотношения $\mathrm{E} \Phi(f)<\infty$ и $\mathrm{E} \Phi(\pi(f))<\infty$ эквивалентны.

ДоказАтельство. Предположим, например, что выполнено условие (а). Пусть Е $\Phi(f)=\int_{0}^{1} \Phi(f(x)) d x:=A<\infty$, a $\left\{f_{k}\right\}_{k=1}^{\infty}$ - последовательность независимых функций, одинаково распределенных с $f$. Тогда по условию для каждого $n \in \mathbb{N}$

$$
\mathrm{E} \Phi\left(\sum_{k=1}^{n} f_{k}\right) \leqslant B \mathrm{E}\left(\Phi\left(\sum_{k=1}^{n-1} f_{k}\right) \Phi\left(f_{n}\right)\right)=B A \mathrm{E} \Phi\left(\sum_{k=1}^{n-1} f_{k}\right) \leqslant \cdots \leqslant B^{n-1} A^{n} .
$$

По определению $\pi(f)$ принимает значения, равные $\sum_{k=1}^{n} f_{k}$ на дизъюнктных множествах $E_{n}, \lambda\left(E_{n}\right)=1 /(e n !)(n=1,2, \ldots)$, причем наборы $\left\{f_{1}, \ldots, f_{n}, \chi_{E_{n}}\right\}$ состоят из независимых функций. Поэтому

$$
\begin{aligned}
\mathrm{E} \Phi(\pi(f)) & =\sum_{n=1}^{\infty} \mathrm{E} \Phi\left(\sum_{k=1}^{n} f_{k} \chi_{E_{n}}\right)=\sum_{n=1}^{\infty} \mathrm{E} \Phi\left(\sum_{k=1}^{n} f_{k}\right) \lambda\left(E_{n}\right) \\
& \leqslant \sum_{n=1}^{\infty} B^{n-1} A^{n} \lambda\left(E_{n}\right)=\frac{1}{e} \sum_{n=1}^{\infty} \frac{B^{n-1} A^{n}}{n !}=\frac{e^{B A}-1}{e B}<\infty .
\end{aligned}
$$


Наоборот, из первого равенства в предыдущей цепочке соотношений следует, что

$$
\mathrm{E} \Phi(\pi(f)) \geqslant \mathrm{E} \Phi\left(f_{1} \chi_{E_{1}}\right)=e^{-1} \mathrm{E} \Phi\left(f_{1}\right),
$$

откуда ввиду одинаковой распределенности функций $f_{1}$ и $f$ получаем неравенство $\mathrm{E} \Phi(f) \leqslant e \mathrm{E} \Phi(\pi(f))$. Теорема доказана.

В частности, если дополнительно $\Phi$ - четная выпуклая функция, то мы можем определить пространство Орлича $L_{\Phi}$ на $[0,1]$ (см. раздел 2$)$ и тогда из теоремы 10 следует, что с. в. $f$ и $\pi(f)$ принадлежат или не принадлежат пространству $L_{\Phi}$ одновременно. В связи с этим возникает естественный вопрос: для каких симметричных пространств верно аналогичное утверждение?

ОПРЕДЕЛЕНИЕ 3. Говорят, что симметричное пространство $X$ на $[0,1]$ имеет свойство Круглова $(X \in \mathbb{K})$, если из того, что $f \in X$, следует: $\pi(f) \in X$.

Заметим, что противоположная импликация: $\pi(f) \in X \Longrightarrow f \in X$ выполнена всегда. Действительно, так как ввиду определения $\lambda\{|\pi(f)| \geqslant \tau\} \geqslant$ $e^{-1} \lambda\{|f| \geqslant \tau\}(\tau>0)$, то, применяя предложение 1 , получаем, что $\|f\|_{X} \leqslant$ $e\|\pi(f)\|_{X}$.

Упрощая ситуацию, можно сказать, что свойством Круглова обладают пространства, достаточно "удаленные" от пространства $L_{\infty}$ (детальное рассмотрение этого вопроса см. в п. 4.3). Прежде всего, по теореме 10, примененной в случае $\Phi(x)=x^{p}$, это пространства $L_{p}$ для всех $1 \leqslant p<\infty$. Более того, аналогичное свойство имеют максимальные симметричные пространства (см. раздел 2), содержащие $L_{p}$ для некоторого $p<\infty$ [39; теорема 1.2] (и в частности, если нижний индекс Бойда пространства $X$ нетривиален, т. е. $\left.\alpha_{X}>0\right)$. В то же время последнее условие не является необходимым; так, экспоненциальное пространство Орлича $\exp L_{p}(p>0)$ имеет свойство Круглова, если и только если $0<p \leqslant 1$. Последнее утверждение является следствием теоремы 10, а также рассуждений из начала п. 2.4 монографии [39] (более точные результаты см. далее в п. 4.3.1).

Свойство Круглова оказалось чрезвычайно полезным средством при изучении целого ряда геометрических свойств банаховых пространств измеримых функций, играя, в частности, центральную роль в монографии [39]. Причина в том, что при некотором дополнительном условии на последовательность независимых функций это свойство гарантирует выполнение неравенств вида (27) и (28).

ТеОрема 11 [39; лемма 1.4]. Пусть симметричное пространство X имеет свойство Круглова. Тогда для произвольной последовательности независимьх функций $\left\{f_{k}\right\}_{k=1}^{\infty} \subset X$ такой, что

выполнено:

$$
\sum_{k=1}^{\infty} \lambda\left\{f_{k} \neq 0\right\} \leqslant 1,
$$

$$
\left\|\sum_{k=1}^{\infty} f_{k}\right\|_{X} \leqslant C\|\mathbf{f}\|_{X},
$$

где $C>0$ зависит только от $X$. 
ЗАмечание 3 . Так как ввиду $(31)\{\mathbf{f} \neq 0\} \subset[0,1]$, то по сравнению с неравенствами (27) и (28) правая часть обобщенного неравенства Розенталя в этом случае значительно упрощается и при его доказательстве достаточно ограничиться рассмотрением неотрицательных функций.

Важно отметить, что для максимальных симметричных пространств верно и обратное утверждение [39; лемма 1.6]: если для произвольной последовательности независимых функций $\left\{f_{k}\right\}_{k=1}^{\infty} \subset X$, удовлетворяющей условию (31), выполнено (32), то $X \in \mathbb{K}$. Эти результаты подсказывают, как могут быть получены необходимые и достаточные условия, при которых в симметричном пространстве $X$ справедливы неравенства (27) и (28). Главная проблема, возникающая при этом, состоит в распространении теоремы 11 на общие последовательности независимых функций (как неотрицательных, так и имеющих нулевое среднее), т. е. в отказе от условия (31). Она была решена авторами настоящего обзора [42], [43] на основе операторного подхода. На пространстве всех п. в. конечных измеримых по Лебегу на $[0,1]$ функций был определен линейный положительный оператор $\mathscr{K}$, тесно связанный со свойством Круглова (и поэтому названный там также оператором Круглова). Введенное понятие, во-первых, позволило использовать многочисленные преимущества операторного языка, например, применять теорию интерполяции операторов. Во-вторых, оказалось возможным рассмотреть более общую ситуацию, когда нормы в левой и правой частях неравенств (27) и (28) берутся в разных пространствах. И наконец, удалось достаточно естественно и просто решить вопрос о точности (по порядку) констант в неравенствах типа Розенталя [44]. В заключение этого пункта приведем детальное изложение этой конструкции, с помощью которой далее будут получены практически окончательные результаты о сравнении сумм независимых и дизъюнктных функций в симметричных пространствах.

Определим сначала вспомогательный оператор $\mathscr{K}_{1}$ со значениями в пространстве $S(\Omega, \mathscr{P})$, где $(\Omega, \mathscr{P}):=\prod_{k=0}^{\infty}\left([0,1], \lambda_{k}\right) \quad\left(\lambda_{k}-\right.$ мера Лебега на $[0,1]$, $k=0,1, \ldots)$. Пусть $\left\{E_{n}\right\}$ - последовательность попарно дизъюнктных подмножеств отрезка $[0,1], \lambda\left(E_{n}\right)=1 /(e n !)(n \in \mathbb{N})$. Для произвольной $f \in S([0,1], \lambda)$ положим

$$
\mathscr{K}_{1} f\left(\omega_{0}, \omega_{1}, \omega_{2}, \ldots\right):=\sum_{n=1}^{\infty} \sum_{k=1}^{n} f\left(\omega_{k}\right) \chi_{E_{n}}\left(\omega_{0}\right)
$$

Хорошо известно, что существует сохраняющее меру отображение $\delta:(\Omega, \mathscr{P}) \rightarrow$ $([0,1], \lambda)$. Для каждой $g \in S(\Omega, \mathscr{P})$ определим $R(g)(x):=g\left(\delta^{-1} x\right), x \in[0,1]$. Легко видеть, что

$$
\lambda\{x \in[0,1]: R(g)(x)<t\}=\mathscr{P}\{\omega \in \Omega: g(\omega)<t\} \quad(t \in \mathbb{R}) .
$$

Поэтому $\mathscr{K}:=R \mathscr{K}_{1}-$ положительный линейный оператор, действующий в пространстве $S([0,1], \lambda)$, причем для любой измеримой на $[0,1]$ функции $f$ распределения функций $\mathscr{K} f$ и $\mathscr{K}_{1} f$ одинаковы. 
Так как мы рассматриваем симметричные пространства, то для нас важно лишь распределение функции $\mathscr{K} f$, в связи с чем на определение оператора $\mathscr{K}$ можно смотреть с несколько иной (и зачастую более удобной точки зрения). Если $f \in S([0,1], \lambda)$ и $\left\{f_{n, k}\right\}_{k=1}^{n}(n \in \mathbb{N})$ - последовательность наборов функций, измеримых на $[0,1]$, такая, что для каждого $n \in \mathbb{N}$

(i) $f_{n, 1}, \ldots, f_{n, n}, \chi_{E_{n}}-$ последовательность независимых с. в.,

(ii) $\mathscr{F}_{f_{n, k}}=\mathscr{F}_{f}, k=1, \ldots, n$, то положим

$$
\mathscr{K}^{\prime} f(x):=\sum_{n=1}^{\infty} \sum_{k=1}^{n} f_{n, k}(x) \chi_{E_{n}}(x), \quad x \in[0,1] .
$$

Непосредственная проверка показывает, что с. в. $\mathscr{K} f$ и $\mathscr{K}^{\prime} f$ одинаково распределены. Поэтому оператор $\mathscr{K}$ можно в определенном смысле отождествлять с $\mathscr{K}^{\prime}$.

Первый простой результат, относящийся к оператору $\mathscr{K}$, является непосредственным следствием теоремы о замкнутом графике.

Лемма 6. Если $X$ и $Y$ - такие симметричные пространства на [0,1], что $\mathscr{K} f \in Y$ для каждой $f \in X$, то для некоторого $C>0$ выполнено: $\|\mathscr{K} f\|_{Y} \leqslant C\|f\|_{X}$.

Так как для произвольной $f \in S(0,1)$ имеет место равенство $\mathscr{F}_{\mathscr{K}^{\prime} f}(x)=$ $\mathscr{F}_{\pi(f)}(x)(x \in \mathbb{R})$, то из $(30)$ получаем, что

$$
\theta_{\mathscr{K} f}(t)=\theta_{\mathscr{K}^{\prime} f}(t)=\theta_{\pi(f)}(t)=\exp \left(\theta_{f}(t)-1\right) \quad(t \in \mathbb{R}) .
$$

Последнее замечание, а также определение свойства Круглова приводят к следующему утверждению, которое в дальнейшем будет постоянно использоваться.

ЛЕмма 7. Если $X$ - произвольное симметричное пространство на $[0,1]$, то $X \in \mathbb{K}$ тогда и только тогда, когда оператор $\mathscr{K}$ ограниченно действуem 8 \&.

\section{3. Ограниченность оператора Круглова в симметричных прост-}

ранствах. Как будет доказано в следующем разделе, ограниченность оператора Круглова в симметричном пространстве гарантирует выполнение в нем обобщенных неравенств Розенталя (отчасти это показывают уже теорема 11 и лемма 7). Прежде всего, мы уточним результаты [39] об ограниченности оператора $\mathscr{K}$ в экспоненциальных пространствах Орлича. Кроме того, будут найдены необходимые и достаточные условия его ограниченности в пространствах Лоренца, из которых вытекает ряд интересных следствий. В частности, мы покажем, что ограниченность оператора Круглова - это весьма деликатное свойство, которое нельзя охарактеризовать в терминах вложений так, как, например, справедливость неравенств Хинчина в симметричном пространстве (см. начало раздела 7 или, подробнее, [66]). Естественный, казалось бы, "рубикон" - пространство Орлича $\exp L_{1}$ - на самом деле, таковым не является: 
оператор Круглова может быть ограничен в симметричном пространстве, содержащемся в $\exp L_{1}$, и не ограничен в симметричном пространстве, содержащем $\exp L_{1}$.

4.3.1. Экспоненциальные пространства Орлича. Действие оператора Круглова в пространствах $\exp L_{p}$ (определение см. в разделе 2) может быть изучено достаточно полно. Результаты, полученные здесь, будут неоднократно применяться в дальнейшем. Напомним, что $\exp L_{\infty}:=L_{\infty}$.

Уже ввиду теоремы 10 включение $\exp L_{p} \in \mathbb{K}$ выполнено при $0<p \leqslant 1$. Следовательно, по лемме 7 в этом случае оператор Круглова ограничен в пространстве $\exp L_{p}$. В то же время, если $p>1$, то пространство $\exp L_{p}$ не имеет свойства Круглова (см. рассуждения из начала §2.4 монографии [39]), и оператор $\mathscr{K}$ не действует в нем. Мы уточним здесь последнее утверждение, определив наименьшее симметричное пространство, содержащее образ $\mathscr{K}\left(\exp L_{p}\right)$ для $p>1$. Для этого нам понадобится еще одно семейство функций Орлича на $[0, \infty)$ :

$$
M_{p}(t):=e^{t \ln ^{1 / p}(e+t)}-1 \quad(p>0) .
$$

Далее неоднократно будет использоваться тот факт, что для каждого $p>0$ пространство $L_{M_{p}}$ (соответственно $L_{N_{p}}$ ) совпадает с пространством Марцинкевича $M_{\psi_{p}}$ (соответственно $\left.M_{\varphi_{p}}\right)$, где

$$
\psi_{p}(t):=\frac{t \ln (e / t)}{\ln ^{1 / p}\left(\ln \left(e^{e} / t\right)\right)} \quad\left(\text { соответственно } \varphi_{p}(t):=t \ln ^{1 / p}(e / t)\right) .
$$

Для его доказательства нам потребуются следующие эквивалентные выражения для норм пространств Марцинкевича $M_{\psi_{p}}$ и $M_{\varphi_{p}}$ [16; теорема 2.5.3]:

$$
\begin{array}{rlrl}
\|x\|_{M_{\psi_{p}}} & \asymp \sup _{t \in(0,1)} \frac{t}{\psi_{p}(t)} x^{*}(t), & & \left(x \in M_{\psi_{p}}\right), \\
\|x\|_{M_{\varphi_{p}}} \asymp \sup _{t \in(0,1)} \frac{t}{\varphi_{p}(t)} x^{*}(t) & \left(x \in M_{\varphi_{p}}\right) .
\end{array}
$$

Лемма 8. Для произвольного $p>0$ выполнены равенства $L_{M_{p}}=M_{\psi_{p}} u$ $\left(\exp L_{p}=\right) L_{N_{p}}=M_{\varphi_{p}}($ с эквивалентностъю норм $)$.

ДокАзАтельство. Докажем лишь первое из равенств, так как доказательство второго аналогично (и несколько проще). Пусть $p>0$. Достаточно показать, что фундаментальные функции $\phi_{L_{M_{p}}}$ и $\phi_{M_{\psi_{p}}}$ рассматриваемых пространств эквивалентны, а также что функция $\bar{f}_{p}(t):=\psi_{p}(t) / t$ принадлежит пространству Орлича $L_{M_{p}}$ (см. (35)). Так как ввиду [20] и [16; гл. 2, §5]

$\phi_{L_{M_{p}}}(t)=\frac{1}{M_{p}^{-1}(1 / t)} \quad$ и $\quad \phi_{M_{\psi_{p}}}(t)=\frac{\ln ^{1 / p}\left(\ln \left(e^{e} / t\right)\right)}{\ln (e / t)} \quad\left(=\frac{1}{\bar{f}_{p}(t)}\right) \quad(0<t \leqslant 1)$,

то для проверки первого из утверждений достаточно показать, что функции $M_{p}^{-1}(1 / t)$ и $\bar{f}_{p}(t)$ эквивалентны в некоторой окрестности нуля. Легко видеть, что для каждого $c>0$

$$
\lim _{t \rightarrow 0} \frac{\ln ^{1 / p}\left(e+c \ln (e / t) / \ln ^{1 / p}\left(\ln \left(e^{e} / t\right)\right)\right)}{\ln ^{1 / p}\left(\ln \left(e^{e} / t\right)\right)}=1 .
$$


Поэтому можно выбрать такое $\delta>0$, что для всех $t \in(0, \delta)$

$$
M_{p}\left(\frac{1}{2} \bar{f}_{p}(t)\right) \leqslant e^{2 \ln (e / t) / 3} \leqslant \frac{1}{t} \leqslant e^{3 \ln (e / t) / 2} \leqslant M_{p}\left(2 \bar{f}_{p}(t)\right) .
$$

Тем самым, функции $M_{p}^{-1}(1 / t)$ и $\bar{f}_{p}(t)$ эквивалентны на $(0, \delta)$. Кроме того, из первого неравенства слева в (36) следует: $\bar{f}_{p} \in L_{M_{p}}$. Лемма доказана.

Следующее утверждение показывает важность пространства $L_{M_{1}}$ в изучении сумм равномерно ограниченных независимых с. в. (см. в связи с этим следствие 3.5.2 в [45]).

ТЕОРема 12. Пространство $L_{M_{1}}$ минимально среди таких симметричных пространств $Y$, что оператор Круглова $\mathscr{K}$ ограничен из $L_{\infty}$ в $Y$.

ДокАЗАТЕЛьство. Пусть $g:=\mathscr{K} \chi_{[0,1]}$. Из определения оператора $\mathscr{K}$ (см. также (33)) следует, что

$$
g^{*}(t)=k \quad\left(t_{k}<t<t_{k-1}\right), \quad \text { где } t_{k}:=\frac{1}{e} \sum_{i=k}^{\infty} \frac{1}{i !}(k \in \mathbb{N}) \text { и } t_{0}=1 .
$$

Так как по лемме 8 пространство $L_{M_{1}}$ совпадает с пространством Марцинкевича $M_{\psi_{1}}$, для нормы которого выполнено соотношение (35), то достаточно доказать лишь то, что функции $g^{*}(t)$ и $\bar{f}_{1}(t)=\ln (e / t) / \ln \ln \left(e^{e} / t\right)$ эквивалентны в окрестности нуля. Так как $\bar{f}_{1}$ убывает на $(0,1)$, а $g^{*} \equiv k$ на каждом интервале $\left(t_{k-1}, t_{k}\right)(k \in \mathbb{N})$, то для этого, в свою очередь, достаточно показать, что

$$
\frac{1}{2} \leqslant \lim _{k \rightarrow \infty} \frac{\bar{f}_{1}\left(t_{k-1}\right)}{k} \leqslant \lim _{k \rightarrow \infty} \frac{\bar{f}_{1}\left(t_{k}\right)}{k} \leqslant 1 .
$$

Прежде всего, для каждого $k \in \mathbb{N}$

$$
\begin{aligned}
k ! t_{k} & =\frac{1}{e}\left(1+\sum_{i=1}^{\infty} \frac{1}{(k+1)(k+2) \cdots(k+i)}\right) \\
& \leqslant \frac{1}{e}\left(1+\frac{1}{k+1}+\sum_{i=1}^{\infty} \frac{1}{(k+i)(k+i+1)}\right) \leqslant \frac{1}{e}\left(1+\frac{2}{k+1}\right) \leqslant \frac{2}{e},
\end{aligned}
$$

откуда $1 /(e k !) \leqslant t_{k} \leqslant 2 /(e k !)$ для всех $k \in \mathbb{N}$. Так как по формуле Стирлинга $k ! \asymp \sqrt{2 \pi k} k^{k} e^{-k}$, то тем самым для достаточно больших $k$ справедливо неравенство $k^{-k} \leqslant t_{k}<t_{k-1} \leqslant(k-1)^{-k / 2}$. В итоге элементарные вычисления показывают, что

$$
\frac{1}{2}=\lim _{k \rightarrow \infty} \frac{\bar{f}_{1}\left((k-1)^{-k / 2}\right)}{k} \leqslant \lim _{k \rightarrow \infty} \frac{\bar{f}_{1}\left(t_{k-1}\right)}{k} \leqslant \lim _{k \rightarrow \infty} \frac{\bar{f}_{1}\left(t_{k}\right)}{k} \leqslant \lim _{k \rightarrow \infty} \frac{\bar{f}_{1}\left(k^{-k}\right)}{k}=1 .
$$

Теорема доказана.

Результат теоремы 12 распространяется на все значения $p \in(1, \infty)$.

Теорема 13. Для произвольного $p \in(1, \infty)$ пространство $L_{M_{q}}$, где $1 / p+$ $1 / q=1$, минимально среди таких симметричных пространств $Y$, что оператор Круглова $\mathscr{K}$ ограничен из $\exp L_{p}$ в $Y$. 
ДоказАтельство. Из теорем 10 и 12, а также леммы 7 следует, что оператор $\mathscr{K}$ ограниченно действует из $L_{\infty}$ в $L_{M_{1}}$ и ограничен в пространстве $\exp L_{1}$ $\left(=L_{N_{1}}\right)$. Применяя вещественный метод интерполяции (см. раздел 2 или [21; гл. 3]), получим, что

$$
\mathscr{K}:\left(L_{\infty}, L_{N_{1}}\right)_{\theta, \infty} \rightarrow\left(L_{M_{1}}, L_{N_{1}}\right)_{\theta, \infty} \quad(0<\theta<1) .
$$

По лемме 8 пространства Орлича $L_{N_{1}}$ и $L_{M_{1}}$ совпадают с пространствами Марцинкевича $M_{\varphi_{1}}$ и $M_{\psi_{1}}$ соответственно. Аналогичное свойство имеет и пространство $L_{\infty}$, а именно, $L_{\infty}=M_{\mathrm{id}}$, где $\mathrm{id}(t)=t(t \in[0,1])$. Поэтому, согласно известному описанию пространств вида $\left(M_{\varphi}, M_{\psi}\right)_{\theta, \infty}$ (см. [46; пример 7.1.3]),

$$
\left(L_{\infty}, L_{N_{1}}\right)_{\theta, \infty}=L_{N_{\theta-1}}, \quad\left(L_{M_{1}}, L_{N_{1}}\right)_{\theta, \infty}=L_{M_{(1-\theta)-1}} .
$$

Полагая $p=\theta^{-1}$, отсюда сразу заключаем, что $\mathscr{K}$ непрерывен из $\exp L_{p}=L_{N_{p}}$ в $L_{M_{q}}$. Таким образом, ввиду соотношений (35) доказательство будет закончено, как только мы покажем, что для некоторой константы $C>0$ (возможно, зависящей от $p)$ и всех достаточно малых $t>0$ выполнено $h_{0}(t) \leqslant C\left(\mathscr{K} g_{0}\right)^{*}(t)$, где $g_{0}(t):=\ln ^{1 / p}(e / t)$ и $h_{0}(t):=\ln (e / t) / \ln ^{1 / q}\left(\ln \left(e^{e} / t\right)\right)$. Так как $h_{0}=h_{0}^{*}$, то последнее неравенство справедливо, если и только если для некоторого $C>0$ и всех достаточно больших $\tau>0$ мы имеем

$$
\lambda\left\{\mathscr{K} g_{0}>\frac{\tau}{C}\right\} \geqslant \lambda\left\{h_{0}>\tau\right\} .
$$

Так как $\lambda\left\{h_{0}>\tau\right\} \leqslant e^{-\tau \ln ^{1 / q} \tau}$, а $\lambda\left\{\mathscr{K} g_{0}>\tau / 3\right\} \geqslant e^{-\tau \ln ^{1 / q} \tau}$, если $\tau$ достаточно велико (подробнее см. доказательство теоремы 4.5 в [42]), то теорема доказана.

В заключение приведем одно полезное необходимое условие ограниченности оператора Круглова. Для каждого $n \in \mathbb{N}$ обозначим $\ln _{n} u:=\ln \ldots \ln u(n$ раз) и $\Phi_{n}(u):=\exp \left(u \ln _{n}\left(c_{n}+u\right)\right)-1$, где константа $c_{n}$ выбрана так, что $\ln _{n} c_{n}=1$. Применяя те же рассуждения, что и в доказательстве леммы 8, нетрудно проверить, что пространство Орлича $L_{\Phi_{n}}$ совпадает с пространством Марцинкевича $M_{\varphi_{n}}$, построенным по функции

$$
\varphi_{n}(u):=\frac{u \ln (e / u)}{\ln _{n+1}\left(e^{c_{n}} / u\right)}, \quad u \in(0,1] .
$$

Действуя примерно так же, как в доказательстве теоремы 13, приходим к следующему результату (подробнее см. [42; теорема 7.2]).

ТеОРема 14. Если оператор $\mathscr{K}$ ограничен в симметричном пространстве $X$, то $L_{\Phi_{n}} \subset X$ для каждого $n \in \mathbb{N}$.

В качестве следствия докажем следующее утверждение, которое далее будет неоднократно использоваться.

Теорема 15. Предположим, что оператор $\mathscr{K}$ ограничен в симметричном пространстве $X$. Тогда $\mathscr{K}$ ограничен в $X_{\circ}$, где $X_{\circ}$ - сепарабельная часть $X$ (см. раздел 2). При этом $\|\mathscr{K}\|_{X_{\circ} \rightarrow X_{\circ}} \leqslant\|\mathscr{K}\|_{X \rightarrow X}$.

$B$ частности, если $X$ сепарабельно и оператор $\mathscr{K}$ ограничен во втором двойственном $X^{\times \times}$, то $\mathscr{K}$ ограничен в $X u\|\mathscr{K}\|_{X \rightarrow X} \leqslant\|\mathscr{K}\|_{X \times \times} \rightarrow X^{\times \times}$. 
ДокАЗАтЕЛьСтво. Поскольку, не ограничивая общности, можно считать, что $X \neq L_{\infty}$, то $X_{\circ}$ сепарабельно. Пусть $x \in X_{\circ}$. Тогда найдется такая последовательность $\left\{x_{n}\right\}_{n=1}^{\infty} \subset L_{\infty}$, что $\left\|x_{n}-x\right\|_{X} \rightarrow 0$. Так как

$$
\left\|\mathscr{K} x_{n}-\mathscr{K} x\right\|_{X}=\left\|\mathscr{K}\left(x_{n}-x\right)\right\|_{X} \leqslant\|\mathscr{K}\|_{X \rightarrow X}\left\|x_{n}-x\right\|_{X},
$$

To

$$
\left\|\mathscr{K} x_{n}-\mathscr{K} x\right\|_{X} \rightarrow 0, \quad \text { если } n \rightarrow \infty .
$$

С одной стороны, по теореме 12 выполнено $\mathscr{K} x_{n} \in L_{M_{1}}(n=1,2, \ldots)$. С другой стороны, легко проверить, что $L_{M_{1}} \subset\left(L_{\Phi_{2}}\right)_{\circ}$, а поскольку по теореме 14 $L_{\Phi_{2}} \subset X$, то $X_{\circ} \supset\left(L_{\Phi_{2}}\right)_{\circ} \supset L_{M_{1}}$. Таким образом, $\mathscr{K} x_{n} \in X_{\circ}(n=1,2, \ldots)$, откуда ввиду (37) получаем, что $\mathscr{K} x \in X_{\circ}$. Так как $X_{\circ}-$ подпространство $X$, то $\|\mathscr{K}\|_{X_{\circ} \rightarrow X_{\circ}} \leqslant\|\mathscr{K}\|_{X \rightarrow X}$.

Второе утверждение теоремы - непосредственное следствие первого, так как по условию $X=X_{\circ}=\left(X^{\times \times}\right)_{\circ}$.

4.3.2. Пространства Лоренца. Следующая теорема, доказанная в работе [42], полностью характеризует пространства Лоренца (определение см. в разделе 2), в которых ограничен оператор Круглова. Напомним, что $\Psi$ - множество всех квазивогнутых на $[0,1]$ функций $\psi(t)$, удовлетворяющих условиям: $\psi(0)=\psi(+0)=0$ и $\psi(1)=1$.

TеОрема 16. Пусть $\psi \in \Psi$. Оператор $\mathscr{K}$ ограниченно действует в пространстве Лоренца $\Lambda_{\psi}$ тогда и только тогда, когда для некоторого $C>0$ выполнено:

$$
\sum_{k=1}^{\infty} \psi\left(\frac{u^{k}}{k !}\right) \leqslant C \psi(u) \quad(0<u \leqslant 1)
$$

ДоказАтельство. Обозначив $f:=\chi_{(0, u]}(u \in(0,1])$, предположим, что последовательности $\left\{f_{n, k}\right\}_{k=1}^{n}(n \in \mathbb{N})$ и $\left\{\chi_{E_{k}}\right\}_{k=1}^{\infty}$ удовлетворяют условиям (i) и (ii) из определения оператора $\mathscr{K}^{\prime}\left(\right.$ см. (33)). Тогда функция $f_{n}:=\sum_{k=1}^{n} f_{n, k}$
имеет биномиальное распределение с параметром $u$, т. е.

$$
\lambda\left\{f_{n}=k\right\}=C_{n}^{k} u^{k}(1-u)^{n-k}, \quad k=0,1, \ldots, n, \quad n \in \mathbb{N},
$$

где $C_{n}^{k}=n ! /(k !(n-k) !)$. Следовательно, $\mathscr{K} f(s)=\sum_{k=1}^{\infty} k \chi_{A_{k}}(s)$ и

$$
\begin{aligned}
\lambda\left(A_{k}\right) & =\sum_{n=k}^{\infty} C_{n}^{k} u^{k}(1-u)^{n-k} \lambda\left(E_{n}\right)=\frac{1}{e} \sum_{n=k}^{\infty} \frac{n !}{k !(n-k) !} u^{k}(1-u)^{n-k} \frac{1}{n !} \\
& =\frac{1}{e} \frac{u^{k}}{(1-u)^{k} k !} \sum_{n=k}^{\infty} \frac{(1-u)^{n}}{(n-k) !}=\frac{1}{e} \frac{u^{k}(1-u)^{k}}{(1-u)^{k} k !} \sum_{n=0}^{\infty} \frac{(1-u)^{n}}{n !}=e^{-u} \frac{u^{k}}{k !} .
\end{aligned}
$$

Это равенство показывает, что функция $\mathscr{K} f$ имеет распределение Пуассона с параметром $u$, совпадающее с распределением функции

$$
h(s):=\sum_{k=1}^{\infty} \chi_{\left(0, \tau_{k}\right]}(s), \quad s \in[0,1]
$$


где $\tau_{k}:=e^{-u} \sum_{i=k}^{\infty} u^{i} / i !(k \in \mathbb{N})$. Поэтому

$$
\|\mathscr{K} f\|_{\Lambda_{\psi}}=\|h\|_{\Lambda_{\psi}}=\sum_{k=1}^{\infty} \int_{0}^{\tau_{k}} d \bar{\psi}(s) \leqslant 2 \sum_{k=1}^{\infty} \psi\left(\tau_{k}\right),
$$

где $\bar{\psi}$ - наименьшая вогнутая мажоранта функции $\psi$ (см. раздел 2 ). Так как $\mathscr{K}$ - положительный оператор, ограниченно действующий из $\Lambda_{\psi}$ в пространство $S(0,1)$, а крайние точки положительной части единичной сферы пространства $\Lambda_{\psi}$ - это нормированные характеристические функции измеримых подмножеств $[0,1]$, то ограниченность $\mathscr{K}$ в $\Lambda_{\psi}$ эквивалентна ограниченности этого оператора на множестве всех таких функций (см. следствие 1 леммы 4.5.2 в [16]). Таким образом, из (39) следует, что $\mathscr{K}$ ограничен в $\Lambda_{\psi}$, если и только если для некоторого $C>0$ выполнено:

$$
\sum_{k=1}^{\infty} \psi\left(\tau_{k}\right) \leqslant C \psi(u) \quad(0<u \leqslant 1) .
$$

Нетрудно показать (см. доказательство теоремы 12 ), что $e^{-1} u^{k} / k ! \leqslant \tau_{k} \leqslant$ $2 u^{k} / k !(k \in \mathbb{N})$. Тем самым, поскольку функция $\psi$ квазивогнута, предыдущее неравенство эквивалентно соотношению (38). Теорема доказана.

Доказательство следующего утверждения аналогично, и поэтому мы его опускаем (см. замечание 5.2 в [42]).

ТеОрема 17. Пусть $\psi \in \Psi$. Оператор $\mathscr{K}$ ограниченно действует из пространства Лорениа $\Lambda_{\psi}$ в пространство Марцинкевича $M_{t / \psi(t)}($ или, на языке теории интерполячии операторов [16; гл. $2, \S 6], \mathscr{K}$ - оператор слабого типа $(\psi, \psi))$, тогда и только тогда, когда

$$
\sup _{u \in(0,1], k \in \mathbb{N}} \frac{k \psi\left(u^{k} / k !\right)}{\psi(u)}<\infty .
$$

Далее мы приведем ряд следствий из теорем 16 и 17. Прежде всего, как уже говорилось, ввиду теоремы 14 возникает естественная гипотеза о том, что $\exp L_{1}$ (точнее, его сепарабельная часть) - минимальное симметричное пространство, в котором ограничен оператор Круглова. В п. 4.3.3 будет показано, что это не так: мы приведем пример обладающих этим свойством пространств Марцинкевича, более узких, чем ехр $L_{1}$. Здесь же мы покажем, что, тем не менее, все пространства Лоренца со свойством Круглова лежат "по одну сторону" от пространства $\exp L_{1}$ [5; теорема 4].

TeOpema 18. Пусть $\psi \in \Psi$. Если $\Lambda_{\psi} \in \mathbb{K}, m o \Lambda_{\psi} \supset \exp L_{1}$.

Докажем сначала следующую лемму.

Лемма 9. Если функиия $\psi \in \Psi$ удовлетворяет условию (38), то

$$
\sum_{k=1}^{\infty} \psi\left(2^{-k}\right) \leqslant A \psi(1)
$$

где $A>0$ зависит лищь от константы $C$ из (38). 
ДокАЗАтельСтво. Ввиду (38), $\sum_{j=1}^{\infty} \psi\left(2^{-i j} j^{-j}\right) \leqslant C \psi\left(2^{-i}\right)(i \in \mathbb{N})$, или

$$
\sum_{j=1}^{\infty} \psi\left(2^{-j\left(i+\left[\log _{2} j\right]\right)}\right) \leqslant C \psi\left(2^{-i}\right) \quad(i \in \mathbb{N}) .
$$

Прямые оценки показывают, что величина

$$
\alpha_{n}:=\operatorname{card}\left\{(i, j) \in \mathbb{N}^{2}: j\left(i+\left[\log _{2} j\right]\right) \leqslant n\right\}
$$

удовлетворяет соотношению $\lim _{n \rightarrow \infty} n^{-1} \alpha_{n}=\infty$. Поэтому существует такое $m \in \mathbb{N}$, что для всех $n \geqslant m$ выполнено неравенство $\alpha_{n} \geqslant(C+1) n$. Так как $\psi$ возрастает, то отсюда и из (42) для произвольного $l>m$ следует:

$$
(C+1) \sum_{n=m}^{l} \psi\left(2^{-n}\right) \leqslant \sum_{i=1}^{l} \sum_{j=1}^{\infty} \psi\left(2^{-j\left(i+\left[\log _{2} j\right]\right)}\right) \leqslant C \sum_{i=1}^{l} \psi\left(2^{-i}\right),
$$

откуда

$$
\sum_{n=m}^{l} \psi\left(2^{-n}\right) \leqslant C \sum_{i=1}^{m-1} \psi\left(2^{-i}\right) .
$$

Неравенство (41) является теперь непосредственным следствием того, что $l>$ $m$ произвольно, а $m$ не зависит от $\psi$. Лемма доказана.

ДоКАЗАТЕЛЬСТво теОРЕмЫ 18. Так как по условию $\Lambda_{\psi} \in \mathbb{K}$, то ввиду теоремы 16 для функции $\psi$ выполнено (38). Применяя лемму 9, получаем (41). Кроме того, ввиду леммы 8 и соотношения (35)

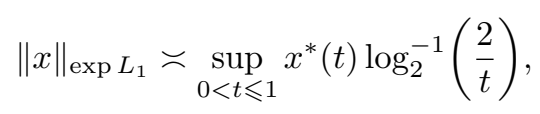

и значит, теорема будет доказана, если показать, что $\log _{2}(2 / t) \in \Lambda_{\psi}$. Действительно,

$$
\begin{aligned}
\left\|\log _{2} \frac{2}{t}\right\|_{\Lambda_{\psi}} & =\int_{0}^{1} \log _{2} \frac{2}{t} d \bar{\psi}(t)=\sum_{k=1}^{\infty} \int_{2^{-k}}^{2^{-k+1}} \log _{2} \frac{2}{t} d \bar{\psi}(t) \\
& \leqslant \sum_{k=1}^{\infty}(k+1)\left(\bar{\psi}\left(2^{-k+1}\right)-\bar{\psi}\left(2^{-k}\right)\right) \leqslant 4+2 \sum_{k=1}^{\infty} \psi\left(2^{-k}\right)<\infty .
\end{aligned}
$$

Теорема доказана.

Теорема 17 позволяет дать прямое (и достаточно короткое) доказательство того, что при выполнении условия $X \supset L_{p}$ для некоторого $p<\infty$ сепарабельное или максимальное симметричное пространство $X$ имеет свойство Круглова. В случае максимальных пространств этот результат был известен ранее (см. [39; теорема 1.2]), однако в его доказательстве в [39] используется теорема 9 Джонсона-Шехтмана. 
Теорема 19. Если симметричное пространство X содержит пространство $L_{p}$ для некоторого $p \in[1, \infty)$, то оператор $\mathscr{K}$ ограниченно действует в пространстве $X^{\times \times}$. В частности, если $X$ сепарабельно или максимально, то $\mathscr{K}$ ограничен в $X$.

Нам здесь также понадобится следующая техническая лемма.

ЛЕмма 10 [47; лемма 11]. Если $\psi$ - возрастающая вогнутая функиия на $[0,1]$ такая, что $\psi(0)=\psi(+0)=0, \psi(1)=1$ u $\psi(t) \leqslant a t^{1 / p}$ для всех $t \in[0,1]$ и некоторьх $p, a \in[1, \infty)$, то

$$
\sup _{0<u \leqslant 1} \frac{1}{\psi(u)} \sum_{k=1}^{\infty} \psi\left(\frac{u^{k}}{k !}\right) \leqslant 5 a p .
$$

ДОКАЗАТЕЛЬСТВо ТЕОРЕМЫ 19. По определению второго ассоциированного пространства

$$
X^{\times \times}=\bigcap \Lambda_{\psi_{y}} \quad \text { и } \quad\|x\|_{X^{\times \times}}=\sup \|x\|_{\Lambda_{\psi_{y}}},
$$

где пересечение и супремум берутся по всем функциям $\psi_{y}$ вида

$$
\psi_{y}(t)=\int_{0}^{t} y^{*}(s) d s \quad(0<t \leqslant 1), \quad y \in X^{\times}, \quad\|y\|_{X^{\times}} \leqslant 1 .
$$

Следуя [47], положим

$$
\theta_{y}(t):=\frac{\psi_{y}(t)+t}{\|y\|_{1}+1} \quad(0<t \leqslant 1), \quad y \in X^{\times}, \quad\|y\|_{X^{\times}} \leqslant 1 .
$$

Очевидно, что $\theta_{y}$ - возрастающая вогнутая функция на $[0,1]$ такая, что $\theta_{y}(0)=$ $\theta_{y}(+0)=0$ и $\theta_{y}(1)=1$ для всех $y \in X^{\times}$. Если $\|y\|_{X \times} \leqslant 1$, то по двойственности

$$
\|y\|_{1}=\int_{0}^{1}|y(t)| d t \leqslant \phi_{X}(1)\|y\|_{X^{\times}} \leqslant 1,
$$

и тогда для каждой $x \in X^{\times \times}$выполнено неравенство $\|x\|_{\Lambda_{\psi}} \leqslant 2\|x\|_{\Lambda_{\theta y}}$. С другой стороны, ввиду (43) и равенства $\phi_{X \times}(x)=1 / \phi_{X}(x)[16 ;$ равенство (2.4.39)]

$$
\begin{aligned}
\|x\|_{\Lambda_{\theta_{y}}} & \leqslant\|x\|_{\Lambda_{\psi_{y}}}+\|x\|_{1} \leqslant\|x\|_{\Lambda_{\psi_{y}}}+\phi_{X^{\times}}(1)\|x\|_{X^{\times \times}} \\
& \leqslant\left(1+\frac{1}{\phi_{X}(1)}\right)\|x\|_{X \times \times}=2\|x\|_{X \times \times} .
\end{aligned}
$$

Из последних соотношений, а также из (43) следует, что

$$
X^{\times \times}=\bigcap \Lambda_{\theta_{y}}, \quad\|x\|_{X \times \times} \asymp \sup \|x\|_{\lambda_{\theta y}},
$$

где пересечение и супремум берутся по всем $\theta_{y}$ вида (44).

Далее, так как $L_{p} \subset X$, то $X^{\times} \subset L_{p}^{\times}=L_{q}$, где $q=p /(p-1)$. Следовательно, для некоторого $a>0$

$\theta_{y}(t) \leqslant \int_{0}^{t} y^{*}(s) d s+t \leqslant\|y\|_{q} t^{1 / p}+t \leqslant a\|y\|_{X \times} t^{1 / p}+t \leqslant(a+1) t^{1 / p} \quad(0<t \leqslant 1)$. 
Тем самым из леммы 10, теоремы 16 и ее доказательства следует, что оператор $\mathscr{K}$ ограничен в пространстве Лоренца $\Lambda_{\theta_{y}}$, если $\|y\|_{X \times} \leqslant 1$, и, кроме того,

$$
\sup _{\|y\|_{X \times 1} \leqslant 1}\|\mathscr{K}\|_{\Lambda_{\theta_{y}} \rightarrow \Lambda_{\theta_{y}}}=C<\infty
$$

Таким образом, ввиду (45) для каждого $x \in X^{\times \times}$имеем:

$$
\|\mathscr{K} x\|_{X^{\times \times}} \asymp \sup _{\|y\|_{X \times} \leqslant 1}\|\mathscr{K} x\|_{\Lambda_{\theta_{y}}} \leqslant C \sup _{\|y\|_{X \times 1} \leqslant 1}\|x\|_{\Lambda_{\theta_{y}}} \asymp\|x\|_{X^{\times \times}}
$$

и первое утверждение теоремы доказано. Если $X$ максимально, то второе утверждение очевидно, а если $X$ сепарабельно, то нужно применить теорему 15. Теорема доказана.

Как уже отмечалось, утверждение, обратное к утверждению последней теоремы, неверно: существуют симметричные пространства со свойством Круглова, не содержащие пространств $L_{p}$ с $p<\infty$. В первую очередь, это экспоненциальные пространства Орлича $\exp L_{p}$ при $0<p \leqslant 1$. Операторный подход и теория интерполяции дают достаточно общий метод для получения подобных примеров.

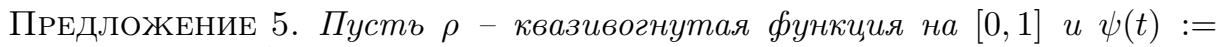
$\rho(t \ln (e / t))$. Если $\tilde{\psi}(t):=t / \psi(t) \quad(0<t \leqslant 1)$, то пространство Марцинкевича $M_{\tilde{\psi}}$ имеет свойство Круглова.

ДокАзАтЕльство. Обозначим через $\ell_{\infty}(1 / \rho)$ пространство всех двусторонних последовательностей $a=\left\{a_{k}\right\}_{k=-\infty}^{\infty}$ с нормой

$$
\|a\|_{l_{\infty}(1 / \rho)}:=\left\|\left\{\frac{a_{k}}{\rho\left(2^{k}\right)}\right\}_{k=-\infty}^{\infty}\right\|_{\ell_{\infty}} .
$$

Так как ввиду теоремы 10 оператор $\mathscr{K}$ ограничен в $L_{1}$ и в $L_{N_{1}}$, то он ограничен в пространстве $\left(L_{1}, L_{N_{1}}\right)_{\ell_{\infty}(1 / \rho)}^{K}$, полученном вещественным методом интерполяции (см. раздел 2). Рассматривая $L_{1}$ и $L_{N_{1}}$ как пространства Марцинкевича и применяя [46; пример 7.1.3] (ср. с доказательством теоремы 13), мы получаем, что $\left(L_{1}, L_{N_{1}}\right)_{\ell_{\infty}(1 / \rho)}^{K}=M_{\tilde{\psi}}$. Предложение доказано.

Приведем еще одно следствие теоремы 17, которое показывает, что ограниченность оператора Круглова в симметричном пространстве не может быть охарактеризована с помощью вложений. Точнее, не существует такого симметричного пространства $X$, что этот оператор ограничен в симметричном пространстве $Y$ тогда и только тогда, когда $Y \subseteq X$.

Теорема 20. Предположим, что функиия $\varphi \in \Psi$ такова, что для каждого $\alpha \in(0,1]$ выполнено: $\sup _{t \in(0,1]} t^{-\alpha} \varphi(t)=\infty$. Тогда существует такая $\psi \in \Psi$, $\psi \leqslant \varphi$, что оператор $\mathscr{K}$ не ограничен ни в одном симметричном пространстве X с фундаментальной функиией $\psi$.

Начнем с леммы, которая будет использоваться и в дальнейшем. 
ЛЕмма 11. Пусть функиия $\varphi \in \Psi$ обладает следующим свойством: для каждой $\psi \in \Psi$ такой, что $\psi \leqslant \varphi$, выполнено условие (40). Тогда существуют $a \geqslant 1 u \alpha \in(0,1]$, для которих $\varphi(t) \leqslant a t^{\alpha}(0 \leqslant t \leqslant 1)$.

ДокАЗАТЕЛьство. Мы используем идею доказательства теоремы 14 из [47]. Предположим, что утверждение леммы неверно, т. е. $\sup _{0<t \leqslant 1} \varphi(t) t^{-1 / n}=\infty$ для каждого $n=1,2, \ldots$. Тогда можно найти такую последовательность $t_{n} \downarrow 0$, что $t_{1}=1$ и

$$
\varphi\left(t_{n}\right) \geqslant \frac{n \varphi\left(t_{n-1}\right)}{t_{n-1}} t_{n}^{1 / n} \quad(n=2,3 \ldots) .
$$

Отсюда

$$
\left(\frac{t_{n-1}}{\varphi\left(t_{n-1}\right)} \varphi\left(t_{n}\right)\right)^{n} \geqslant n^{n} t_{n}>n ! t_{n} .
$$

Если $s_{n}:=t_{n-1} \varphi\left(t_{n}\right) / \varphi\left(t_{n-1}\right)$, то $t_{n}<s_{n}<t_{n-1}$ для всех $n=2,3, \ldots$. Определим функцию

$$
\psi(t)= \begin{cases}\varphi\left(t_{n}\right), & \text { если } t_{n} \leqslant t \leqslant s_{n}, n=1,2,3, \ldots, \\ \frac{t \varphi\left(t_{n-1}\right)}{t_{n-1}}, & \text { если } s_{n} \leqslant t \leqslant t_{n-1}, n=2,3, \ldots, \\ 0, & \text { если } t=0 .\end{cases}
$$

Функция $\psi(t)$ квазивогнута на $[0,1]$ и $\psi \leqslant \varphi$. Ввиду (46) верны равенства $\psi\left(s_{n}^{n} / n !\right)=\psi\left(t_{n}\right)=\psi\left(s_{n}\right)$, и поэтому условие (40) для этой функции не выполнено. Лемма доказана.

ДокАЗАТЕЛЬСтво тЕОРЕмЫ 20. По лемме 11 существует функция $\psi \in \Psi$, $\psi \leqslant \varphi$, которая не удовлетворяет условию (40). Тогда по теореме 17 оператор $\mathscr{K}$ не ограничен из пространства Лоренца $\Lambda_{\psi}$ в пространство Марцинкевича $M_{t / \psi(t)}$. Так как первое из этих пространств - наименьшее из симметричных пространств с фундаментальной функцией $\psi$, а второе - наибольшее (см. раздел 2 или [16; теоремы 2.5.5 и 2.5.7]), то отсюда получаем нужное утверждение.

ЗАмЕчАнИЕ 4. Непосредственное применение теоремы 20 к пространствам Лоренца показывает, что утверждение, обратное к утверждению теоремы 18 , вообще говоря, не верно.

4.3.3. Пространства Марцинкевича, “близкие” к $\exp L_{1}$. Как уже не раз говорилось, пространство Орлича $\exp L_{1}$, построенное по функции $e^{t}-1$, имеет свойство Круглова. По теореме 15 то же самое верно и для его сепарабельной части $\left(\exp L_{1}\right)_{\circ}$. Заметим, что все рассматривавшиеся до сих пор симметричные пространства $X$ со свойством Круглова содержат последнее пространство. Это верно, например, для всех таких пространств Лоренца (см. теорему 18). Ввиду теоремы 14 возникает, казалось бы, естественная гипотеза о том, что $\left(\exp L_{1}\right)$ 。минимальное симметричное пространство, в котором ограничен оператор Круглова. Тем не менее, в работе [5] эта гипотеза была опровергнута. Более того, там доказано, что для всякого симметричного пространства $X \in \mathbb{K}$ существует 
пространство Марцинкевича $M_{\psi} \stackrel{\neq}{\subset} X$, также обладающее свойством Круглова. Основную роль далее будет играть семейство пространств Марцинкевича, определяемое в следующей теореме.

Теорема 21. Существует семейство $\left\{M_{\psi_{\varepsilon}}\right\}_{0<\varepsilon<1}$ пространств Марицнкевича, $M_{\psi_{\varepsilon}} \subseteq M_{\psi_{\delta}}(0<\varepsilon \leqslant \delta<1)$, со следующими свойствами:

1) $M_{\psi_{\varepsilon}} \in \mathbb{K}, 0<\varepsilon<1$;

2) если $X$ симметрично и $X \in \mathbb{K}$, то для всех достаточно мальх $\varepsilon$ мы имеем: $M_{\psi_{\varepsilon}} \subset X$

3) функции $\psi_{\varepsilon}$ попарно не эквивалентны, точнее,

$$
\lim _{t \rightarrow 0} \frac{\psi_{\varepsilon}(t)}{\psi_{\delta}(t)}=0, \quad \text { если } 0<\varepsilon<\delta<1 ;
$$

4) $M_{\psi_{\varepsilon}} \stackrel{\neq}{\subset}\left(\exp L_{1}\right)_{\circ}$ для достаточно мальх $\varepsilon>0$.

Для доказательства нам потребуется следующее простое утверждение.

Лемма 12. Если $f \in L_{1}[0,1]$, mo

$$
\lim _{n \rightarrow \infty} \lambda\left(\operatorname{supp} \mathscr{K}^{n} f\right)=0 .
$$

ДокАзАТЕЛьство. Так как оператор $\mathscr{K}$ положителен, можно считать, что $f \geqslant 0$ и что $\lambda(\operatorname{supp} f)=1$. Тогда, если $a_{n}:=\lambda\left\{t: \mathscr{K}^{n} f(t)=0\right\} \quad(n \in \mathbb{N})$, то по определению оператора $\mathscr{K}$ (см. соотношение (33)) $a_{1}=1 / e$ и

$$
a_{n+1}=\frac{1}{e}+\frac{1}{e} \sum_{k=1}^{\infty} \frac{a_{n}^{k}}{k !}=e^{a_{n}-1} \quad(n=1,2, \ldots) .
$$

Легко видеть, что последовательность $\left\{a_{n}\right\}$ возрастает и $a_{n} \in(0,1)$. Так как $f(x):=e^{x-1}-x$ убывает на $[0,1]$, то функция $e^{x-1}$ имеет на этом отрезке единственную неподвижную точку $x=1$. Следовательно, $\lim _{n \rightarrow \infty} a_{n}=1$, и утверждение доказано.

ДоказАТЕЛЬСтво теОРемЫ 21. Рассмотрим функции $h_{n}:=\left(\mathscr{K}^{n} \chi_{[0,1]}\right)^{*}$, $n \geqslant 0$. Так как оператор $\mathscr{K}$ переводит равноизмеримые функции в равноизмеримые, то

$$
\left(\mathscr{K} h_{n}\right)^{*}=h_{n+1} .
$$

Далее, по лемме 12 имеем $\lambda\left(\operatorname{supp} h_{n}\right) \rightarrow 0$ при $n \rightarrow \infty$, и значит, ряд

$$
g_{\varepsilon}=\sum_{n=0}^{\infty} \varepsilon^{n} h_{n}
$$

сходится всюду на $(0,1]$ для каждого $\varepsilon>0$, а функция $g_{\varepsilon}$ убывает. Кроме того, из определения оператора $\mathscr{K}$ (см. опять (33)) следует, что $\|\mathscr{K}\|_{L_{1} \rightarrow L_{1}}=1$. Поэтому, если $0<\varepsilon<1$, то ряд (49) сходится в $L_{1}$ и $g_{\varepsilon} \in L_{1}$. Покажем теперь, что утверждения теоремы справедливы для семейства $\left\{M_{\psi_{\varepsilon}}\right\}_{\varepsilon>0}$, где $\psi_{\varepsilon}(t)=\int_{0}^{t} g_{\varepsilon}(s) d s \quad(0 \leqslant t \leqslant 1)$. 
1) Докажем, что оператор $\mathscr{K}$ ограничен в $M_{\psi_{\varepsilon}}$. Так как крайние точки единичного шара в этом пространстве равноизмеримы с функцией $g_{\varepsilon}$ [48], [49], то для этого достаточно показать, что $\mathscr{K} g_{\varepsilon} \in M_{\psi_{\varepsilon}}$. Ввиду ограниченности оператора $\mathscr{K}$ в $L_{1}$ имеем:

$$
\mathscr{K} g_{\varepsilon}=\sum_{n=0}^{\infty} \varepsilon^{n} \mathscr{K} h_{n} \prec \sum_{n=0}^{\infty} \varepsilon^{n} h_{n+1} \leqslant \frac{1}{\varepsilon} \sum_{n=0}^{\infty} \varepsilon^{n} h_{n}=\frac{1}{\varepsilon} g_{\varepsilon},
$$

где первое неравенство вытекает из (48) и хорошо известного свойства полуупорядоченности Харди-Литтлвуда (см. раздел 2 и [16; гл. 2, §2]). Отсюда следует, что $\mathscr{K} g_{\varepsilon} \in M_{\psi_{\varepsilon}}$.

2) Предположим теперь, что $X \in \mathbb{K}$. Тогда, в силу леммы 7 и теоремы 15 , $\mathscr{K}: X_{\circ} \rightarrow X_{\circ}$. Поэтому $C=\|\mathscr{K}\|_{X_{\circ} \rightarrow X_{\circ}}<\infty$, откуда следует, что $\left\|h_{n}\right\|_{X} \leqslant$ $C^{n}\left\|\chi_{[0,1]}\right\|_{X}=C^{n}$. Поэтому для всех $\varepsilon<C^{-1}$ ряд (49) сходится в $X_{\circ}$ и $g_{\varepsilon} \in X_{\circ}$.

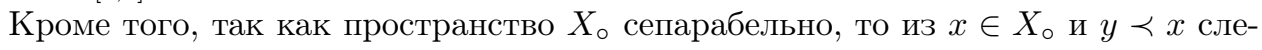
дует: $y \in X_{\text {。 и }}\|y\|_{X_{\circ}} \leqslant\|x\|_{X_{\text {。 }}}$ (см. раздел 2 или [16; теоремы 2.4.3 и 2.4.10]). Таким образом, вместе с $g_{\varepsilon}$ весь единичный шар пространства Марцинкевича $M_{\psi_{\varepsilon}}$ содержится в $X_{\circ}$, откуда $M_{\psi_{\varepsilon}} \subset X_{\circ} \subset X$.

3) Пусть, как и ранее, функция $g_{\varepsilon}$ определяется соотношением (49) и $0<$ $\varepsilon<\delta$. Нетрудно проверить (см. доказательство теоремы 7.2 в [42]), что

$$
\lim _{t \rightarrow 0} \frac{h_{n+1}(t)}{h_{n}(t)}=\infty
$$

Поэтому для любого $m=1,2, \ldots$

$$
\begin{aligned}
\limsup _{t \rightarrow 0} \frac{g_{\varepsilon}(t)}{g_{\delta}(t)} & =\limsup _{t \rightarrow 0}\left(\sum_{n=1}^{\infty} \varepsilon^{n} h_{n}(t)\right)\left(\sum_{n=1}^{\infty} \delta^{n} h_{n}(t)\right)^{-1}=\cdots \\
& =\limsup _{t \rightarrow 0}\left(\sum_{n=m}^{\infty} \varepsilon^{n} h_{n}(t)\right)\left(\sum_{n=m}^{\infty} \delta^{n} h_{n}(t)\right)^{-1} \leqslant\left(\frac{\varepsilon}{\delta}\right)^{m} .
\end{aligned}
$$

Тем самым $\lim _{t \rightarrow 0} g_{\varepsilon}(t) / g_{\delta}(t)=0$, откуда следует $(47)$.

4) Как уже говорилось, $\mathscr{K}$ ограниченно действует в пространстве $\left(\exp L_{1}\right)_{\circ}$. Поэтому достаточно применить уже доказанные утверждения 2) и 3 ).

СлеДСТВИЕ 2. Для любого симметричного пространства $X \in \mathbb{K}$ существует такое симметричное пространство $Y \in \mathbb{K}$, что $Y \stackrel{\neq}{\subset} X$.

\section{5. Сравнение сумм независимых и дизъюнктных функций в симметричных пространствах}

Следующая теорема является уточнением теоремы 1 из [44] (см. также [42; теоремы 3.5 и 6.1]).Ее часть (ii) усиливает и дополняет неравенство ДжонсонаШехтмана (28), в то время как часть (i) показывает практически окончательный характер этого усиления. Через $\mathbf{f}$ всюду далее по-прежнему обозначается дизъюнктная сумма, соответствующая последовательности $\left\{f_{k}\right\}_{k=1}^{\infty}$, а через $L_{M_{p}}$ - пространство Орлича, построенное по функции $M_{p}(t):=e^{t \ln ^{1 / p}(e+t)}-1$ $(p>0)$. 
ТеОРема 22. Пусть $X и Y$ - такие симметричные пространства на $[0,1]$, чmo $X \subseteq Y$.

(i) Предположим, что неравенство

$$
\left\|\sum_{i=1}^{n} f_{i}\right\|_{Y} \leqslant C\|\mathbf{f}\|_{Z_{X}^{1}} \quad\left(=C\|\mathbf{f}\|_{X}\right)
$$

справедливо для некоторого $C>0$ и произвольной последовательности независимых функиий $\left\{f_{k}\right\}_{k=1}^{n} \subset X$, удовлетворяющей условию (31). Тогда оператор Круглова $\mathscr{K}$ ограничен из $X$ в $Y^{\times \times} u\|\mathscr{K}\|_{X \rightarrow Y^{\times \times}} \leqslant C$.

(ii) Eсли оператор $\mathscr{K}$ ограниченно действует из $X$ в $Y$, то для каждой последовательности независимых функииц $\left\{f_{k}\right\}_{k=1}^{n} \subset X$ выполнено:

$$
\left\|\sum_{i=1}^{n} f_{i}\right\|_{Y} \leqslant 8\|\mathscr{K}\|_{X \rightarrow Y}\|\mathbf{f}\|_{Z_{X}^{1}}
$$

В доказательстве части (i) используется одно свойство слабой сходимости распределений. Напомним, что одно из эквивалентных условий слабой сходимости с. в. $f_{n}$ к с. в. $f$ состоит в поточечной сходимости соответствующих характеристических функций: $\theta_{f_{n}}(t) \rightarrow \theta_{f}(t)$ для всех $t \in \mathbb{R}$ (см., например, [50]).

Лемма 13 [39; предложение 5]. Пусть X - максимальное симметричное пространство на $[0,1] u\left\{f_{n}\right\}_{n=1}^{\infty} \subset X$. Если $f_{n}$ слабо сходятся $\kappa f u$

$$
\sup _{n=1,2, \ldots}\left\|f_{n}\right\|_{X}=C<\infty
$$

$m o f \in X u\|f\|_{X} \leqslant C$.

В доказательстве части (ii) нам понадобится аналог известного неравенства Прохорова [51] для неотрицательных функций. Пусть последовательность $\left\{f_{k}\right\}_{k=1}^{\infty}$ состоит из независимых функций, определенных на $[0,1]$. Обозначим через $\left\{h_{k}\right\}_{k=1}^{\infty}$ такую последовательность независимых функций, что $\mathscr{F}_{h_{k}}(x)=\mathscr{F}_{\mathscr{K} f_{k}}(x)(x \in \mathbb{R})$ при всех $k=1,2, \ldots$.

ЛЕмма 14. Если $f_{k}$ независимы и неотрицательны, то для каждого $n \in \mathbb{N}$

$$
\lambda\left\{\sum_{k=1}^{n} f_{k} \geqslant x\right\} \leqslant 2 \lambda\left\{\sum_{k=1}^{n} h_{k} \geqslant \frac{x}{2}\right\} \quad(x>0) .
$$

ДокАЗАТЕЛЬСтво. Без ограничения общности можно предполагать, что $f_{i}$ определены на пространстве $\prod_{i=1}^{n}\left([0,1], \lambda_{i}\right)$, причем $f_{i}\left(t_{1}, \ldots, t_{n}\right)=f_{i}\left(t_{i}\right) \quad(i=$ $1, \ldots, n)$. Так как $f_{i} \geqslant 0$, то из определения оператора Круглова следует, что $\mathscr{K} f_{i} \geqslant g_{i}$ для некоторой с. в. $g_{i}$, одинаково распределенной с функцией $\sigma_{1 / 2} f_{i}$. Следовательно,

$$
\sum_{i=1}^{n} \mathscr{K} f_{i}\left(t_{i}\right) \geqslant \sum_{i=1}^{n} g_{i}\left(t_{i}\right)
$$


С другой стороны, ясно, что для каждого $i=1, \ldots, n$ функция $f_{i}$ одинаково распределена с суммой $g_{i}+g_{i}^{\prime}$, где $g_{i}$ и $g_{i}^{\prime}$ дизъюнктны и одинаково распределены. Поэтому, если $\lambda^{n}=\prod_{i=1}^{n} \lambda_{i}$, то ввиду независимости и соотношения (53) для каждого $x>0$

$$
\begin{aligned}
\lambda^{n}\left\{\sum_{i=1}^{n} f_{i}\left(t_{i}\right)>x\right\} & =\lambda^{n}\left\{\sum_{i=1}^{n}\left(g_{i}\left(t_{i}\right)+g_{i}^{\prime}\left(t_{i}\right)\right)>x\right\} \\
& \leqslant 2 \lambda^{n}\left\{\sum_{i=1}^{n} g_{i}\left(t_{i}\right)>\frac{x}{2}\right\} \leqslant 2 \lambda^{n}\left\{\sum_{i=1}^{n} \mathscr{K} f_{i}\left(t_{i}\right)>\frac{x}{2}\right\} .
\end{aligned}
$$

Поскольку $\mathscr{K} f_{i}$ и $h_{i}$ одинаково распределены, а $h_{i}$ независимы, то (52) доказано.

ДоКАЗАТЕЛЬСТво тЕОРЕмы 22. (i) Мы следуем идее доказательства леммы 1.6 из [39]. Пусть $n \in \mathbb{N}$. Для произвольной $f \in X$ выберем одинаково распределенную с ней функцию $h \in X$ так, что $h$ и $\chi_{[0,1 / n]}$ независимы. Тогда, как легко проверить, функция $h_{n}:=h \chi_{[0,1 / n]}$ будет одинаково распределена с функцией $\sigma_{1 / n} f$. Рассмотрим набор $\left\{\chi_{[0,1 / n]}, h_{n, k}\right\}_{k=1}^{n}$, состоящий из независимых функций, где $\mathscr{F}_{h_{n, k}}(x)=\mathscr{F}_{h_{n}}(x)(x \in \mathbb{R})$ для всех $1 \leqslant k \leqslant n$. Так как функции $\left|\sum_{k=1}^{n} \mathbf{h}_{n, k}\right|$ и $|h|$ имеют одну и ту же функцию распределения, то аналогичное свойство имеют и функции $\left|\sum_{k=1}^{n} \mathbf{h}_{n, k}\right|$ и $|f|$. Кроме того, набор $\left\{h_{n, k}\right\}_{k=1}^{n}$ удовлетворяет неравенству (31). Следовательно, по условию теоремы

$$
\left\|\sum_{k=1}^{n} h_{n, k}\right\|_{Y} \leqslant C\left\|\sum_{k=1}^{n} \mathbf{h}_{n, k}\right\|_{X}=C\|f\|_{X} .
$$

Непосредственные вычисления показывают, что $\theta_{h_{n}}(t)=n^{-1} \theta_{f}(t)+\left(1-n^{-1}\right)$ для всех $t \in \mathbb{R}$. Поэтому характеристическая функция суммы $H_{n}:=\sum_{k=1}^{n} h_{n, k}$
определяется равенством

$$
\theta_{H_{n}}(t)=\left(n^{-1}\left(\theta_{f}(t)-1\right)+1\right)^{n} \quad(t \in \mathbb{R}) .
$$

Так как $\lim _{n \rightarrow \infty} \theta_{H_{n}}(t)=\exp \left(\theta_{f}(t)-1\right)=\theta_{\mathscr{K} f}(t)$ для каждого $t \in \mathbb{R}$, то $H_{n}$ сходится слабо к $\mathscr{K} f$. Тем самым, из соотношения (54), максимальности $Y^{\times \times}$ и леммы 13 следует, что $\|\mathscr{K} f\|_{Y \times \times} \leqslant C\|f\|_{X}$.

(ii) Прежде всего, ясно, что достаточно рассматривать лишь неотрицательные функции. Предположим сначала, что последовательность $\left\{f_{k}\right\}_{k=1}^{\infty} \subset X$ состоит из неотрицательных независимых функций, удовлетворяющих условию (31). Пусть по-прежнему $h_{k}$ независимы и $\mathscr{F}_{h_{k}}(x)=\mathscr{F}_{\mathscr{K} f_{k}}(x)(x \in \mathbb{R})$ при всех $k=1,2, \ldots$. Тогда по лемме 14 имеем (52), и, значит, ввиду предложения 1

$$
\left\|\sum_{k=1}^{n} f_{k}\right\|_{Y} \leqslant 4\left\|\sum_{k=1}^{n} h_{k}\right\|_{Y} .
$$


Так как функции $\mathbf{f}_{k}(k=1, \ldots, n)$ попарно дизъюнктны, то, учитывая определение дизъюнктной суммы, получим

$$
\int_{-\infty}^{\infty}\left(e^{i t x}-1\right) d \mathscr{F}_{\mathbf{f}}(x)=\sum_{k=1}^{n} \int_{-\infty}^{\infty}\left(e^{i t x}-1\right) d \mathscr{F}_{f_{k}}(x)
$$

откуда

$$
\theta_{\mathscr{K} \mathbf{f}}(t)=\prod_{k=1}^{n} \theta_{\mathscr{K} f_{k}}(t)=\prod_{k=1}^{n} \theta_{h_{k}}(t)=\theta_{\sum_{k=1}^{n} h_{k}}(t) \quad(t \in \mathbb{R}) .
$$

Таким образом, функции $\sum_{k=1}^{n} h_{k}$ и $\mathscr{K} \mathbf{f}$ одинаково распределены. Следовательно, ввиду (55)

$$
\left\|\sum_{k=1}^{n} f_{k}\right\|_{Y} \leqslant 4\left\|\sum_{k=1}^{n} h_{k}\right\|_{Y}=4\|\mathscr{K} \mathbf{f}\|_{Y} \leqslant 4\|\mathscr{K}\|_{X \rightarrow Y}\|\mathbf{f}\|_{X}
$$

и в рассматриваемом случае неравенство (51) доказано.

Пусть теперь $\left\{f_{k}\right\}_{k=1}^{\infty} \subset X$ - произвольная последовательность неотрицательных независимых функций. Без ограничения общности мы можем (и будем) предполагать, что $\lambda\left\{f_{i}=\tau\right\}=0$ для каждого $\tau \geqslant 0$ и всех $i=1, \ldots, n$. Тогда существуют такие $0=t_{n}<t_{n-1}<\cdots<t_{1}<t_{0}=\infty$, что

$$
\sum_{i=1}^{n} \lambda\left\{t_{j} \leqslant f_{i}<t_{j-1}\right\}=1 \quad(j=1, \ldots, n) .
$$

Заметим, что для последовательности $\left\{f_{i} \chi_{\left\{f_{i}>t_{1}\right\}}\right\}_{i=1}^{n}$ выполнено условие (31). Поэтому, как уже доказано,

$$
\left\|\sum_{i=1}^{n} f_{i} \chi_{\left\{f_{i}>t_{1}\right\}}\right\|_{Y} \leqslant 4\|\mathscr{K}\|\left\|\sum_{i=1}^{n} \mathbf{f}_{i} \chi_{\left\{\mathbf{f}_{i}>t_{1}\right\}}\right\|_{X}=4\|\mathscr{K}\|\left\|\left(\sum_{i=1}^{n} \mathbf{f}_{i}\right)^{*} \chi_{[0,1]}\right\|_{X} .
$$

Каждая из последовательностей $\left\{f_{i} \chi_{\left\{t_{j}<f_{i}<t_{j-1}\right\}}\right\}_{i=1}^{n}(j=2, \ldots, n)$ также удовлетворяет условию (31). Тем самым, ввиду того, что $\left\|\chi_{[0,1]}\right\|_{X}=1$, получим

$$
\begin{aligned}
& \left\|\sum_{i=1}^{n} f_{i} \chi_{\left\{f_{i}<t_{1}\right\}}\right\|_{Y} \leqslant \sum_{j=2}^{n}\left\|\sum_{i=1}^{n} f_{i} \chi_{\left\{t_{j}<f_{i}<t_{j-1}\right\}}\right\|_{Y} \leqslant 4\|\mathscr{K}\| \sum_{j=2}^{n}\left\|\sum_{i=1}^{n} \mathbf{f}_{i} \chi_{\left\{t_{j}<\mathbf{f}_{i}<t_{j-1}\right\}}\right\|_{X} \\
& \leqslant 4\|\mathscr{K}\| \sum_{j=2}^{n} t_{j-1} \leqslant 4\|\mathscr{K}\|\left(\left\|\sum_{i=1}^{n} \mathbf{f}_{i} \chi_{\left\{\mathbf{f}_{i}>t_{1}\right\}}\right\|_{X}+\sum_{j=2}^{n-1}\left\|\sum_{i=1}^{n} \mathbf{f}_{i} \chi_{\left\{t_{j}<\mathbf{f}_{i}<t_{j-1}\right\}}\right\|_{1}\right) \\
& \quad=4\|\mathscr{K}\|\left(\left\|\left(\sum_{i=1}^{n} \mathbf{f}_{i}\right)^{*} \chi_{[0,1]}\right\|_{X}+\sum_{j=2}^{n-1}\left\|\left(\sum_{i=1}^{n} \mathbf{f}_{i}\right)^{*} \chi_{[j-1, j]}\right\|_{1}\right)=4\|\mathscr{K}\|\left\|\sum_{i=1}^{n} \mathbf{f}_{i}\right\|_{Z_{X}^{1}} .
\end{aligned}
$$

Отсюда и из (56) следует (51), и теорема доказана.

Разумеется, если пространство $Y$ максимально, то утверждение части (i) последней теоремы приобретает следующий вид: оператор $\mathscr{K}$ ограничен из $X$ в $Y$ и $\|\mathscr{K}\|_{X \rightarrow Y} \leqslant C$. Это утверждение может быть распространено на случай, когда $X=Y$ сепарабельно. Тем самым мы получаем частичный положительный ответ на вопрос, заданный в $[39 ; \S 1.6$, с. 16$]$. 
Теорема 23. Предположим, что $X$ - такое сепарабельное симметричное пространство на $[0,1]$, что неравенство

$$
\left\|\sum_{i=1}^{n} f_{i}\right\|_{X} \leqslant C\|\mathbf{f}\|_{Z_{X}^{1}} \quad\left(=C\|\mathbf{f}\|_{X}\right)
$$

справедливо для некоторого $C>0$ и произвольной последовательности независимых функиий $\left\{f_{k}\right\}_{k=1}^{\infty} \subset X$, удовлетворяющей условию (31). Тогда оператор $\mathscr{K}$ ограничен в $X$ u $\|\mathscr{K}\|_{X \rightarrow X} \leqslant C$.

ДокАзАтельство. Стандартные рассуждения, основанные на использовании максимальности пространства $X^{\times \times}$, а также перехода к срезкам рассматриваемых функций, приводят к распространению неравенства (57) на последовательности $\left\{f_{k}\right\}_{k=1}^{\infty} \subset X^{\times \times}$. Поэтому по теореме 22 оператор $\mathscr{K}$ ограниченно действует в $X^{\times \times}$и $\|\mathscr{K}\|_{X^{\times \times} \rightarrow X^{\times x}} \leqslant C$. Применяя теорему 15 , получаем утверждение.

ЗАмечание 5. Однако, если $X \neq Y$, то утверждение (i) теоремы 22 не может быть усилено подобным образом даже в случае сепарабельного $Y$. Достаточно рассмотреть следующий случай: $X=L_{\infty}$ и $Y=\left(L_{M_{1}}\right)_{\text {。 }}$.

Из теоремы 22, а также результатов об ограниченности оператора Круглова в симметричных пространствах, полученных в предыдущем разделе, вытекает целый ряд следствий о справедливости неравенства (51) в конкретных пространствах. Приведем здесь лишь одно из них. Пусть $p \in(1, \infty]$ и $q=p /(p-1)$. Так как $\left(L_{M_{q}}\right)_{\circ}$ - наименьшее среди всех симметричных пространств $Y$ со свойством $Y^{\times \times} \supseteq L_{M_{q}}$, а $\exp L_{p} \subset\left(L_{M_{q}}\right)_{\circ}$, то из теорем 22,12 и 13 получаем следующее утверждение.

СлеДСтвиЕ 3. Для произвольного $p \in(1, \infty]$ существует такая константа $C_{p}>0$, что для всех $n \in \mathbb{N}$ и произвольной последовательности независимых функций $\left\{f_{k}\right\}_{k=1}^{n} \subset \exp L_{p}$ выполнено:

$$
\left\|\sum_{k=1}^{n} f_{k}\right\|_{\left(L_{M_{q}}\right) \circ} \leqslant C_{p}\|\mathbf{f}\|_{Z_{\exp L_{p}}^{1}} .
$$

Кроме того, если симметричное пространство $Y$ обладает тем свойством, что для некоторого $C>0$ и произвольной последовательности независимьх функций $\left\{f_{k}\right\}_{k=1}^{n} \subset \exp L_{p}$, удовлетворяющей условию (31), выполнено неравенство

$$
\left\|\sum_{k=1}^{n} f_{k}\right\|_{Y} \leqslant C\|\mathbf{f}\|_{\exp L_{p}}
$$

$m o\left(L_{M_{q}}\right) \subseteq \subseteq$.

Следующий результат, доказанный в [42], является в определенном смысле обращением второго из утверждений теоремы 9 Джонсона-Шехтмана. 
Теорема 24. Предположим, что симметричное пространство X имеет следующее свойство: для каждого максимального симметричного пространства $Y \supset X$ существует такая константа $C>0$, что для всех $n \in \mathbb{N}$ и произвольной последовательности независимых функций $\left\{f_{k}\right\}_{k=1}^{n} \subset Y$, удовлетворяющей условию (31), выполнено неравенство

$$
\left\|\sum_{i=1}^{n} f_{i}\right\|_{Y} \leqslant C\|\mathbf{f}\|_{Y}
$$

где $\mathbf{f}$ - дизбюнктная сумма последовательности $\left\{f_{k}\right\}_{k=1}^{n}$. Тогда $X \supset L_{p} n p u$ некотором $p<\infty$.

ДоказАтельство. Согласно теореме 2.5.7 из [16], $X \subseteq M_{t / \phi_{X}}$, где $\phi_{X}$ - фундаментальная функция пространства $X$. Следовательно, для каждой функции $\psi \in \Psi$ такой, что $\psi \leqslant \phi_{X}$, имеем: $X \subseteq M_{t / \psi(t)}$. Тем самым по условию и теореме 22 , (i) оператор $\mathscr{K}$ ограниченно действует в пространстве $M_{t / \psi(t)}$. Но тогда ввиду теоремы 17 условие (40) выполнено для каждой такой функции $\psi$. Применяя лемму 11 , получаем, что $\phi_{X}(t) \leqslant a t^{\alpha}$ при некоторых $a \geqslant 1$ и $\alpha \in(0,1]$ и для всех $t \in[0,1]$. Последнее неравенство вместе с теоремой 2.5.5 из [16] гарантирует, что

$$
X \supseteq \Lambda_{\phi_{X}} \supseteq \Lambda_{t^{\alpha}}
$$

Так как пространство Лоренца $\Lambda_{t^{\alpha}}$ содержит $L_{p}$, если $p>1 / \alpha$, то теорема доказана.

ЗАмЕчАнИЕ 6. Из доказательства ясно, что последнее утверждение останется верным, если в его формулировке класс всех максимальных симметричных пространств заменить множеством пространств Марцинкевича.

Перейдем теперь к рассмотрению случая независимых функций, в среднем равных нулю. Первоначальный вариант следующей теоремы, усиливающей неравенство Джонсона-Шехтмана (27), был доказан в работе [52] с использованием техники теории безгранично делимых распределений. Здесь доказательство значительно упрощено [53].

Tеорема 25. Пусть $X$ и $Y$-симметричные пространства на $[0,1], X \subset Y$. Тогда, если оператор $\mathscr{K}$ ограниченно действует из $X$ в $Y$, то существует такая универсальная константа $\alpha>0$, что для любой последовательности независимых функиий $\left\{f_{k}\right\}_{k=1}^{\infty} \subset X, \int_{0}^{1} f_{k}(t) d t=0(k=1,2, \ldots)$, и всех $n \in \mathbb{N}$ имеет место неравенство

$$
\left\|\sum_{k=1}^{n} f_{k}\right\|_{Y} \leqslant \alpha\|\mathscr{K}\|_{X \rightarrow Y}\|\mathbf{f}\|_{Z_{X}^{2}} .
$$

Отметим ту ключевую роль, которую играет в доказательстве этой теоремы неравенство Прохорова из теоремы 5. Далее нам понадобятся два вспомогательных утверждения. 
ПРЕДЛОЖЕНИЕ 6. Существует такая универсальная константа $\beta>0$, что для любой последовательности $\left\{f_{k}\right\}_{k=1}^{\infty}$ ограниченных симметрично распределенных независимых функиий выполнено

$$
\left\|\sum_{k=1}^{n} f_{k}\right\|_{L_{M_{1}}} \leqslant \beta\|\mathbf{f}\|_{L_{2} \cap L_{\infty}} \quad(n \in \mathbb{N}) .
$$

ДокАЗАТЕЛЬСтво. Рассмотрим следующее подпространство пространства $L_{2} \cap L_{\infty}(0, \infty):$

$$
\begin{aligned}
\left(L_{2} \cap L_{\infty}\right)_{\mathrm{s}}:=\left\{f \in L_{2} \cap L_{\infty}\right. & : \text { с. в. } f^{(k)}(x):=f(x+k-1)(0 \leqslant x \leqslant 1) \\
& \text { симметрично распределена для каждого } k \geqslant 1\} .
\end{aligned}
$$

Нетрудно проверить, что $\left(L_{2} \cap L_{\infty}\right)_{\mathrm{s}}$ замкнуто в $L_{2} \cap L_{\infty}$ и, следовательно, является банаховым пространством. Определим последовательность линейных операторов

$$
U_{n}:\left(L_{2} \cap L_{\infty}\right)_{\mathrm{s}} \rightarrow L_{M_{1}}(\Omega, \mathscr{P}), \quad U_{n} f\left(t_{1}, \ldots, t_{n}, \ldots\right)=\sum_{k=1}^{n} f\left(k-1+t_{k}\right),
$$

где, как обычно, $(\Omega, \mathscr{P})=\prod_{k=1}^{\infty}\left([0,1], \lambda_{k}\right)$. Очевидно, что $\left\|U_{n}\right\|_{\left(L_{2} \cap L_{\infty}\right)_{\mathrm{s}} \rightarrow L_{M_{1}}} \leqslant n$. Пусть $\mathbf{f}_{k}$ - дизъюнктные копии функций $f\left(k-1+t_{k}\right)(k=1,2, \ldots)$, a $\mathbf{f}^{(n)}=$ $\sum_{k=1}^{n} \mathbf{f}_{k}-$ дизъюнктная сумма первых $n$ функций. Так как функции $f\left(k-1+t_{k}\right)$ симметрично распределены, то по теореме 5

$$
\mathscr{P}\left\{\sum_{k=1}^{n} f\left(k-1+t_{k}\right)>\tau\right\} \leqslant \exp \left(-\frac{\tau}{2\left\|\mathbf{f}^{(n)}\right\|_{\infty}} \operatorname{arcsh} \frac{\tau\left\|\mathbf{f}^{(n)}\right\|_{\infty}}{2\left\|\mathbf{f}^{(n)}\right\|_{2}^{2}}\right) \quad(\tau>0) .
$$

Напомним, что функция $\bar{f}_{1}(t):=\ln (e / t) / \ln \left(\ln \left(e^{e} / t\right)\right)$ "максимальна по перестановке" в пространстве $L_{M_{1}}$ (см. первое из соотношений (35) и лемму 8). Кроме того, как нетрудно проверить, для некоторого $C \geqslant 1$ и всех достаточно больших $\tau>0$ выполнены неравенства

$$
e^{-C^{-1} \tau \ln \tau} \leqslant \lambda\left\{t \in(0,1]: \bar{f}_{1}(t)>\tau\right\} \leqslant e^{-\tau \ln \tau} .
$$

Предположим, что $f \in\left(L_{2} \cap L_{\infty}\right)_{\mathrm{s}},\|f\|_{L_{2} \cap L_{\infty}}=1$ и $n_{0} \in \mathbb{N}$ таково, что $f \chi_{\left(0, n_{0}\right]} \neq 0$. Легко видеть, что правая часть в $(59)$ не превосходит

$$
\exp \left(-\frac{\tau}{2} \operatorname{arcsh} \frac{\tau\left\|f \chi_{\left(0, n_{0}\right]}\right\|_{\infty}}{2}\right)
$$

для всех $n \geqslant n_{0}$ и $\tau>0$. Поэтому, так как $\operatorname{arcsh} t \sim \ln t$ при $t \rightarrow+\infty$, то мы получаем, что $\sup _{n=1,2, \ldots}\left\|U_{n} f\right\|_{L_{M_{1}}}<\infty$ для каждой фиксированной $f \in\left(L_{2} \cap L_{\infty}\right)_{\mathrm{s}}$. Но тогда ввиду принципа Банаха-Штейнгауза нормы $\left\|U_{n}\right\|_{\left(L_{2} \cap L_{\infty}\right)_{\mathrm{s}} \rightarrow L_{M_{1}}}$ $(n \in \mathbb{N})$ равномерно ограничены, и утверждение доказано. 
Лемма 15. Пусть $X$ и $Y$ - симметричные пространства на $[0,1]$ и $\mathscr{K}$ : $X \rightarrow Y$. Тогда $L_{M_{1}} \subset Y$ и, кроме того, существует такая универсальная константа $\gamma>0$, что для всех $x \in L_{M_{1}}$ имеет место неравенство

$$
\|x\|_{Y} \leqslant \gamma\|\mathscr{K}\|_{X \rightarrow Y}\|x\|_{L_{M_{1}}} .
$$

ДокАЗАтЕльство. Если функция $\bar{f}_{1}-$ та же, что и в доказательстве предыдущего утверждения, то, как там отмечалось, для произвольной $x \in L_{M_{1}}$ и для всех $0<t \leqslant 1$ неравенство $x^{*}(t) \leqslant \gamma \bar{f}_{1}(t)\|x\|_{L_{M_{1}}}$ выполнено с некоторой универсальной константой $\gamma>0$. Кроме того, $\bar{f}_{1} \leqslant \mathscr{K}_{[0,1]}$ (см. доказательство теоремы 12), и, значит,

$$
\left\|\bar{f}_{1}\right\|_{Y} \leqslant\left\|\mathscr{K} \chi_{[0,1]}\right\|_{Y} \leqslant\|\mathscr{K}\|_{X \rightarrow Y}\left\|\chi_{[0,1]}\right\|_{X}=\|\mathscr{K}\|_{X \rightarrow Y} .
$$

В итоге получаем

$$
\|x\|_{Y} \leqslant \gamma\left\|\bar{f}_{1}\right\|_{Y}\|x\|_{L_{M_{1}}} \leqslant \gamma\|\mathscr{K}\|_{X \rightarrow Y}\|x\|_{L_{M_{1}}}
$$

Лемма доказана.

ДОКАЗАТЕЛЬСТВо ТЕОРЕмЫ 25. Предположим сначала, что функции $f_{k}$ $(k=1,2, \ldots)$ симметрично распределены. Обозначим $t_{0}:=\mathbf{f}^{*}(1)$, где, как и ранее, $\mathbf{f}$ - соответствующая дизъюнктная сумма. Тогда

$$
\sum_{k=1}^{n} \lambda\left\{s \in[0,1]:\left|f_{k}(s)\right|>t_{0}\right\}=\lambda\left\{s>0:|\mathbf{f}(s)|>t_{0}\right\} \leqslant 1
$$

Если $u_{k}:=f_{k} \chi_{\left\{\left|f_{k}\right|>t_{0}\right\}}, v_{k}:=f_{k} \chi_{\left\{\left|f_{k}\right| \leqslant t_{0}\right\}}(k=1, \ldots, n)$, то $\left\{u_{k}\right\}$ и $\left\{v_{k}\right\}-$ последовательности независимых симметрично распределенных функций и $f_{k}=$

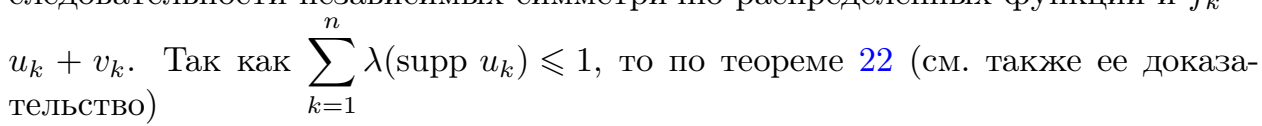

$$
\left\|\sum_{k=1}^{n} u_{k}\right\|_{Y} \leqslant 4\|\mathbf{u}\|_{X}=4\left\|\mathbf{f}^{*} \chi_{[0,1]}\right\|_{X}
$$

Одновременно, применяя лемму 15 и предложение 6 , а также учитывая, что $\left\|\chi_{[0,1]}\right\|_{X}=1$, получаем:

$$
\begin{aligned}
\left\|\sum_{k=1}^{n} v_{k}\right\|_{Y} & \leqslant \gamma\|\mathscr{K}\|_{X \rightarrow Y}\left\|\sum_{k=1}^{n} v_{k}\right\|_{L_{M_{1}}} \leqslant \gamma \beta\|\mathscr{K}\|_{X \rightarrow Y}\|\mathbf{v}\|_{L_{2} \cap L_{\infty}} \\
& \leqslant \gamma \beta\|\mathscr{K}\|_{X \rightarrow Y}\left\|\mathbf{f}^{*} \chi_{[1, \infty)}\right\|_{L_{2} \cap L_{\infty}} \leqslant \gamma \beta\|\mathscr{K}\|_{X \rightarrow Y}\left(t_{0}+\left\|\mathbf{f}^{*} \chi_{[1, \infty)}\right\|_{L_{2}}\right) \\
& \leqslant \gamma \beta\|\mathscr{K}\|_{X \rightarrow Y}\left(\left\|\mathbf{f}^{*} \chi_{[0,1]}\right\|_{X}+\left\|\mathbf{f}^{*} \chi_{[1, \infty)}\right\|_{L_{2}}\right)=\gamma \beta\|\mathscr{K}\|_{X \rightarrow Y}\|\mathbf{f}\|_{Z_{X}^{2}} .
\end{aligned}
$$

Отсюда и из (60) следует, что для симметрично распределенных функций неравенство (58) выполнено с константой $4+\gamma \beta$.

Рассмотрим общий случай, когда известно лишь, что $\int_{0}^{1} f_{k}(t) d t=0 \quad(k=$ $1,2, \ldots)$. Действуя стандартным образом, применим симметризацию. Пусть 
$\left\{f_{k}^{\prime}\right\}_{k=1}^{n}-$ последовательность независимых копий функций $f_{k}(k=1, \ldots, n)$ и, кроме того, $h_{k}:=f_{k}-f_{k}^{\prime}(k=1, \ldots, n)$. Тогда $\left\{h_{k}\right\}_{k=1}^{n}-$ последовательность независимых симметрично распределенных с. в., и, как уже доказано, для нее неравенство (58) выполнено.

Пусть $\mathscr{B}-\sigma$-подалгебра, порожденная последовательностью $\left\{f_{k}\right\}_{k=1}^{n}$, а $\mathrm{E}_{\mathscr{B}}-$ оператор условного математического ожидания, соответствующий ей. Ввиду предложения 2 выполнено равенство $\left\|\mathrm{E}_{\mathscr{B}}\right\|_{Y \rightarrow Y}=1$. Следовательно,

$$
\left\|\sum_{k=1}^{n} f_{k}\right\|_{Y}=\left\|\mathrm{E}_{\mathscr{B}}\left(\sum_{k=1}^{n} h_{k}\right)\right\|_{Y} \leqslant\left\|\sum_{k=1}^{n} h_{k}\right\|_{Y} .
$$

Так как $\lambda\{t>0:|\mathbf{h}(t)|>x\} \leqslant 2 \lambda\{t>0:|\mathbf{f}(t)|>x\} \quad(x \geqslant 0)$, то ввиду предложения 1 неравенство (58) выполнено и в этом случае с константой $\alpha=$ $8+2 \gamma \beta$. Теорема доказана.

\section{6. Неравенства типа Розенталя для симметричных} (квази)норм на последовательностях независимых функций

Пусть $E$ - симметричное (квази)банахово пространство последовательностей (см. раздел 2$),\left\{e_{k}\right\}_{k=1}^{\infty} \subset E$ - стандартный базис в $E$, а $X$ - симметричное пространство функций на $[0,1]$. Для произвольной последовательности неотрицательных независимых функций $\left\{f_{k}\right\}_{k=1}^{n} \subset X(n \in \mathbb{N})$ рассмотрим величину

$$
\|\| \sum_{k=1}^{n} f_{k} e_{k}\left\|_{E}\right\|_{X} .
$$

Основная цель этого раздела - найти как можно более точные оценки для нее в терминах дизъюнктных копий функций данной последовательности. Результаты такого рода находят многочисленные применения при изучении различных вопросов геометрии пространств, например, в локальной теории выпуклых тел [54], [55]. "Происхождение" этой величины очевидно: в случае $E=\ell_{1}$ и $X=L_{p}(1 \leqslant p<\infty)$ она фигурирует в неравенстве Розенталя (6). Мы отдельно рассмотрим оценки снизу и сверху, так как условия, при которых они имеют место, а также методы их доказательства различны.

6.1. Нижние оценки. Мы начнем с одного несложного, но важного утверждения, доказанного в [56; предложение 1] (очень близкий результат см. в [35; лемма 3]).

ПРеДЛОЖеНИЕ 7. Пусть $\left\{f_{k}\right\}_{k=1}^{\infty}-$ последовательность неотрицательных независимых функиий, определенных на $[0,1]$, a $\mathbf{f}$ - соответствующая дизгюнктная сумма. Для любого $t>0$ справедливо неравенство

$$
\frac{1}{2} \lambda\left\{\mathbf{f}^{*} \chi_{[0,1]}>t\right\} \leqslant \lambda\left\{\sup _{k=1,2, \ldots} f_{k}>t\right\} \leqslant \lambda\left\{\mathbf{f}^{*} \chi_{[0,1]}>t\right\} .
$$

ДокАЗАТЕЛЬство. Зафиксируем $t>\mathbf{f}^{*}(1)$ (достаточно рассмотреть лишь этот случай) и обозначим $a_{k}:=\lambda\left\{f_{k}>t\right\} \quad(k=1,2, \ldots)$. Тогда ввиду $(25)$, 
а также независимости функций $f_{k}$

$$
\lambda\left\{\mathbf{f}^{*} \chi_{[0,1]}>t\right\}=\sum_{k=1}^{\infty} a_{k} \leqslant 1 \quad \text { и } \quad \lambda\left\{\sup _{k=1,2, \ldots} f_{k}>t\right\}=1-\prod_{k=1}^{\infty}\left(1-a_{k}\right) .
$$

Нетрудно показать, что

$$
1-\sum_{k=1}^{\infty} a_{k} \leqslant \prod_{k=1}^{\infty}\left(1-a_{k}\right) \leqslant 1-\frac{\sum_{k=1}^{\infty} a_{k}}{1+\sum_{k=1}^{\infty} a_{k}}
$$

Например, правое неравенство является следствием оценок

$$
\prod_{k=1}^{\infty}\left(1-a_{k}\right)\left(1+\sum_{k=1}^{\infty} a_{k}\right) \leqslant \prod_{k=1}^{\infty}\left(1-a_{k}\right)\left(1+a_{k}\right)=\prod_{k=1}^{\infty}\left(1-a_{k}^{2}\right) \leqslant 1 .
$$

Поэтому

$$
\frac{1}{2} \lambda\left\{\mathbf{f}^{*} \chi_{[0,1]}>t\right\} \leqslant \frac{\lambda\left\{\mathbf{f}^{*} \chi_{[0,1]}>t\right\}}{1+\lambda\left\{\mathbf{f}^{*} \chi_{[0,1]}>t\right\}} \leqslant \lambda\left\{\sup _{k=1,2, \ldots} f_{k}>t\right\} \leqslant \lambda\left\{\mathbf{f}^{*} \chi_{[0,1]}>t\right\},
$$

и предложение доказано.

Следующее утверждение было получено в работе [57] (см. также [58], [59]).

Теорема 26. Для произволъных функиионального симметричного пространства $X$ и банахова симметричного пространства последовательностей $E$, для всех $n \in \mathbb{N}$ и любой последовательности неотрицательных независимых функиий $\left\{f_{k}\right\}_{k=1}^{n} \subset X$ справедливо неравенство

$$
\left\|\mathbf{f}^{*} \chi_{[0,1]}\right\|_{X}+\left\|\sum_{k=1}^{n} \mathbf{f}^{*}(k) e_{k}\right\|_{E} \leqslant 6\|\| \sum_{k=1}^{n} f_{k} e_{k}\left\|_{E}\right\|_{X} .
$$

ДокАЗАТЕЛЬство. Сначала мы докажем неравенство (61) в частном случае, когда $X=L_{1}$, а $E$ - пространство последовательностей $\mathbf{s}_{r}(r=1,2, \ldots)$ с нормой $\left\|\left(a_{k}\right)_{k=1}^{\infty}\right\|_{\mathbf{s}_{r}}:=\sum_{k=1}^{r} a_{k}^{\sharp}$, где $\left(a_{k}^{\sharp}\right)_{k=1}^{\infty}-$ невозрастающая перестановка последовательности $\left(\left|a_{k}\right|\right)_{k=1}^{\infty}$.

Обозначим $A(t):=\mathbf{f}^{*}(t)$ и $B(t):=\max _{1 \leqslant k \leqslant n} f_{k}(t)$. Тогда ввиду предложения 7

$$
\frac{1}{2} \int_{0}^{1} A(t) d t \leqslant \int_{0}^{1} B(t) d t \leqslant \int_{0}^{1} A(t) d t .
$$

Далее рассмотрим последовательность $\left\{g_{k}\right\}_{k=1}^{n}$ независимых с. в. таких, что $\lambda\left\{g_{k}=1\right\}=1 / r$ и $\lambda\left\{g_{k}=0\right\}=(r-1) / r(k \geqslant 1)$, где $r \in \mathbb{N}$ и $1<r \leqslant n$. Предположим также, что эта последовательность независима относительно последовательности $\left\{f_{k}\right\}_{k=1}^{n}$. Будем считать, что все функции определены на квадрате $[0,1] \times[0,1], f_{k}=f_{k}(t), g_{k}=g_{k}(s)$. Рассмотрим последовательность $\left\{f_{k} g_{k}\right\}_{k=1}^{n}$, обозначив через $\mathscr{A}$ и $\mathscr{B}$ соответствующие ей величины, аналогичные $A$ и $B$ 
(определенным ранее для последовательности $\left\{f_{k}\right\}_{k=1}^{n}$ ). Применяя к ней (62), получим, что

$$
\frac{1}{2} \int_{0}^{1} \mathscr{A}(u) d u \leqslant \int_{0}^{1} \int_{0}^{1} \mathscr{B}(s, t) d s d t \leqslant \int_{0}^{1} \mathscr{A}(u) d u .
$$

Так как $\left(f_{k} g_{k}\right)^{*}=\left(\sigma_{1 / r} f_{k}\right)^{*}$ (см. доказательство теоремы 22), где, как обычно, $\sigma_{1 / r} f_{k}(t)=f_{k}(r t)$, то $\mathscr{A}(u)=\sigma_{1 / r} A(u)(u>0)$. Следовательно,

$$
\int_{0}^{1} \mathscr{A}(u) d u=\int_{0}^{1} \sigma_{1 / r} A(u) d u=\int_{0}^{1} A(r u) d u=\frac{1}{r}\left(\int_{0}^{1} A(u) d u+\int_{1}^{r} A(u) d u\right),
$$

и ввиду неравенства $\sum_{k=2}^{r} A(k) \leqslant \int_{1}^{r} A(u) d u \leqslant \sum_{k=1}^{r} A(k)$ из предыдущих следует:
шенино-

$$
\begin{aligned}
\frac{1}{4 r}\left(\int_{0}^{1} A(u) d u+\sum_{k=1}^{r} A(k)\right) & \leqslant \int_{0}^{1} \int_{0}^{1} \mathscr{B}(s, t) d s d t \\
& \leqslant \frac{1}{r}\left(\int_{0}^{1} A(u) d u+\sum_{k=1}^{r} A(k)\right) .
\end{aligned}
$$

Покажем, что из последнего неравенства вытекает (61) в случае $X=L_{1}$, $E=\mathbf{s}_{\mathbf{r}}$. Рассмотрим оператор $\mathrm{E}_{\mathscr{M}}$ условного математического ожидания относительно $\sigma$-подалгебры $\mathscr{M} \sigma$-алгебры измеримых по Лебегу подмножеств квадрата $[0,1] \times[0,1]$, состоящей из всех множеств вида $e \times[0,1]$, где $e-$ произвольное измеримое подмножество $[0,1]$. Если $f$ суммируема на $[0,1] \times[0,1]$, то $\mathrm{E}_{\mathscr{M}} f(t)=\int_{0}^{1} f(t, s) d s$. В частности,

$$
\mathrm{E}_{\mathscr{M}} \mathscr{B}(t)=\int_{0}^{1} \max _{1 \leqslant k \leqslant n} f_{k}(t) g_{k}(s) d s .
$$

Если теперь $\mathscr{A}_{t}$ - величина, аналогичная $A$ и соответствующая последовательности $\left\{f_{k}(t) g_{k}(\cdot)\right\}_{k=1}^{n}$, то для каждого $t \in[0,1]$

$$
\int_{0}^{1} \mathscr{A}_{t}(u) d u=\int_{0}^{1} \sum_{k=1}^{r}\left(f_{k}(t)\right)^{\sharp} \chi_{((k-1) / r, k / r)}(s) d s=\frac{1}{r} \sum_{k=1}^{r}\left(f_{k}(t)\right)^{\sharp} .
$$

Следовательно, применяя (62) к последовательности $\left\{f_{k}(t) g_{k}(\cdot)\right\}_{k=1}^{n}$, получаем

$$
\frac{1}{2 r} \sum_{k=1}^{r}\left(f_{k}(t)\right)^{\sharp} \leqslant \mathrm{E}_{\mathscr{M}} \mathscr{B}(t) \leqslant \frac{1}{r} \sum_{k=1}^{r}\left(f_{k}(t)\right)^{\sharp}, \quad 0<t \leqslant 1 .
$$

Так как $\int_{0}^{1} \int_{0}^{1} \mathscr{B}(s, t) d s d t=\int_{0}^{1} \mathrm{E}_{\mathscr{M}} \mathscr{B}(t) d t$, то из (64) и (63) следует, что

$$
\frac{1}{4}\left(\int_{0}^{1} A(u) d u+\sum_{k=1}^{r} A(k)\right) \leqslant \int_{0}^{1} \sum_{k=1}^{r}\left(f_{k}(t)\right)^{\sharp} d t \leqslant 2\left(\int_{0}^{1} A(u) d u+\sum_{k=1}^{r} A(k)\right),
$$

и неравенство (61) доказано, если $X=L_{1}$, а $E=\mathbf{s}_{r}(r=1,2, \ldots)$. 
Рассмотрим общий случай. Прежде всего, заметим, что неравенство (61) будет следствием оценок

$$
\left\|A \chi_{[0,1]}\right\|_{X} \leqslant 2\|\| \sum_{k=1}^{n} f_{k} e_{k}\left\|_{E}\right\|_{X} \text { и }\left\|\sum_{k=1}^{n} A(k) e_{k}\right\|_{E} \leqslant 4\|\| \sum_{k=1}^{n} f_{k} e_{k}\left\|_{E}\right\|_{X} .
$$

Первая из них ввиду очевидного соотношения $\max _{1 \leqslant k \leqslant n} f_{k} \leqslant\left\|\sum_{k=1}^{n} f_{k} e_{k}\right\|_{E}$, а также предложения 1 сразу вытекает из левой части неравенства, доказанного в предложении 7.

Для доказательства второго из неравенств (66) воспользуемся двойственностью. Прежде всего,

$$
\begin{aligned}
&\left\|\sum_{k=1}^{n} f_{k} e_{k}\right\|_{E}=\sup _{b=\left\{b_{k}\right\}_{k=1}^{n},\|b\|_{E} \times} \leqslant 1 \\
&=\sum_{k=1}^{n}\left(f_{k}(t)\right)^{\sharp} b_{k}^{\sharp} \sup _{k=1}^{n},\|b\|_{E \times} \leqslant 1 \\
& \sum_{r=1}^{n}\left\|\left(f_{k}(t)\right)_{k=1}^{n}\right\|_{\mathbf{s}_{r}}\left(b_{r}^{\sharp}-b_{r+1}^{\sharp}\right), \quad b_{n+1}^{\sharp}=0,
\end{aligned}
$$

где двойственное пространство $E^{\times}$определяется совершенно аналогично тому, как это делается в случае функциональных пространств. Поэтому

$$
\int_{0}^{1}\left\|\sum_{k=1}^{n} f_{k}(t) e_{k}\right\|_{E} d t \geqslant \sup _{b=\left\{b_{k}\right\}_{k=1}^{n},\|b\|_{E} \times \leqslant 1} \sum_{r=1}^{n}\left(b_{r}^{\sharp}-b_{r+1}^{\sharp}\right) \int_{0}^{1}\left\|\left(f_{k}(t)\right)_{k=1}^{n}\right\|_{\mathbf{s}_{r}} d t .
$$

Отсюда и из (65) следует, что

$$
\begin{aligned}
4 \int_{0}^{1}\left\|\sum_{k=1}^{n} f_{k}(t) e_{k}\right\|_{E} d t & \geqslant \sup _{b=\left\{b_{k}\right\}_{k=1}^{n},\|b\|_{E} \times \leqslant 1} \sum_{r=1}^{n}\left(b_{r}^{\sharp}-b_{r+1}^{\sharp}\right) \sum_{k=1}^{r} A(k) \\
& =\sup _{b=\left\{b_{k}\right\}_{k=1}^{n},\|b\|_{E} \times \leqslant 1} \sum_{k=1}^{n} A(k) b_{k}^{\sharp}=\left\|\sum_{k=1}^{n} A(k) e_{k}\right\|_{E} .
\end{aligned}
$$

Так как для произвольного симметричного пространства $X$ на $[0,1]$ выполнено неравенство $\|f\|_{L_{1}} \leqslant\|f\|_{X}(f \in X)$, то получаем нужное нам неравенство, и теорема доказана.

Ключевой момент доказательства последней теоремы состоит в применении двойственности, и поэтому оно не может быть обобщено на квазинормированные симметричные пространства последовательностей. Кроме того, в его заключительной части используется вложение $X \subset L_{1}$, вообще говоря, неверное для квазинормированных симметричных пространств $X$. В то же время, как показано в недавней работе [60], справедливость неравенства (61) обусловлена исключительно вероятностно-комбинаторными причинами. Приведем без доказательства некоторые результаты, полученные в [60].

ТеОрема 27. Пусть $n \in \mathbb{N} u\left\{f_{k}\right\}_{k=1}^{n}-$ произвольная последовательность неотрицательных независимых функиий на $[0,1]$. Тогда

$$
\lambda\left\{t \in[0,1]: \sum_{k=1}^{n} f_{k}(t) \geqslant \frac{1}{2} \sum_{k=1}^{n} \mathbf{f}^{*}(k)\right\} \geqslant \frac{1}{8} .
$$


СлеДСТвИЕ 4. Пусть $X$ - произвольное квазинормированное симметричное пространство на $[0,1]$. Тогда существует константа $\alpha>0$, зависящая лишь от $X$, такая, что для каждого $n \in \mathbb{N}$ и любой последовательности неотрицательных независимых функций $\left\{f_{k}\right\}_{k=1}^{n}$ на $[0,1]$ справедливо неравенство

$$
\left\|\mathbf{f}^{*} \chi_{[0,1]}\right\|_{X}+\sum_{k=1}^{n} \mathbf{f}^{*}(k) \leqslant \alpha\left\|\sum_{k=1}^{n} f_{k}\right\|_{X} .
$$

ТеОрема 28. Пусть $n \in \mathbb{N} u\left\{f_{k}\right\}_{k=1}^{n}-$ произвольная последовательность равноизмеримых неотрицательных независимых функций на $[0,1]$. Тогда существует константа $c_{0}>0$, не зависящая от $n$ и функций $f_{k}$, для которой въполнено:

$$
\lambda\left\{t \in[0,1]: f_{j}^{\sharp}(t) \geqslant \mathbf{f}^{*}(2 j-1), j=1, \ldots,\left[\frac{n}{2}\right]\right\} \geqslant c_{0} .
$$

СлеДСТвИЕ 5. Пусть Х и Е-произволъные квазинормированные симметричные пространства функиий на $[0,1]$ и последовательностей соответственно, $\left\{e_{k}\right\}_{k=1}^{\infty}-$ стандартный базис в $E$. Тогда существует константа $C>0$, зависящая лищь от константы из неравенства треугольника в $E$, такая, что для любого $n \in \mathbb{N}$ и всякой последовательности равноизмеримых неотрицательных независимых функиий $\left\{f_{k}\right\}_{k=1}^{n} \subset X$ выполнено неравенство

$$
\left\|\sum_{k=1}^{n} \mathbf{f}^{*}(k) e_{k}\right\|_{E} \leqslant C\|\| \sum_{k=1}^{n} f_{k} e_{k}\left\|_{E}\right\|_{X},
$$

6.2. Верхние оценки в симметричных пространствах со свойством Круглова. Рассмотрим теперь противоположное по отношению к (61) неравенство

$$
\|\| \sum_{k=1}^{n} f_{k} e_{k}\left\|_{E}\right\|_{X} \leqslant C\left(\left\|\mathbf{f}^{*} \chi_{[0,1]}\right\|_{X}+\left\|\sum_{k=1}^{n} \mathbf{f}^{*}(k) e_{k}\right\|_{E}\right),
$$

где, как и ранее, $\mathbf{f}$ - дизъюнктная сумма последовательности $\left\{f_{k}\right\}_{k=1}^{n} \subset X$.

Если $E=\ell_{1}$ и $X=L_{p}(1 \leqslant p<\infty)$, то это соотношение совпадает (с точностью до константы) с правой частью неравенства Розенталя (6). В более общей ситуации, когда $E=\ell_{1}$, а $X$ - произвольное симметричное пространство, содержащее пространство $L_{p}$ при некотором $p<\infty$, мы получаем неравенство (27), доказанное У. Джонсоном и Г. Шехтманом [35]. Позднее С. Монтгомери-Смит [57; теорема 1] распространил (67) на произвольные симметричные пространства последовательностей $E$ (с тем же условием на $X$ ). Во всех упомянутых работах доказательство в решающей степени было основано на применении хорошо известного неравенства Хоффмана-Йоргенсона [36] или его вариантов. В [42; теорема 6.7], напротив, была использована конструкция оператора Круглова и интерполяция операторов. Оценка (67) была получена там для любого симметричного пространства $X$ со свойством Круглова, но лишь в специальном случае, когда $E=\ell_{q}(1 \leqslant q \leqslant \infty)$. Здесь мы докажем наиболее общий (и фактически окончательный) результат такого рода из более 
поздней работы [61; теорема 1], который верен не только для нормированных, но и для квазинормированных симметричных пространств последовательностей, удовлетворяющих некоторым не очень ограничительным условиям.

В дальнейшем нам понадобится неравенство (67) в частном случае $E=\ell_{q}$ $(1 \leqslant q \leqslant \infty)$ с точной по порядку константой при $q \rightarrow \infty$. Как уже говорилось, его первоначальная версия была получена в работе [42]; здесь мы приводим более точный результат из [44], в котором выявлена зависимость константы $C$ от нормы оператора Круглова и $q$ (что будет играть решающую роль в доказательстве следующей теоремы).

Теорема 29. Пусть $X$ и $Y$-симметричные пространства на $[0,1], X \subseteq Y$ с константой 1 и норма в $Y$ порядково полунепрерьвна. Если оператор $\mathscr{K}$ ограниченно действует из $X$ в $Y$, то существует такая универсальная константа $\beta>0$, что для всех $n \in \mathbb{N}$, произвольной последовательности независимых функиий $\left\{f_{k}\right\}_{k=1}^{n} \subset X$ и любого $q \in[1, \infty]$ справедливо неравенство

$$
\|\|\left(f_{k}\right)_{k=1}^{n}\left\|_{\ell_{q}}\right\|_{Y} \leqslant \beta\|\mathscr{K}\|_{X \rightarrow Y}^{1 / q}\left(\left\|\mathbf{f}^{*} \chi_{[0,1]}\right\|_{X}+\left\|\left(\mathbf{f}^{*}(k)\right)_{k=1}^{n}\right\|_{\ell_{q}}\right) .
$$

В доказательстве нам понадобится следующая конструкция из теории банаховых пространств [17; с. 46]. Если $X$ - симметричное пространство на $[0,1]$, то $\widehat{X\left(\ell_{p}\right)}-$ множество всех таких последовательностей $\left\{f_{k}\right\}_{k=1}^{\infty} \subset X$, что

$$
\|\mathbf{f}\|_{\widetilde{X\left(\ell_{p}\right)}}:=\sup _{n=1,2, \ldots}\left\|\left(\sum_{k=1}^{n}\left|f_{k}\right|^{p}\right)^{1 / p}\right\|_{X}<\infty
$$

(с естественной модификацией при $p=\infty$ ). Замкнутое подпространство пространства $\widehat{X\left(\ell_{p}\right)}$, порожденное всеми финитными последовательностями $\left\{f_{k}\right\} \in$ $\widetilde{X\left(\ell_{p}\right)}$, будем обозначать через $X\left(\ell_{p}\right)$.

ДоКАЗАТЕЛЬСТВо тЕОРЕмЫ 29. Предположим сначала, что пространство $Y$ максимально. Тогда, как нетрудно проверить, утверждение (ii) теоремы 22 справедливо для бесконечных сумм, и поэтому с некоторой универсальной константой $\alpha \geqslant 1$

$$
\left\|\left(f_{k}\right)_{k=1}^{\infty}\right\|_{Y\left(\ell_{1}\right)}:=\left\|\sum_{k=1}^{\infty}\left|f_{k}\right|\right\|_{Y} \leqslant \alpha\|\mathscr{K}\|_{X \rightarrow Y}\|\mathbf{f}\|_{Z_{X}^{1}} .
$$

Кроме того, ввиду условия теоремы и предложения 7

$$
\left\|\left(f_{k}\right)\right\|_{Y\left(\ell_{\infty}\right)} \leqslant\left\|\left(f_{k}\right)\right\|_{X\left(l_{\infty}\right)} \leqslant\|\mathbf{f}\|_{Z_{X}^{\infty}}
$$

Далее мы используем интерполяционные свойства конструкции Кальдерона (см. раздел 2). Пусть $(\Omega, \mathscr{P}):=\prod_{k=0}^{\infty}\left([0,1], \lambda_{k}\right)$ и $\delta:(\Omega, \mathscr{P}) \rightarrow([0,1], \lambda)$ - отображение, сохраняющее меру. Для каждой функции $g \in S(\Omega, \mathscr{P})$ положим $T g(x):=g\left(\delta^{-1} x\right) \quad(x \in[0,1])$. Тогда отображение $T: S(\Omega, \mathscr{P}) \rightarrow S([0,1], \lambda)$ 
переводит каждую функцию $g$ в равноизмеримую ей, т. е. $(T g)^{*}=g^{*}$. Определим, наконец, положительный линейный оператор $Q$ из $S(0, \infty)$ в $S(\Omega, \mathscr{P})$ следующим образом:

$$
Q f\left(\omega_{0}, \omega_{1}, \ldots\right):=\left\{f_{k}\left(\omega_{k}\right)\right\}_{k=0}^{\infty}, \quad f \in S(0, \infty),
$$

где $f_{k}\left(\omega_{k}\right):=f\left(\omega_{k}+k\right)\left(\omega_{k} \in[0,1]\right), k \geqslant 0$. Аргументы, приведенные в начале доказательства, показывают, что положительный оператор $Q^{\prime}:=T Q$ ограничен из $Z_{X}^{1}$ в $Y\left(\ell_{1}\right)$ и из $Z_{X}^{\infty}$ в $Y\left(\ell_{\infty}\right)$ с нормами, не превосходящими $\alpha\|\mathscr{K}\|_{X \rightarrow Y}$ и 1 соответственно. Поэтому

$$
Q^{\prime}:\left(Z_{X}^{1}\right)^{1-\theta}\left(Z_{X}^{\infty}\right)^{\theta} \rightarrow\left(Y\left(\ell_{1}\right)\right)^{1-\theta}\left(Y\left(\ell_{\infty}\right)\right)^{\theta}
$$

и

$$
\left\|Q^{\prime}\right\| \leqslant\left\|Q^{\prime}\right\|_{Z_{X}^{1} \rightarrow Y\left(\ell_{1}\right)}^{1-\theta}\left\|Q^{\prime}\right\|_{Z_{X}^{\infty} \rightarrow Y\left(\ell_{\infty}\right)}^{\theta} \leqslant \alpha^{1-\theta}\|\mathscr{K}\|_{X \rightarrow Y}^{1-\theta}, \quad \theta \in(0,1) .
$$

Если $q=1 /(1-\theta)$, то для каждого $\theta \in(0,1)$ имеют место вложения

$$
Z_{X}^{q} \subseteq\left(Z_{X}^{1}\right)^{1-\theta}\left(Z_{X}^{\infty}\right)^{\theta}, \quad\left(Y\left(\ell_{1}\right)\right)^{1-\theta}\left(Y\left(\ell_{\infty}\right)\right)^{\theta} \subseteq Y\left(\ell_{q}\right)
$$

В самом деле, чтобы проверить первое из них, для произвольной $g \in Z_{X}^{q}$ такой, что $g=g^{*}$ и $\|g\|_{Z_{X}^{q}}=1$, положим

$$
g_{1}:=g \chi_{[0,1]}+g^{q} \chi_{[1, \infty)}, \quad g_{\infty}:=g \chi_{[0,1]}+\chi_{[1, \infty)} .
$$

Очевидно, что $g=\left(g_{1}\right)^{1-\theta}\left(g_{\infty}\right)^{\theta}$. Кроме того, так как $g(1) \leqslant 1$, то $g_{1}$ убывает. Следовательно, $g_{i} \in Z_{X}^{i}$ и $\left\|g_{i}\right\|_{Z_{X}^{i}} \leqslant 2(i=1, \infty)$. Второе вложение (и даже равенство) доказано в [62; теорема 3$]$. В итоге мы получаем, что с некоторой универсальной константой $\gamma>0$

$$
\left\|Q^{\prime}\right\|_{Z_{X}^{q} \rightarrow Y\left(\ell_{q}\right)} \leqslant \alpha^{1 / q}\|\mathscr{K}\|_{X \rightarrow Y}^{1 / q} \gamma \leqslant \max \{\alpha, \gamma\}\|\mathscr{K}\|_{X \rightarrow Y}^{1 / q} .
$$

Утверждение теоремы теперь вытекает из следующего легко проверяемого неравенства

$$
\|\mathbf{f}\|_{Z_{X}^{q}} \leqslant\left\|\mathbf{f}^{*} \chi_{[0,1]}\right\|_{X}+\left\|\left(\mathbf{f}^{*}(k)\right)\right\|_{\ell_{q}} \leqslant 2\|\mathbf{f}\|_{Z_{X}^{q}}
$$

справедливого для произвольного симметричного пространства $X$ и всех $1 \leqslant$ $q \leqslant \infty$.

Пусть теперь мы знаем лишь то, что норма $Y$ порядково полунепрерывна. Тогда $Y$ изометрически вложено в максимальное пространство $Y^{\times \times}$(см. раздел 2). Поэтому результат следует из уже рассмотренного случая и того, что $X \subseteq Y$.

ЗАмечАнИЕ 7 . Пусть $X$ - симметричное пространство на $[0,1]$, для которого существует такая константа $C=C_{X}>0$, что для каждой последовательности независимых функций $\left\{f_{k}\right\}_{k=1}^{n} \subset X(n \in \mathbb{N})$ выполнено неравенство (67). Как уже говорилось в п. 4.1, в классическом случае $X=L_{p}(1 \leqslant p<\infty)$ это неравенство Розенталя. В доказательствах самого Розенталя (см. теорему 6), а также Буркхольдера в [63] несколько более общего результата константа $C_{L_{p}}$ в (67) экспоненциально растет при $p \rightarrow \infty$. Точная (по порядку) константа 
$C_{L_{p}} \asymp p / \ln (p+1) \quad(1 \leqslant p<\infty)$ была найдена в работе [64] (см. также альтернативные доказательства в [31] и [58]). Понятие оператора Круглова позволяет рассмотреть этот вопрос с более общих позиций. Из теорем 22 и 23 вытекает, что наилучшая константа $C_{X}$ в неравенстве $(67)$ в случае сепарабельных или максимальных симметричных пространств должна быть эквивалентна норме оператора $\mathscr{K}$ в $X$. Благодаря этому в работе [44] найдены точные (по порядку) константы в неравенстве (67) для различных шкал симметричных пространств, в частности, для пространств Лоренца и Марцинкевича.

Теперь мы докажем аналогичное утверждение для общих квазибанаховых симметричных пространств последовательностей $E$, удовлетворяющих некоторым условиям. Первое условие состоит в том, что для каждого $k \in \mathbb{N}$ оператор растяжения

$$
\sigma_{k}\left(\left\{a_{j}\right\}_{j=1}^{\infty}\right):=(\underbrace{a_{1}, \ldots, a_{1}}_{k}, \underbrace{a_{2}, \ldots, a_{2}}_{k}, \ldots, \underbrace{a_{j}, \ldots, a_{j}}_{k}, \ldots)
$$

ограничен в $E$ и справедливо неравенство

$$
\text { (i) }\left\|\sigma_{k}\right\|_{E \rightarrow E} \leqslant k, \quad k \in \mathbb{N} \text {. }
$$

Второе условие заключается в выполнении непрерывного вложения

$$
\text { (ii) } \ell_{1} \subseteq E \text {. }
$$

Заметим, что (банахово) симметричное пространство последовательностей $E$ очевидным образом удовлетворяет как условию (i), так и условию (ii).

ТЕорема 30. Предположим, что функциональное симметричное пространство $X$ с порядково полунепрерывной нормой имеет свойство Круглова, а квазибанахово симметричное пространство последовательностей Е удовлетворяет условиям (i) $и$ (ii). Тогда существует константа $C>0$, зависящая только от $X$, такал, что для всех $n \in \mathbb{N}$ и произвольной последовательности независимых функциц $\left\{f_{k}\right\}_{k=1}^{n} \subset X$ выполнено неравенство (67).

ДокАЗАтЕльство. Ключевую роль в нашем подходе играет "слабое" $\ell_{1}$-пространство, $\ell_{1, \infty}$ (см. также [58; доказательство теоремы 0.2]). Оно состоит из всех последовательностей $a=\left(a_{k}\right)_{k=1}^{\infty}$, для которых

$$
\|a\|_{1, \infty}:=\sup _{k \geqslant 1} k a_{k}^{*}<\infty .
$$

Легко видеть, что $\ell_{1, \infty}$ - квазибанахово симметричное пространство последовательностей и что

$$
\|a+b\|_{1, \infty} \leqslant 2\left(\|a\|_{1, \infty}+\|b\|_{1, \infty}\right) \quad\left(a, b \in \ell_{1, \infty}\right) .
$$

Сначала мы докажем неравенство (67) в этом специальном случае.

ПРЕДЛОЖЕНИЕ 8. Если Функииональное симметричное пространство $X$ имеет свойство Круглова, то существует такая универсальная константа 
$C_{0}>0$, что для всех $n \in \mathbb{N}$ и произвольной последовательности независимьх функицй $\left\{f_{k}\right\}_{k=1}^{n} \subset X$

$$
\|\| \sum_{k=1}^{n} f_{k} e_{k}\left\|_{\ell_{1, \infty}}\right\|_{X} \leqslant C_{0}\left(\left\|\mathbf{f}^{*} \chi_{[0,1]}\right\|_{X}+\left\|\sum_{k=1}^{n} \mathbf{f}^{*}(k) e_{k}\right\|_{\ell_{1, \infty}}\right) .
$$

ДокАзАтельство. Так как доказательство достаточно длинно, приведем здесь лишь его ключевые моменты (подробнее см. в [61]).

Не ограничивая общности, мы можем (и будем) предполагать, что последовательность $\left\{f_{k}\right\}_{k=1}^{n} \subset X$ состоит из неотрицательных функций. Положим $t_{0}:=\mathbf{f}^{*}(1)$ и заметим, что

$$
\sum_{k=1}^{n} \lambda\left\{s \in[0,1]: f_{k}(s)>t_{0}\right\}=\lambda\left\{s>0: \mathbf{f}(s)>t_{0}\right\} \leqslant 1 .
$$

Следовательно, если $g_{k}:=f_{k} \chi_{\left\{f_{k}>t_{0}\right\}}$ и $h_{k}:=f_{k} \chi_{\left\{f_{k} \leqslant t_{0}\right\}}(k=1, \ldots, n)$, то $f_{k}=g_{k}+h_{k}$ и $\sum_{k=1}^{n} \lambda\left(\operatorname{supp} g_{k}\right) \leqslant 1$. Оценим величины

$$
\|\| \sum_{k=1}^{n} g_{k} e_{k}\left\|_{\ell_{1, \infty}}\right\|_{X} \text { и }\|\| \sum_{k=1}^{n} h_{k} e_{k}\left\|_{\ell_{1, \infty}}\right\|_{X}
$$

по отдельности. Пусть $\mathbf{g}$ и $\mathbf{h}$ - дизъюнктные суммы последовательностей $\left\{g_{k}\right\}_{k=1}^{n}$ и $\left\{h_{k}\right\}_{k=1}^{n}$ соответственно. Тогда из определения функций $g_{k}$ и $h_{k}$ следует, что $\mathbf{g}^{*}=\mathbf{g}^{*} \chi_{[0,1]}=\mathbf{f}^{*} \chi_{[0,1]}$. Так как $\|a\|_{\ell_{1, \infty}} \leqslant\|a\|_{\ell_{1}}\left(a \in \ell_{1}\right)$, то по теореме 22

$$
\|\| \sum_{k=1}^{n} g_{k} e_{k}\left\|_{\ell_{1, \infty}}\right\|_{X} \leqslant\left\|\sum_{k=1}^{n} g_{k}\right\|_{X} \leqslant 8\|\mathscr{K}\|_{X \rightarrow X}\left\|\mathbf{g}^{*}\right\|_{X}=8\|\mathscr{K}\|_{X \rightarrow X}\left\|\mathbf{f}^{*} \chi_{[0,1]}\right\|_{X} .
$$

Осталось доказать аналогичную оценку для последовательности $\left\{h_{k}\right\}_{k=1}^{n}$, состоящей из равномерно ограниченных независимых функций. Прежде всего, с помощью теоремы 29 в случае $X=L_{p}(p \geqslant 1)$ можно показать, что для некоторой универсальной константы $\gamma>0$

$$
\|\| \sum_{k=1}^{n} h_{k}(u) e_{k}\left\|_{\ell_{1, \infty}}\right\|_{p} \leqslant \gamma\left\|\sum_{k=1}^{n} \mathbf{f}^{*}(k) e_{k}\right\|_{\ell_{1, \infty}} \frac{p}{\sqrt{\ln (p+1)}}, \quad p \geqslant 1,
$$

откуда

$$
\sup _{p \geqslant 1}\left(\frac{\sqrt{\ln (p+1)}}{p}\|\| \sum_{k=1}^{n} h_{k}(u) e_{k}\left\|_{\ell_{1, \infty}}\right\|_{p}\right) \leqslant \gamma\left\|\sum_{k=1}^{n} \mathbf{f}^{*}(k) e_{k}\right\|_{\ell_{1, \infty}} .
$$

Величина из левой части этого неравенства эквивалентна норме функции

$$
u \mapsto\left\|\sum_{k=1}^{n} h_{k}(u) e_{k}\right\|_{\ell_{1, \infty}}
$$


в уже знакомом нам пространстве Орлича $L_{M_{2}}$, где $M_{2}(u)=e^{u \sqrt{\ln (u+e)}}-1$ (см., например, [65; следствие 1] или [64; предложение 3.6]). Так как $X$ имеет свойство Круглова, то из теоремы 14 следует, что $L_{M_{2}} \subseteq X$. Поэтому для некоторой универсальной константы $\gamma^{\prime}>0$ получаем

$$
\|\| \sum_{k=1}^{n} h_{k}(u) e_{k}\left\|_{\ell_{1, \infty}}\right\|_{X} \leqslant \gamma^{\prime}\left\|\sum_{k=1}^{n} \mathbf{f}^{*}(k) e_{k}\right\|_{\ell_{1, \infty}} .
$$

Это вместе с оценкой (71) и неравенством (69) дает нам (70), и доказательство предложения закончено.

Прежде чем продолжить доказательство теоремы 30, введем некоторые дополнительные обозначения. Пусть $E$ - квазибанахово симметричное пространство последовательностей. Для данного $n \in \mathbb{N}$ определим оператор $U_{n}^{E}$, действующий из множества всех квадратных матриц размера $n \times n$ в $n^{n}$-мерное пространство ступенчатых функций в $L_{1}(0,1)$, порожденное характеристическими функциями промежутков $\Delta_{i}:=\left[(i-1) n^{-n}, i n^{-n}\right), i=1, \ldots, n^{n}$. Чтобы задать этот оператор, рассмотрим множество $\{1, \ldots, n\}^{n}$ и произвольное взаимно однозначное отображение $\phi$ этого множества в множество промежутков $\left\{\Delta_{i}\right\}_{i=1}^{n^{n}}$. Для каждой матрицы $\alpha=\left(\alpha_{i, j}\right)_{i, j=1}^{n}$ ее образом $U_{n}^{E}(\alpha)$ является функция

$$
U_{n}^{E} \alpha(t):=\sum_{j_{1}, \ldots, j_{n}=1}^{n}\left\|\sum_{k=1}^{n} \alpha_{k, j_{k}} e_{k}\right\|_{E} \chi_{\Delta_{\phi\left(j_{1}, \ldots, j_{n}\right)}}(t) \quad(0 \leqslant t \leqslant 1) .
$$

Определение оператора $U_{n}^{E}$, разумеется, зависит от выбора отображения $\phi$. Тем не менее, если $X$ - симметричное пространство на $[0,1]$, то норма $\left\|U_{n}^{E}(\alpha)\right\|_{X}$ от $\phi$ не зависит.

Далее, напомним, что матрица $\mu=\left(\mu_{i, j}\right)_{i, j=1}^{n}$ называется бистохастической, если

$$
\mu_{i, j} \geqslant 0, \quad \sum_{i=1}^{n} \mu_{i, j}=\sum_{j=1}^{n} \mu_{i, j}=1, \quad 1 \leqslant i, j \leqslant n .
$$

Для каждой такой матрицы определим семейство $\xi^{\mu}=\left\{\xi_{k}^{\mu}\right\}_{k=1}^{n}$ независимых с. в., заданных на вероятностном пространстве $[0,1]^{n}$ следующим образом. Если $k=1, \ldots, n, i=1, \ldots, n$ и $t=\left(t_{1}, \ldots, t_{n}\right) \in[0,1]^{n}$, то $\xi_{k}^{\mu}(t)=\xi_{k}^{\mu}\left(t_{k}\right)=1 / i$ на некотором подмножестве $[0,1]$, мера которого равна $\mu_{i, k}$. Так как матрица $\mu$ бистохастическая, то

$$
\sum_{k=1}^{n} \lambda\left\{\xi_{k}^{\mu}=\frac{1}{i}\right\}=1 \quad(i=1, \ldots, n) .
$$

Кроме того, если для $t \in[0,1]^{n}$ мы имеем $\xi_{k}^{\mu}(t)=1 / i_{k}$, где $1 \leqslant i_{k} \leqslant n(1 \leqslant$ $k \leqslant n)$, то

$$
\begin{aligned}
\left\|\xi_{k}^{\mu}(t)\right\|_{l_{1, \infty}} & =\sup _{k=1, \ldots, n} k \xi_{k}^{\mu}(t)^{*}=\sup _{r=1, \ldots, n} \frac{1}{r} \operatorname{card}\left\{j: \xi_{j}^{\mu}(t) \geqslant \frac{1}{r}\right\} \\
& =\sup _{r=1, \ldots, n} \frac{1}{r} \operatorname{card}\left\{k: j_{k} \leqslant r\right\} .
\end{aligned}
$$


В этих обозначениях мы можем следующим образом сформулировать результат, неявно содержащийся в доказательстве предложения 2.1 работы [59].

ПРеДЛОЖеНИЕ 9. Предположим, что X - произволъное функционалъное симметричное пространство, а $Е$ - квазибанахово симметричное пространство последовательностей, удовлетворяющее условию (i) (с. 57). Тогда для каждой матрицы $\alpha=\left(\alpha_{i, j}\right)_{i, j=1}^{n}$ существует такая бистохастическая матрича $\mu=\left(\mu_{i, j}\right)_{i, j=1}^{n}$, что

$$
\left\|U_{n}^{E} \alpha\right\|_{X} \leqslant 2\left\|\max \left\{1,\left\|\xi^{\mu}\right\|_{1, \infty}\right\}\right\|_{X}\left\|\sum_{k=1}^{n} \alpha_{(k-1) n+1}^{*} e_{k}\right\|_{E},
$$

где через $\left\{\alpha_{j}^{*}\right\}_{j=1}^{n^{2}}$ обозначается невозрастающая перестановка последовательности $\left\{\left|\alpha_{i, j}\right|\right\}_{i, j=1}^{n}$.

Применим теперь предложения 8 и 9 к доказательству теоремы 30. Так как $X$ имеет свойство Круглова, то ввиду предложения 8

$$
\|\| \xi^{\mu}\left\|_{1, \infty}\right\|_{X} \leqslant C_{0}\left(\left\|\mathbf{f}_{\xi}^{*} \chi_{[0,1]}\right\|_{X}+\left\|\sum_{k=1}^{n} \mathbf{f}_{\xi}^{*}(k) e_{k}\right\|_{\ell_{1, \infty}}\right)
$$

где $\mathbf{f}_{\xi}$ - дизъюнктная сумма последовательности $\xi^{\mu}=\left\{\xi_{k}^{\mu}\right\}_{k=1}^{n}$. Так как из (72) немедленно следует, что

$$
\mathbf{f}_{\xi}^{*}(u)=\sum_{i=1}^{n} \frac{1}{i} \chi_{[i-1, i)}(u)
$$

TO

$$
\left\|\mathbf{f}_{\xi}^{*} \chi_{[0,1]}\right\|_{X} \leqslant 1 \quad \text { и }\left\|\sum_{k=1}^{n} \mathbf{f}_{\xi}^{*}(k) e_{k}\right\|_{1, \infty} \leqslant 1 .
$$

Тем самым \|\|$\xi^{\mu}\left\|_{1, \infty}\right\|_{X} \leqslant 2 C_{0}$, и, применяя предложение 9, мы получаем следующее весьма общее утверждение, которое можно рассматривать как аналог хорошо известных результатов Квапеня-Шютта [6].

СлЕДСТвИЕ 6. Если функииональное симметричное пространство $X$ имеет свойство Круглова, то для каждого $n \in \mathbb{N}$, для произвольного квазибанахова симметричного пространства последовательностей $E$, удовлетворяющего условию (i), и для любой матрицы $\alpha=\left(\alpha_{i, j}\right)_{i, j=1}^{n}$ справедливо неравенство

$$
\left\|U_{n}^{E} \alpha\right\|_{X} \leqslant K_{0}\left\|\sum_{k=1}^{n} \alpha_{(k-1) n+1}^{*} e_{k}\right\|_{E}
$$

где константа $K_{0}$ зависит только от $X$.

Теперь мы готовы к тому, чтобы закончить доказательство теоремы 30. Так как пространство $E$ симметрично, а функции $f_{k}(k=1, \ldots, n)$ независимы, мы можем (и будем) предполагать, что функции $f_{k}\left(s_{1}, \ldots, s_{n}\right)=f_{k}\left(s_{k}\right)$ определены на $[0,1]^{n}$, неотрицательны и не возрастают. Каждую из них представим в виде суммы трех дизъюнктных слагаемых следующим образом. Если $t_{0}=\mathbf{f}^{*}(1)$, то 
$f_{k}^{1}:=f_{k} \chi_{\left\{f_{k}>t_{0}\right\}}, f_{k}^{2}:=\left(f_{k}-f_{k}^{1}\right) \chi_{[0,1 / n)}$ и $f_{k}^{3}:=f-f_{k}^{1}-f_{k}^{2}$. Заметим, что $\sum_{k=1}^{n} \lambda\left(\operatorname{supp} f_{k}^{i}\right) \leqslant 1$, если $i=1,2$. Следовательно, ввиду условия (ii) (c. 57 ), а также по теореме 22

$$
\|\| \sum_{k=1}^{n} f_{k}^{i} e_{k}\left\|_{E}\right\|_{X} \leqslant\left\|\sum_{k=1}^{n} f_{k}^{i}\right\|_{X} \leqslant 8\|\mathscr{K}\|_{X \rightarrow X}\left\|\mathbf{f}^{*} \chi_{[0,1]}\right\|_{X} \quad(i=1,2) .
$$

Осталось показать, что аналогичная оценка справедлива и в случае $i=3$. Для этого применим следствие 6 . Как и в доказательстве теоремы 0.2 из [58], введем матрицу $\alpha=\left(\alpha_{i, j}\right)_{i, j=1}^{n}$, где

$$
\alpha_{i, j}:=\sup _{j / n<s_{i} \leqslant(j+1) / n} f_{i}^{3}\left(s_{i}\right), \quad j=1, \ldots, n-1, \quad \alpha_{i, n}=0 .
$$

Пусть $\left(j_{1}, \ldots, j_{n}\right) \in\{1, \ldots, n\}^{n}$. Тогда, если координаты вектора $s:=\left(s_{1}, \ldots, s_{n}\right)$ удовлетворяют неравенствам $j_{i} / n<s_{i} \leqslant\left(j_{i}+1\right) / n$, то

$$
\left\|\sum_{i=1}^{n} f_{i}^{3}\left(s_{i}\right) e_{i}\right\|_{E} \leqslant\left\|\sum_{i=1}^{n} \alpha_{i, j_{i}} e_{i}\right\|_{E}
$$

(заметим, что $f_{i}^{3}\left(s_{i}\right)=0$, если $\left.0 \leqslant s_{i} \leqslant 1 / n\right)$. Так как для любого $\left(j_{1}, \ldots, j_{n}\right) \in$ $\{1, \ldots, n\}^{n}$ выполнено равенство

$$
\lambda\left\{s \in[0,1]^{n}: \frac{j_{i}}{n}<s_{i} \leqslant \frac{j_{i}+1}{n}, i=1, \ldots, n\right\}=n^{-n},
$$

то ввиду следствия 6 заключаем:

$$
\|\| \sum_{k=1}^{n} f_{k}^{3} e_{k}\left\|_{E}\right\|_{X} \leqslant\left\|U_{n}^{E} \alpha\right\|_{X} \leqslant K_{0}\left\|\sum_{k=1}^{n} \alpha_{(k-1) n+1}^{*} e_{k}\right\|_{E} .
$$

Для оценки выражения из правой части неравенства (74) введем семейство независимых случайных величин $\left\{\tilde{f}_{i}\right\}_{i=1}^{n}$ на $[0,1]^{n}$, определяемых данной матрицей $\alpha=\left(\alpha_{i, j}\right)_{i, j=1}^{n}$ следующим образом:

$$
\tilde{f}_{i}(t)=\tilde{f}_{i}\left(t_{i}\right)=\sum_{j=1}^{n} \alpha_{i, j} \chi_{[(j-1) / n, j / n]}\left(t_{i}\right), \quad i=1, \ldots, n .
$$

Так как $\tilde{f}_{i}(t) \leqslant f_{i}(t)(i=1, \ldots, n)$, то для дизъюнктных сумм $\mathbf{f}(t):=\sum_{i=1}^{n} f_{i}(t-i+1) \chi_{(i-1, i]}(t) \quad$ и $\quad \tilde{\mathbf{f}}(t):=\sum_{i=1}^{n} \tilde{f}_{i}(t-i+1) \chi_{(i-1, i]}(t) \quad(t>0)$ выполнено: $\tilde{\mathbf{f}}(t) \leqslant \mathbf{f}(t)(t>0)$. Кроме того, $\tilde{\mathbf{f}}^{*}(k)=\alpha_{k n+1}^{*}(k=0,1, \ldots, n-1)$ и

$$
\alpha_{1}^{*}=\sup _{i=1, \ldots, n} \sup _{0<s_{i} \leqslant 1} f_{i}^{3}\left(s_{i}\right) \leqslant t_{0}=\mathbf{f}^{*}(1) .
$$


Следовательно, если $L$ - константа в неравенстве треугольника в пространстве $E$, то

$$
\begin{aligned}
\left\|\sum_{k=1}^{n} \alpha_{(k-1) n+1}^{*} e_{k}\right\|_{E} & \leqslant L\left(\alpha_{1}^{*}+\left\|\sum_{k=1}^{n-1} \alpha_{k n+1}^{*} e_{k}\right\|_{E}\right) \leqslant L\left(\mathbf{f}^{*}(1)+\left\|\sum_{k=1}^{n-1} \tilde{\mathbf{f}}^{*}(k) e_{k}\right\|_{E}\right) \\
& \leqslant L\left(\mathbf{f}^{*}(1)+\left\|\sum_{k=1}^{n-1} \mathbf{f}^{*}(k) e_{k}\right\|_{E}\right) \leqslant 2 L\left\|\sum_{k=1}^{n-1} \mathbf{f}^{*}(k) e_{k}\right\|_{E},
\end{aligned}
$$

откуда ввиду (74) находим, что

$$
\|\| \sum_{k=1}^{n} f_{k}^{3} e_{k}\left\|_{E}\right\|_{X} \leqslant 2 K_{0} L\left\|\sum_{k=1}^{n-1} \mathbf{f}^{*}(k) e_{k}\right\|_{E}
$$

Так как для всех $t \in[0,1]^{n}$

$$
\left\|\sum_{k=1}^{n} f_{k}(t) e_{k}\right\|_{E} \leqslant L^{2}\left(\left\|\sum_{k=1}^{n} f_{k}^{1}(t) e_{k}\right\|_{E}+\left\|\sum_{k=1}^{n} f_{k}^{2}(t) e_{k}\right\|_{E}+\left\|\sum_{k=1}^{n} f_{k}^{3}(t) e_{k}\right\|_{E}\right),
$$

то утверждение теоремы 30 следует из соотношений (73) и (75).

СлеДСтвиЕ 7. Пусть функииональное симметричное пространство $X$ имеет свойство Круглова, а $E$ - произвольное (банахово) симметричное пространство последовательностей. Тогда существует константа $C>0$, зависящая только от $X$, такая, что для всех $n \in \mathbb{N}$ и произвольной последовательности независимых функиий $\left\{f_{k}\right\}_{k=1}^{n} \subset X$ верно следующее неравенство:

$$
\begin{gathered}
C^{-1}\left(\left\|\mathbf{f}^{*} \chi_{[0,1]}\right\|_{X}+\|\| \sum_{k=1}^{n} f_{k} e_{k}\left\|_{E}\right\|_{L_{1}}\right) \leqslant\|\| \sum_{k=1}^{n} f_{k} e_{k}\left\|_{E}\right\|_{X} \\
\leqslant C\left(\left\|\mathbf{f}^{*} \chi_{[0,1]}\right\|_{X}+\|\| \sum_{k=1}^{n} f_{k} e_{k}\left\|_{E}\right\|_{L_{1}}\right) .
\end{gathered}
$$

ДоказАтельство. Левое неравенство в (76) верно для любого симметричного пространства $X$ и является непосредственным следствием предложения 7 , а также того, что $\|f\|_{L_{1}} \leqslant\|f\|_{X}(f \in X)$. Чтобы получить правую оценку, заметим, что ввиду соотношения (61), примененного в частном случае $X=L_{1}$,

$$
\left\|\sum_{k=1}^{n} \mathbf{f}^{*}(k) e_{k}\right\|_{E} \leqslant C^{\prime}\|\| \sum_{k=1}^{n} f_{k} e_{k}\left\|_{E}\right\|_{L_{1}} .
$$

Нужный нам результат следует отсюда и из неравенства (67).

ЗАмечАниЕ 8. Оценка, полученная в теореме 30 (и точно так же оценка сверху в следствии 7), точна в следующем смысле. Если она верна в случае $E=\ell_{1}$ для каждого $n \in \mathbb{N}$ и произвольной последовательности независимых функций $\left\{f_{k}\right\}_{k=1}^{n} \subset X$, где $X$ - сепарабельное или максимальное симметричное пространство на $[0,1]$, то $X$ имеет свойство Круглова (см. теоремы 22 и 23).

ЗАмечАниЕ 9. Если банаховы пространства $X$ и $E$ максимальны, то, как нетрудно показать, неравенства (61), (67) и (76) будут верны также для бесконечных последовательностей $\left\{f_{k}\right\}_{k=1}^{\infty} \subset X$. 


\section{7. Неравенство Хинчина и его обобщения}

Пусть, как и ранее, $r_{k}(t)=\operatorname{sign} \sin 2^{k} \pi t(k=1,2, \ldots)$ - функции Радемахера, определенные на отрезке $[0,1]$. Неравенства Хинчина (1), приведенные в разделе 1, вызвали огромное количество исследований и обобщений, нашли многочисленные применения в самых различных разделах анализа (см., например, [3]). В частности, в 1975 г. В. А. Родин и Е. М. Семенов [66] доказали, что для симметричного пространства $X$ на $[0,1]$ оценка сверху

$$
\left\|\sum_{k=1}^{\infty} a_{k} r_{k}\right\|_{X} \leqslant C\left(\sum_{k=1}^{\infty} a_{k}^{2}\right)^{1 / 2}
$$

имеет место тогда и только тогда, когда $X$ содержит пространство $\left(\exp L_{2}\right)$ 。

Доказанная в разделе 5 теорема 25 позволяет найти точные условия на симметричное пространство $X$, при которых в нем справедливо аналогичное неравенство с заменой скалярных кратных функций Радемахера произвольными независимыми функциями, в среднем равными нулю. Фактически мы рассмотрим вопрос о справедливости даже более общей оценки, когда норма $\ell_{2}$ в правой части (77) заменена на норму произвольного симметричного пространства последовательностей $E \neq \ell_{1}$. Следующая теорема была доказана в работе [67] (см. также [68]).

Теорема 31. Пусть $X$ - произволъное сепарабелъное или максимальное симметричное пространство на $[0,1]$. Следующие условия эквивалентны:

(a) $X \in \mathbb{K}$;

(b) существует такое $C>0$, что для любой последовательности независимых функиици $\left\{f_{k}\right\}_{k=1}^{\infty} \subset X, \int_{0}^{1} f_{k}(t) d t=0(k=1,2, \ldots)$, выполнено:

$$
\left\|\sum_{k=1}^{\infty} f_{k}\right\|_{X} \leqslant C\left\|\left(\sum_{k=1}^{\infty} f_{k}^{2}\right)^{1 / 2}\right\|_{X}
$$

(c) существуют такие симметричное пространство $E \neq \ell_{1}$ и константа $C>0$, что для любой последовательности независимых функиий $\left\{f_{k}\right\}_{k=1}^{\infty} \subset X$, $\int_{0}^{1} f_{k}(t) d t=0 \quad(k=1,2, \ldots)$, выполнено:

$$
\left\|\sum_{k=1}^{\infty} f_{k}\right\|_{X} \leqslant C\|\|\left(f_{k}\right)\left\|_{E}\right\|_{X}
$$

(d) существуют такие симметричное пространство $E \neq \ell_{1}$ и константа $C>0$, что (79) выполнено для любой последовательности симметрично распределенных независимых функиий $\left\{f_{k}\right\}_{k=1}^{\infty} \subset X$, удовлетворяющей условию

$$
\sum_{k=1}^{\infty} \lambda\left(\operatorname{supp} f_{k}\right) \leqslant 1 \text {. }
$$


Далее нам понадобятся две леммы, первую из которых мы здесь только сформулируем (доказательство см. в [67]).

Лемма 16. Пусть $X$ - симметричное пространство на $[0,1]$, а $E$ - симметричное пространство последовательностей, $E \neq \ell_{1}$. Предположим, что для некоторого $C>0$ и для любой последовательности симметрично распределенных независимых функиий $\left\{f_{k}\right\}_{k=1}^{\infty} \subset X$, удовлетворяющей условию $(80)$, выполнено (79). Тогда аналогичное неравенство (возможно, с иной константой) имеет место и для произвольной последовательности независимых функиий, удовлетворяющей условию (80).

Второе утверждение можно получить, используя общие соображения, связанные с теорией интерполяции операторов (см., например, [69; п. 12]), но мы предпочитаем дать его прямое доказательство.

Лемма 17. Пусть $E$ - симметричное пространство последовательностей, $E \neq \ell_{1}$. Тогда существует положительная однородная функиия первой степени $\psi(u, v)$, возрастающая по каждому аргументу, такая, что $\lim _{u \rightarrow 0+} \psi(u, v)=0$ и для всех $a=\left(a_{k}\right)_{k=1}^{\infty} \in \ell_{1}$

$$
\|a\|_{E} \leqslant \psi\left(\|a\|_{\ell_{\infty}},\|a\|_{\ell_{1}}\right) .
$$

ДокАЗАтельство. Пусть $\phi_{E}-$ фундаментальная функция пространства $E$, т. е. $\phi_{E}(n)=\left\|\sum_{k=1}^{n} e_{k}\right\|_{E}(n \in \mathbb{N})$, где $e_{k}-$ стандартные орты в пространстве последовательностей. Рассмотрим кусочно линейную функцию $\varphi(t)$ на $[0, \infty)$ такую, что $\varphi(0)=0$ и $\varphi(n)=\phi(n)(n \in \mathbb{N})$. Так как $\phi(n)$ возрастает, а $\phi(n) / n$ убывает, то $\varphi(t)$ квазивогнута при $t \geqslant 0$. Поэтому, если $\bar{\varphi}(t)-$ ее наименьшая вогнутая мажоранта, то (см. раздел 2 или [16; теорема 2.1.1])

$$
\varphi(t) \leqslant \bar{\varphi}(t) \leqslant 2 \varphi(t) \quad(t>0) .
$$

Легко проверить, что $\psi(u, v):=v \bar{\varphi}(u / v)(u, v>0)$ - положительная однородная функция первой степени, возрастающая по каждому аргументу. Кроме того, ввиду (82) и определения $\varphi$ имеем: $\lim _{u \rightarrow 0+} \psi(u, v)=0$.

Докажем соотношение (81). Пусть $a=\left(a_{k}\right)_{k=1}^{\infty} \in \ell_{1}$. Не ограничивая общности, можно считать, что $a_{1} \geqslant a_{2} \geqslant \cdots \geqslant 0$. Для каждого $n \in \mathbb{N}$ определим $a^{n}=\sum_{k=1}^{n} a_{k} e_{k}$. Так как $a^{n}=\sum_{k=1}^{n}\left(a_{k}-a_{k+1}\right) \sum_{i=1}^{k} e_{i}$, где $a_{n+1}=0$, то ввиду вогнутости $\bar{\varphi}(t)$, соотношения $(82)$, а также определения и свойств функции $\psi$ получаем:

$$
\begin{aligned}
\left\|a^{n}\right\|_{E} & \leqslant \sum_{k=1}^{n}\left(a_{k}-a_{k+1}\right) \varphi(k) \leqslant a_{1} \sum_{k=1}^{n} \frac{a_{k}-a_{k+1}}{a_{1}} \bar{\varphi}(k) \\
& \leqslant a_{1} \bar{\varphi}\left(\sum_{k=1}^{n} \frac{a_{k}-a_{k+1}}{a_{1}} k\right)=\psi\left(a_{1}, \sum_{k=1}^{n}\left(a_{k}-a_{k+1}\right) k\right) \leqslant \psi\left(\|a\|_{\ell_{\infty}},\|a\|_{\ell_{1}}\right) .
\end{aligned}
$$

Из того, что $\left\|a^{n}-a\right\|_{\ell_{1}} \rightarrow 0$, следует: $\left\|a^{n}-a\right\|_{E} \rightarrow 0$, и поэтому, переходя в последнем неравенстве к пределу при $n \rightarrow \infty$, получаем (81). Лемма доказана. 
ДОКАЗАТЕЛЬСТво теОРЕмЫ 31. (a) $\Rightarrow$ (b). Пусть $\left\{f_{k}\right\}_{k=1}^{\infty} \subset X$ состоит из таких независимых функций, что $\int_{0}^{1} f_{k}(t) d t=0 \quad(k=1,2, \ldots)$. Тогда по теореме 25 существует $C>0$, зависящее лишь от пространства $X$, такое, что

$$
\left\|\sum_{k=1}^{\infty} f_{k}\right\|_{X} \leqslant C\left\|\sum_{k=1}^{\infty} \mathbf{f}_{k}\right\|_{Z_{X}^{2}}
$$

Напомним, что $\mathbf{f}_{k}(t)=f_{k}(t-k+1) \chi_{[k-1, k)}(t)(t>0)$, а $Z_{X}^{2}$ - симметричное пространство на $(0, \infty)$ с квазинормой $\|g\|_{Z_{X}^{2}}:=\left\|g^{*} \chi_{[0,1]}\right\|_{X}+\left\|g^{*} \chi_{[1, \infty)}\right\|_{L_{2}[1, \infty)}$. Поэтому, если $\mathbf{f}=\sum_{k=1}^{\infty} \mathbf{f}_{k}$, то по теореме 26 с $E=\ell_{2}$ получаем:

$$
\left\|\sum_{k=1}^{\infty} \mathbf{f}_{k}\right\|_{Z_{X}^{2}} \leqslant\left\|\mathbf{f}^{*} \chi_{[0,1]}\right\|_{X}+\left(\sum_{k=1}^{\infty} \mathbf{f}^{*}(k)^{2}\right)^{1 / 2} \leqslant 6\left\|\left(\sum_{k=1}^{\infty} f_{k}^{2}\right)^{1 / 2}\right\|_{X} .
$$

Отсюда и из (83) сразу следует (78), и (b) доказано.

Импликации (b) $\Rightarrow$ (c) и (c) $\Rightarrow$ (d) очевидны.

$(\mathrm{d}) \Rightarrow(\mathrm{a})$. По условию и лемме 16 соотношение (31) выполнено для произвольной последовательности независимых функций, удовлетворяющей условию (80). Применяя (31) к функциям $\left|f_{k}\right|(k=1,2, \ldots)$ и учитывая $(81)$, находим, что

$$
\left\|\sum_{k=1}^{\infty}\left|f_{k}\right|\right\|_{X} \leqslant C\|\|\left(f_{k}\right)\left\|_{E}\right\|_{X} \leqslant C\left\|\psi\left(\left\|\left(f_{k}\right)\right\|_{l_{\infty}},\left\|\left(f_{k}\right)\right\|_{l_{1}}\right)\right\|_{X} .
$$

Пусть $x, y \in X, x \neq 0, y \neq 0$. Так как ввиду свойств функции $\psi$ для произвольных $s, t>0$

$$
\psi(x, y) \leqslant \max \left\{\frac{x}{s}, \frac{y}{t}\right\} \psi(s, t) \leqslant\left(\frac{x}{s}+\frac{y}{t}\right) \psi(s, t),
$$

то, взяв $s$ и $t$ так, что $\|x\| / s=\|y\| / t$, получим

$$
\|\psi(x, y)\| \leqslant\left(\frac{\|x\|}{s}+\frac{\|y\|}{t}\right) \psi(s, t) \leqslant 2 \max \left\{\frac{\|x\|}{s}, \frac{\|y\|}{t}\right\} \psi(s, t)=2 \psi(\|x\|,\|y\|) .
$$

Тем самым из (84) следует:

$$
\begin{aligned}
\left\|\sum_{k=1}^{\infty}\left|f_{k}\right|\right\|_{X} & \leqslant 2 C \psi\left(\|\|\left(f_{k}\right)\left\|_{\ell_{\infty}}\right\|_{X},\|\|\left(f_{k}\right)\left\|_{\ell_{1}}\right\|_{X}\right) \\
& =2 C\left\|\sum_{k=1}^{\infty}\left|f_{k}\right|\right\|_{X} \psi\left(\frac{\|\|\left(f_{k}\right)\left\|_{\ell_{\infty}}\right\|_{X}}{\|\|\left(f_{k}\right)\left\|_{\ell_{1}}\right\|_{X}}, 1\right),
\end{aligned}
$$

откуда

$$
\psi\left(\frac{\|\|\left(f_{k}\right)\left\|_{\ell_{\infty}}\right\|_{X}}{\|\|\left(f_{k}\right)\left\|_{\ell_{1}}\right\|_{X}}, 1\right) \geqslant \frac{1}{2 C}
$$


Так как по лемме $17 \lim _{u \rightarrow 0+} \psi(u, v)=0$, то из последнего неравенства следует, что для некоторого $C_{1}>0$

$$
\left\|\sum_{k=1}^{\infty}\left|f_{k}\right|\right\|_{X} \leqslant C_{1}\left\|\sup _{k=1,2, \ldots}\left|f_{k}\right|\right\|_{X} .
$$

Поэтому ввиду предложения 7 неравенство

$$
\left\|\sum_{k=1}^{\infty}\left|f_{k}\right|\right\|_{X} \leqslant C_{1}\left\|\sum_{k=1}^{\infty} \mathbf{f}_{k}\right\|_{X}
$$

выполнено для произвольной последовательности независимых функций, удовлетворяющих условию (4). Так как пространство $X$ максимально или сепарабельно, то, применяя теоремы 22 и 23 , получаем, что $X \in \mathbb{K}$. Теорема доказана.

ЗАмечАниЕ 10 . Если в качестве $f_{k}(k=1,2, \ldots)$ брать лишь скалярные кратные функций Радемахера, то результат будет совсем иным. Как доказано в [70], для каждого пространства последовательностей $E$, интерполяционного относительно пары $\left(\ell_{1}, \ell_{2}\right)$, найдется функциональное симметричное пространство $X$ на $[0,1]$, для которого имеет место двусторонняя оценка $C^{-1}\left\|\left(a_{k}\right)\right\|_{E} \leqslant$ $\left\|\sum_{k=1}^{\infty} a_{k} r_{k}\right\|_{X} \leqslant C\left\|\left(a_{k}\right)\right\|_{E}$. Поэтому, разумеется, оценка справа в последнем неравенстве для некоторого $E \neq \ell_{1}$ ни в коей мере не гарантирует того, что аналогичная оценка будет верна и для $\ell_{2}$.

Применим теперь теорему 31 к изучению векторнозначных рядов вида

$$
F(s, t)=\sum_{k=1}^{\infty} f_{k}(s) r_{k}(t) \quad(s, t \in[0,1])
$$

где $\left\{f_{k}\right\}$ - произвольная последовательность независимых функций, а $r_{k}-$ функции Радемахера. Если $X$ - симметричное пространство на $I=[0,1]$, то $X(I \times I)$ - соответствующее симметричное пространство на квадрате $I \times I$ с нормой $\|f\|_{X(I \times I)}=\left\|f^{*}\right\|_{X}$.

Теорема 32. Если симметричное пространство $X$ сепарабельно или максимально, то следующие условия эквивалентны:

( $\alpha) X \in \mathbb{K}$

( $\beta$ ) существует такое $C>0$, что для любой последовательности независимых функиий $\left\{f_{k}\right\}_{k=1}^{\infty} \subset X$ выполнено:

$$
C^{-1}\left\|\left(\sum_{k=1}^{\infty} f_{k}^{2}\right)^{1 / 2}\right\|_{X} \leqslant\left\|\sum_{k=1}^{\infty} f_{k}(s) r_{k}(t)\right\|_{X(I \times I)} \leqslant C\left\|\left(\sum_{k=1}^{\infty} f_{k}^{2}\right)^{1 / 2}\right\|_{X} ;
$$

$(\gamma)$ существует такое $C>0$, что для любой последовательности независимых функиий $\left\{f_{k}\right\}_{k=1}^{\infty} \subset X$ выполнено:

$$
C^{-1}\left\|\left(\sum_{k=1}^{\infty} f_{k}^{2}\right)^{1 / 2}\right\|_{X} \leqslant \int_{0}^{1}\left\|\sum_{k=1}^{\infty} f_{k}(s) r_{k}(t)\right\|_{X} d t \leqslant C\left\|\left(\sum_{k=1}^{\infty} f_{k}^{2}\right)^{1 / 2}\right\|_{X} .
$$


ДокАзАтЕльство. Прежде всего, заметим, что левые неравенства в (85) и (86) имеют место в любом симметричном пространстве. Проверим это, например, для (85). Ввиду безусловности последовательности $\left\{f_{k}(s) r_{k}(t)\right\}_{k=1}^{\infty}$ в $X(I \times I)$ с константой 1 [39; предложение 1.14], а также неравенств Хинчина (1) для $L_{1}$ с оптимальной константой $A_{1}=1 / \sqrt{2}$ из [71] имеем:

$$
\begin{array}{r}
\left\|\sum_{k=1}^{\infty} f_{k}(s) r_{k}(t)\right\|_{X(I \times I)}=\int_{0}^{1}\left\|\sum_{k=1}^{\infty} f_{k}(s) r_{k}(t) r_{k}(u)\right\|_{X(I \times I)} d u \\
\geqslant\left\|\int_{0}^{1}\left|\sum_{k=1}^{\infty} f_{k}(s) r_{k}(t) r_{k}(u)\right| d u\right\|_{X(I \times I)} \geqslant \frac{1}{\sqrt{2}}\left\|\left(\sum_{k=1}^{\infty} f_{k}^{2}\right)^{1 / 2}\right\|_{X} .
\end{array}
$$

Доказательство левой части (86) совершенно аналогично.

Далее, так как функции $f_{k}(s) r_{k}(t)(k=1,2, \ldots)$ независимы и

$$
\int_{0}^{1} \int_{0}^{1} f_{k}(s) r_{k}(t) d s d t=0 \quad(k=1,2, \ldots),
$$

то по теореме 31 правое неравенство в (85) - непосредственное следствие $(\alpha)$. Докажем правое неравенство в (86). Пусть $\left\{f_{k}\right\}_{k=1}^{\infty} \subset X-$ последовательность независимых функций. Тогда функции $g_{k}(s)=f_{k}(s)-\int_{0}^{1} f_{k}(t) d t(k=1,2, \ldots)$ также независимы и, кроме того, $\int_{0}^{1} g_{k}(t) d t=0, k=1,2, \ldots$. Поэтому, применяя теорему 31 к последовательности $\left\{g_{k}(\cdot) r_{k}(t)\right\}_{k=1}^{\infty}$ для фиксированного $t \in[0,1]$ и учитывая в очередной раз, что $\left\|\chi_{[0,1]}\right\|_{X}=1$ и $\|g\|_{X} \geqslant \int_{0}^{1}|g(s)| d s$,

$$
\begin{aligned}
& \int_{0}^{1} \| \sum_{k=1}^{\infty} f_{k}(s) r_{k}(t)\left\|_{X} d t=\int_{0}^{1}\right\| \sum_{k=1}^{\infty}\left(g_{k}(s)+\int_{0}^{1} f_{k}(u) d u\right) r_{k}(t) \|_{X} d t \\
& \leqslant \int_{0}^{1}\left\|\sum_{k=1}^{\infty} g_{k}(s) r_{k}(t)\right\|\left\|_{X} d t+\int_{0}^{1} \mid \sum_{k=1}^{\infty} \int_{0}^{1} f_{k}(u) d u \cdot r_{k}(t)\right\|_{d} \\
& \leqslant C\left\|\left(\sum_{k=1}^{\infty} g_{k}^{2}\right)^{1 / 2}\right\|_{X}+\left(\sum_{k=1}^{\infty}\left(\int_{0}^{1} f_{k}(u) d u\right)^{2}\right)^{1 / 2} \leqslant C\left\|\left(\sum_{k=1}^{\infty} f_{k}^{2}\right)^{1 / 2}\right\|_{X} \\
&+(C+1)\left(\sum_{k=1}^{\infty}\left(\int_{0}^{1} f_{k}(u) d u\right)^{2}\right)^{1 / 2} \leqslant(2 C+1)\left\|\left(\sum_{k=1}^{\infty} f_{k}^{2}\right)^{1 / 2}\right\|_{X}
\end{aligned}
$$

Пусть, наоборот, выполнено $(\beta)$ или $(\gamma)$ (точнее, правая часть (85) или $(86)$ ). Для доказательства $(\alpha)$ достаточно проверить, что пространство $X$ удовлетворяет условию (d) теоремы 31. Предположим, что последовательность $\left\{f_{k}\right\}_{k=1}^{\infty} \subset X$ состоит из симметрично распределенных независимых функций, удовлетворяющих (80). Еще раз применяя [39; предложение 1.14], заключаем, что $\left\{f_{k}\right\}$ безусловна с константой 1 в $X$. Поэтому, если выполнена правая часть (86), то

$$
\left\|\sum_{k=1}^{\infty} f_{k}\right\|_{X}=\int_{0}^{1}\left\|\sum_{k=1}^{\infty} f_{k}(s) r_{k}(t)\right\|_{X} d t \leqslant C\left\|\left(\sum_{k=1}^{\infty} f_{k}^{2}\right)^{1 / 2}\right\|_{X}
$$


и $(\mathrm{d})$ доказано. Кроме того, распределение $f_{k}(s)$ на $I$ совпадает с распределением $f_{k}(s) r_{k}(t)$ на $I \times I(k=1,2, \ldots)$. Действительно, так как $f_{k}$ симметрично распределены, то для любого $\tau \in \mathbb{R}$

$$
\begin{aligned}
& \lambda^{2}\left\{(s, t) \in I \times I: f_{k}(s) r_{k}(t)>\tau\right\} \\
& \quad=\frac{1}{2}\left(\lambda\left\{s \in I: f_{k}(s)>\tau\right\}+\lambda\left\{s \in I:-f_{k}(s)>\tau\right\}\right)=\lambda\left\{s \in I: f_{k}(s)>\tau\right\} .
\end{aligned}
$$

Значит, ввиду независимости функций $f_{k}(k=1,2, \ldots)$ и $f_{k}(s) r_{k}(t)(k=$ $1,2, \ldots$ ) соответственно получаем

$$
\left\|\sum_{k=1}^{\infty} f_{k}\right\|_{X}=\left\|\sum_{k=1}^{\infty} f_{k}(s) r_{k}(t)\right\|_{X(I \times I)},
$$

и тем самым (d) - следствие также и правой части (85). Теорема доказана.

ЗАмечАниЕ 11. Утверждения последней теоремы интересно сопоставить с двумя хорошо известными результатами. Ввиду первого из них в любом сепарабельном или максимальном симметричном пространстве $X$ следующие условия эквивалентны: (i) существует такое $C>0$, что для произвольной последовательности функций $\left\{f_{k}\right\}_{k=1}^{\infty} \subset X$ выполнено соотношение (85), и (ii) нижний индекс Бойда $\alpha_{X}$ положителен (импликация (ii) $\Rightarrow$ (i) доказана в [17; предложение 2.d.1], а импликация (i) $\Rightarrow$ (ii) - в [72]). Второй (неравенство Морэ) говорит о том, что если $X$ является $q$-вогнутой банаховой решеткой $[17$; определение 1.d.3] при некотором $q<\infty$, то существует такое $C>0$, что (86) выполнено для произвольной последовательности функций $\left\{f_{k}\right\}_{k=1}^{\infty} \subset X[17$; теорема 1.d.6(i)]. Заметим, что, с одной стороны, в обоих упомянутых утверждениях коэффициенты ряда - произволъные функции, а с другой, условия выполнения неравенств (85) и (86) в них гораздо более ограничительные, чем условие $(\alpha)$ теоремы 32 .

В симметричных пространствах, “близких" к $L_{\infty}$, неравенство Морэ, о котором шла речь в предыдущем абзаце, вообще говоря, неверно. Тем не менее, в последние годы были получены оценки норм полиномов по системам независимых функций и в таких пространствах. Об этом речь пойдет в следующем разделе.

\section{8. Оценки норм полиномов по системам независимых функций в пространствах, “близких" к $L_{\infty}$}

Пусть $\left\{\xi_{i}\right\}_{i=1}^{n}-$ система независимых случайных величин на вероятностном пространстве $(T, \mathscr{T}, \tau)$, а $\left\{f_{i}\right\}_{i=1}^{n}$ - система измеримых функций на другом вероятностном пространстве $(X, \Sigma, \mu)$. Рассмотрим математическое ожидание (относительно $\tau$ )

$$
\mathrm{E}\left\|\sum_{i=1}^{n} \xi_{i}(t) f_{i}(x)\right\|_{L_{\infty}(\mu)} .
$$


Классический результат Р. Салема и А. Зигмунда [73] утверждает, что для некоторого $C>0$ и всех $n \in \mathbb{N}$

$$
C^{-1}(n \ln n)^{1 / 2} \leqslant \mathrm{E}\left\|\sum_{j=1}^{n} r_{j}(t) e^{2 \pi i j x}\right\|_{L_{\infty}} \leqslant C(n \ln n)^{1 / 2},
$$

где, как и ранее, $r_{i}(t)$ - функции Радемахера на $[0,1]$.

В монографиях [74] и [75] детально изучена более общая ситуация, связанная с поведением величины (87) в случае, когда $\left\{f_{i}\right\}$ - система характеров на локально компактной абелевой группе $G$, определенных в компактной симметричной окрестности $V$ единичного элемента $e \in G$. Доказательство нижних оценок для величины (87) при этом основывается на ее сравнении с величиной

$$
\mathrm{E}\left\|\sum_{i=1}^{n} \gamma_{i}(t) f_{i}(x)\right\|_{L_{\infty}(\mu)},
$$

где $\left\{\gamma_{i}\right\}_{i=1}^{\infty}$ - система независимых гауссовских с.в., $\mathrm{E} \gamma_{i}=0, \mathrm{E} \gamma_{i}^{2}=1(i=$ $1,2, \ldots)$. Решающее значение при этом имеют различные варианты известной леммы Слепяна, позволяющие оценивать снизу выражения вида

$$
\mathrm{E} \max _{1 \leqslant k \leqslant m}\left|\sum_{i=1}^{n} \gamma_{i}(t) f_{i}\left(x_{k}\right)\right|
$$

а тем самым и величину (89) при условии, что точки $x_{1}, \ldots, x_{m}$ выбраны в $V$ так, что векторы

$$
W_{x_{k}}=\left(f_{1}\left(x_{k}\right), \ldots, f_{n}\left(x_{k}\right)\right) \in \mathbb{C}^{n} \quad(k=1, \ldots, m)
$$

расположены достаточно далеко друг от друга в евклидовой метрике $\mathbb{C}^{n}$. Таким образом, оценка снизу для (89) может быть получена только при условии контроля $\varepsilon$-энтропии в $\ell_{2}^{n}$-метрике множества $\left\{W_{x}\right\}_{x \in V}$. Последнее является нетривиальной задачей даже в относительно простых случаях.

В работах Б. С. Кашина и Л. Цафрири [76] и [77] для решения рассматриваемой задачи был предложен новый интересный метод, основанный на применении версии двумерной центральной предельной теоремы с оценкой остаточного члена. Оказалось, что при его использовании достаточно обеспечить малость среднего

$$
\frac{1}{n^{2}} \sum_{i, j=1}^{n}\left|\left(W_{x_{i}}, W_{x_{j}}\right)\right|^{2}
$$

Последнее сделать, конечно, гораздо легче, чем гарантировать выполнение аналогичного условия для энтропии множества $\left\{W_{x_{i}}\right\}_{i=1}^{n}$, т. е. малость всех скалярных произведений $\left(W_{x_{i}}, W_{x_{j}}\right) \quad(1 \leqslant i \neq j \leqslant n)$. Благодаря этому удается показать, что нижняя оценка в (88) остается справедливой для случайных полиномов по любой ортонормированной ограниченной в $L_{3}$ системе. Приведем формулировку теоремы, доказанной в [76]. 
Теорема 33. Для любого $M>0$ существуют константы $C_{j}=C_{j}(M)>0$ $(j=1,2,3)$ и $q=q(M)>0$ со следующим свойством: для каждой системь функций $\left\{f_{i}\right\}_{i=1}^{n}$ такой, что

(a) $\left\|f_{i}\right\|_{L_{2}(\mu)}=1 u\left\|f_{i}\right\|_{L_{3}(\mu)} \leqslant M(i=1, \ldots, n)$,

(b) $\left\|\sum_{i=1}^{n} a_{i} f_{i}\right\|_{L_{2}(\mu)} \leqslant M\left(\sum_{i=1}^{n}\left|a_{i}\right|^{2}\right)^{1 / 2}$ для всех $a_{i} \in \mathbb{C}$,

и каждой последовательности с.в. $\left\{\xi_{i}\right\}_{i=1}^{n}$, определенных на вероятностном пространстве $(T, \mathscr{T}, \tau)$, такой, что

(с) $\xi_{i}$ независимы, $\mathrm{E} \xi_{i}=0, \mathrm{E}\left|\xi_{i}\right|^{2}=1 u\left(\mathrm{E}\left|\xi_{i}\right|^{3}\right)^{1 / 3} \leqslant M(i=1, \ldots, n)$, выполнено:

$$
\tau\left\{t \in T:\left\|\sum_{i=1}^{n} \xi_{i}(t) f_{i}\right\|_{L_{\infty}(\mu)} \leqslant C_{1}(n \ln n)^{1 / 2}\right\} \leqslant C_{2} n^{-q},
$$

и, в частности,

$$
\mathrm{E}\left\|\sum_{i=1}^{n} \xi_{i} f_{i}\right\|_{L_{\infty}(\mu)} \geqslant C_{3}(n \ln n)^{1 / 2} .
$$

В работе [77] было получено обобщение последней теоремы для "случайных" норм вида

$$
\left\|\sum_{i=1}^{n} \xi_{i}(t) a_{i} f_{i}(x)\right\|_{L_{\infty}(\mu)},
$$

где $a_{i} \in \mathbb{C}$. Там же было отмечено, что на самом деле аналогичные оценки снизу верны не только для $L_{\infty}$-нормы, но и для введенной там же так называемой интегрально-равномерной нормы

$$
\|f\|_{m, \infty}=\int_{X} \cdots \int_{X} \max \left\{\left|f\left(x_{1}\right)\right|, \ldots,\left|f\left(x_{m}\right)\right|\right\} d \mu\left(x_{1}\right) \cdots d \mu\left(x_{m}\right),
$$

где $m \in \mathbb{N}$, a $f$ - произвольная интегрируемая функция на вероятностном пространстве $(X, \Sigma, \mu)$. Несомненно, что оценки для норм $\|\cdot\|_{m, \infty}$ представляют значительный интерес, так как последние несут в себе содержательную информацию о распределении функции $f$. Прежде всего, как нетрудно показать,

$$
\|f\|_{m, \infty}=\int_{0}^{\infty}\left(1-\left(1-n_{f}(z)\right)^{m}\right) d z
$$

где $n_{f}(z)=\mu\{x \in X:|f(x)|>z\}$. Отсюда, в частности, следует, что $\|f\|_{1} \leqslant$ $\|f\|_{m, \infty} \leqslant\|f\|_{\infty}$ и $\|f\|_{m, \infty} \rightarrow\|f\|_{\infty}$ при $m \rightarrow \infty$. Кроме того, как показано в [78; предложение 2] и независимо в [79; теорема 2], с константами, не зависящими от $m \in \mathbb{N}$,

$$
\|f\|_{m, \infty} \asymp m \int_{0}^{1 / m} f^{*}(t) d t .
$$

Следующее обобщение результатов Б. С. Кашина и Л. Цафрири было получено П. Г. Григорьевым в [80; теорема 1] (см. также [81; теорема 1]). 
ТЕОРема 34. Пусть $\left\{f_{i}\right\}_{i=1}^{n} u\left\{\xi_{i}\right\}_{i=1}^{n}-$ множества функиий, определенных на вероятностных пространствах $(X, \Sigma, \mu)$ u $(T, \mathscr{T}, \tau)$ соответственно и удовлетворяющих условиям (а) $u$ (с) теоремы 33. Предположим также, что набор $\left(a_{i}\right)_{i=1}^{n} \in \mathbb{C}^{n}$ зафиксирован и для всех $\left(c_{i}\right)_{i=1}^{n} \in \mathbb{C}^{n}$ выполнено неравенство

$$
\left\|\sum_{i=1}^{n} c_{i} f_{i}\right\|_{L_{2}(\mu)} \leqslant \operatorname{MR}\left(\left\{a_{i}\right\}\right)^{p}\left(\sum_{i=1}^{n}\left|c_{i}\right|^{2}\right)^{1 / 2},
$$

где $M>0$ и $p \in[0,1 / 2)$ - некоторые константь, а

$$
R=R\left(\left\{a_{i}\right\}\right)=\left(\sum_{i=1}^{n}\left|a_{i}\right|^{2}\right)^{2}\left(\sum_{i=1}^{n}\left|a_{i}\right|^{4}\right)^{-1} .
$$

Тогда существуют константы $q=q(p, M)>0, C_{j}=C_{j}(p, M)>0 \quad(j=$ 1,2,3), для которых справедливы следующие неравенства:

$$
\tau\left\{t \in T:\left\|\sum_{i=1}^{n} a_{i} \xi_{i}(t) f_{i}\right\|_{m, \infty} \leqslant C_{1}\left(\sum_{i=1}^{n}\left|a_{i}\right|^{2} \ln P\right)^{1 / 2}\right\} \leqslant \frac{C_{2}}{P^{q}}
$$

$u$

$$
\mathrm{E}\left\|\sum_{i=1}^{n} a_{i} \xi_{i} f_{i}\right\|_{m, \infty} \geqslant C_{3}\left(\sum_{i=1}^{n}\left|a_{i}\right|^{2} \ln P\right)^{1 / 2},
$$

где $P:=\min \{m, R\}+1$.

В той же работе показано, что при $m \leqslant n$ и некоторых ограничениях на последовательность $\left\{\xi_{i}\right\}_{i=1}^{n}$ оценка (92) точна по порядку.

ТеОрема 35. Пусть $\xi_{i}(i=1, \ldots, n)$ - независимые с.в., для распределений полиномов относительно которых справедлива экспоненциальная оценка:

$$
\tau\left\{t \in T:\left|\sum_{i=1}^{n} c_{i} \xi_{i}(t)\right| \geqslant s\left(\sum_{i=1}^{n}\left|c_{i}\right|^{2}\right)^{1 / 2}\right\} \leqslant C_{4} e^{-C_{5} s^{2}} \quad(s>0),
$$

где константы $C_{4}>0$ и $C_{5}>0$ не зависят от последовательности коэфбициентов $\left\{c_{i}\right\}_{i=1}^{n} \in \mathbb{C}^{n}$. Тогда существует такая абсолютная константа $C_{6}>0$, что для каждого $m \geqslant 1$ и произвольных $f_{i} \in L_{1}(X)(i=1, \ldots, n)$ выполнено:

$$
\mathrm{E}\left\|\sum_{i=1}^{n} \xi_{i} f_{i}\right\|_{m, \infty} \leqslant C_{6}\left\|\left(\sum_{i=1}^{n}\left|f_{i}\right|^{2}\right)^{1 / 2}\right\|_{m, \infty} \sqrt{1+\ln m} .
$$

В частности, если $f_{i} \in L_{\infty}(X)(i=1, \ldots, n)$, mо

$$
\mathrm{E}\left\|\sum_{i=1}^{n} \xi_{i} f_{i}\right\|_{m, \infty} \leqslant C_{6}\left(\sum_{i=1}^{n}\left\|f_{i}\right\|_{\infty}^{2}\right)^{1 / 2} \sqrt{1+\ln m} .
$$

СЛЕДСТВИЕ 8. Предположим, что $\left\|f_{i}\right\|_{L_{\infty}(X)} \leqslant M(i=1, \ldots, n)$, a с.в. $\xi_{i}$ $(i=1, \ldots, n)$ независимы и удовлетворяют экспоненциальной оценке (93). Тогда для произвольных $a_{i} \in \mathbb{C}$

$$
\mathrm{E}\left\|\sum_{i=1}^{n} a_{i} \xi_{i} f_{i}\right\|_{m, \infty} \leqslant M C_{6}\left(\sum_{i=1}^{n}\left|a_{i}\right|^{2}\right)^{1 / 2} \sqrt{1+\ln m} .
$$


Тем самым, если $m \leqslant n$ u $m \leqslant C_{7} R\left(\left\{a_{i}\right\}_{i=1}^{n}\right)$ (см. теорему 34), то неравенство (92) из теоремы 34 при этих условиях точно по порядку.

В работе [79] в случае $a_{i}=1(i=1, \ldots, n)$ П. Г. Григорьеву удалось распространить утверждение теоремы 34 на системы $\left\{f_{i}\right\}$, вообще говоря, неограниченные в $L_{p}$ при $p>1$.

ТЕОРема 36. Пусть последовательность функиий $\left\{f_{i}\right\}_{i=1}^{n}$, определенных на вероятностном пространстве $(X, \Sigma, \mu)$, такова, что $\left\|f_{i}\right\|_{L_{1}(\mu)}=1 \quad(i=$ $1, \ldots, n) u\left\|\sum_{i=1}^{n} \theta_{i} f_{i}\right\|_{L_{1}(\mu)} \leqslant M n^{1 / 2+p}$ для всех наборов знаков $\left(\theta_{i}\right)_{i=1}^{n}, \theta_{i}= \pm 1$, с некоторыми константами $M>0$ и $p \in[0,1 / 12)$. Кроме того, предположим, что последовательность $\left\{\xi_{i}\right\}_{i=1}^{n}$ с.в., определенных на вероятностном пространстве $(T, \mathscr{T}, \tau)$, удовлетворяет условию (с) теоремы 33.

Тогда при любом $m \leqslant n$ справедливо неравенство

$$
\tau\left\{t \in T:\left\|\sum_{i=1}^{n} \xi_{i}(t) f_{i}\right\|_{m, \infty} \leqslant C_{1}^{\prime}(n(1+\ln m))^{1 / 2}\right\} \leqslant C_{2}^{\prime} m^{-q^{\prime}}
$$

u, значит,

$$
\mathrm{E}\left\|\sum_{i=1}^{n} \xi_{i} f_{i}\right\|_{m, \infty} \geqslant C_{3}^{\prime}(n(1+\ln m))^{1 / 2}
$$

с некоторыми константами $q^{\prime}=q^{\prime}(p)>0 u C_{j}^{\prime}=C_{j}^{\prime}(p, M)>0 \quad(j=1,2,3)$.

Из последней теоремы, определения нормы в пространстве Марцинкевича (см. раздел 2), а также соотношения (90) сразу же вытекает следующий результат.

СлеДСТВИЕ 9. Пусть $\varphi \in \Psi$. Тогда, если выполнены условия теоремы 36, mo

$$
\mathrm{E}\left\|\sum_{i=1}^{n} \xi_{i} f_{i}\right\|_{M(\varphi)} \geqslant A \sqrt{n} \max _{m=2, \ldots, n}\left\{\frac{\sqrt{\ln m}}{m \varphi(1 / m)}\right\}
$$

c некоторой константой $A>0$.

\section{9. Изоморфизмы между симметричными пространствами на $[0,1]$ и $[0, \infty)$}

Общая проблема существования изоморфизмов между симметричными пространствами на $[0,1]$ и на $[0, \infty)$ (отличными от $L_{p}$-пространств) впервые была поставлена Б. С. Митягиным в [82]. Этот и другие близкие вопросы интенсивно изучались в [38] (см. также [17]) на основе подхода, использующего конструкцию стохастического интеграла относительно симметризованного пуассоновского процесса. Здесь мы будем следовать идеям нашей работы [52], где был предложен технически значительно более простой подход, основанный на применении теоремы 25; заметим, что аналогичные аргументы применялись ранее в работе [34] в специальном случае пространств Лоренца $L_{p, q}$. Первая часть следующей теоремы усиливает результаты работы [38] об изоморфном 
вложении некоторого симметричного пространства на полуоси в данное симметричное пространство $X$ на $[0,1]$, заменяя условие нетривиальности нижнего индекса Бойда $X$ на более слабое условие ограниченности оператора $\mathscr{K}$. Вторая часть теоремы доказана в [83]. Ранее (см. [38; §8] или [17; с. 203]) аналогичное утверждение было известно лишь в случае, когда симметричное пространство $X$ имеет нетривиальные индексы Бойда.

Tеорема 37. (i) Если оператор $\mathscr{K}$ ограниченно действует в симметричном пространстве $X$, то $X$ содержит подпространство, изоморфное пространству $Z_{X}^{2}$.

(ii) Если симметричное пространство $X$ максимально или сепарабельно и оператор $\mathscr{K}$ ограниченно действует в $X$ и в $X^{\times}$, то пространства $X$ и $Z_{X}^{2}$ изоморфны.

ДоказАтельство. Пусть $(\Omega, \mathscr{P}):=\prod_{k=1}^{\infty}\left([0,1], \lambda_{k}\right)$ (как и ранее, $\lambda_{k}-$ мера Лебега). Тогда по теореме 25 линейный оператор

$Q x\left(t, \omega_{1}, \omega_{2}, \ldots\right)=\sum_{k=1}^{\infty} x_{k}\left(\omega_{k}\right) r_{k}(t), \quad$ где $x_{k}\left(\omega_{k}\right)=x\left(k-1+\omega_{k}\right) \quad\left(0 \leqslant \omega_{k} \leqslant 1\right)$, ограниченно действует из пространства $Z_{X}^{2}$ в пространство $X(\Omega \times[0,1])$. Кроме того, ввиду теоремы 9 (см. первое из неравенств (26)) существует такая константа $c>0$, что $\|Q f\|_{X(\Omega \times[0,1])} \geqslant c\|f\|_{Z_{X}^{2}}$. Тем самым, образ оператора $\operatorname{Im}(Q)$ как подпространство в $X(\Omega \times[0,1])$ изоморфен пространству $Z_{X}^{2}$. Так как пространства $X$ и $X(\Omega \times[0,1])$ изометричны, то утверждение (i) доказано.

Перейдем к доказательству (ii). Покажем, что образ $\operatorname{Im}(Q)$ оператора $Q$, состоящий из всех функций $g \in X(\Omega \times[0,1])$, представимых в виде суммы ряда

$$
g\left(t, \omega_{1}, \omega_{2}, \ldots\right)=\sum_{k=1}^{\infty} g_{k}\left(\omega_{k}\right) r_{k}(t)
$$

который сходится п. в. на $\Omega \times[0,1]$, дополняем в пространстве $X(\Omega \times[0,1])$.

Определим на $L_{1}(\Omega \times[0,1])$ последовательность условных математических ожиданий

$$
\mathrm{E}\left(g \mid \omega_{k}\right)\left(\omega_{k}\right):=\left(\int_{0}^{1} \int_{0}^{1} \ldots\right) g\left(t, \omega_{1}, \omega_{2}, \ldots\right) d t d \omega_{1} \cdots d \omega_{k-1} d \omega_{k+1} \cdots \quad(k \geqslant 1),
$$

а также оператор

Обозначим

$$
P g\left(t, \omega_{1}, \omega_{2}, \ldots\right):=\sum_{k=1}^{\infty} \mathrm{E}\left(g r_{k} \mid \omega_{k}\right) r_{k}(t)
$$

$$
\langle f, g\rangle:=\left(\int_{0}^{1} \int_{0}^{1} \ldots\right) f\left(t, \omega_{1}, \omega_{2}, \ldots\right) g\left(t, \omega_{1}, \omega_{2}, \ldots\right) d t d \omega_{1} d \omega_{2} \ldots
$$

Непосредственная проверка показывает, что для произвольных $x, y \in L_{2}(0, \infty)$

$$
\langle Q x, Q y\rangle=\int_{0}^{\infty} x(t) y(t) d t
$$


и что оператор $P$ самосопряжен, т. е. для любых $f, g \in L_{2}(\Omega \times[0,1])$

$$
\langle P f, g\rangle=\langle f, P g\rangle \text {. }
$$

Докажем, что $P$ ограничен в $X(\Omega \times[0,1])$. Для этого сначала проверим, что для любой $f \in L_{\infty}(\Omega \times[0,1])$

$$
x(t):=\sum_{k=1}^{\infty} \mathrm{E}\left(f r_{k} \mid \omega_{k}\right)(t+k-1) \chi_{[k-1, k)}(t) \in L_{\infty} \cap L_{2}(0, \infty) .
$$

Во-первых, легко видеть, что $\left|\mathrm{E}\left(f r_{k} \mid \omega_{k}\right)\right| \leqslant\|f\|_{\infty}(k=1,2, \ldots)$, откуда следует, что $x \in L_{\infty}$. Кроме того, ввиду очевидного неравенства

$$
\begin{aligned}
& \left(\int_{0}^{1} \cdots\left(\int_{0}^{1} f\left(u, \omega_{1}, \ldots, \omega_{k-1}, t, \omega_{k+1}, \ldots\right) r_{k}(u) d u\right) d \omega_{1} \cdots d \omega_{k-1} d \omega_{k+1} \cdots\right)^{2} \\
& \quad \leqslant \int_{0}^{1} \cdots\left(\int_{0}^{1} f\left(u, \omega_{1}, \ldots, \omega_{k-1}, t, \omega_{k+1}, \ldots\right) r_{k}(u) d u\right)^{2} d \omega_{1} \cdots d \omega_{k-1} d \omega_{k+1} \cdots,
\end{aligned}
$$

верного для произвольных $0 \leqslant t \leqslant 1$ и $k=1,2, \ldots$, а также неравенства Бесселя имеем:

$$
\begin{aligned}
& \|x\|_{L_{2}(0, \infty)}^{2}=\sum_{k=1}^{\infty} \int_{0}^{1}\left(\int_{0}^{1} \cdots f\left(u, \omega_{1}, \ldots, \omega_{k-1}, t, \omega_{k+1}, \ldots\right)\right. \\
& \left.\quad \times r_{k}(u) d u d \omega_{1} \cdots d \omega_{k-1} d \omega_{k+1} \cdots\right)^{2} d t \\
& \leqslant\left(\int_{0}^{1} \int_{0}^{1} \cdots\right) \sum_{k=1}^{\infty}\left(\int_{0}^{1} f\left(u, \omega_{1}, \omega_{2}, \ldots, \omega_{k}, \ldots\right) r_{k}(u) d u\right)^{2} d \omega_{1} d \omega_{2} \cdots d \omega_{k} \cdots \\
& \leqslant\left(\int_{0}^{1} \int_{0}^{1} \ldots\right) f\left(u, \omega_{1}, \omega_{2}, \ldots, \omega_{k}, \ldots\right)^{2} d u d \omega_{1} d \omega_{2} \cdots=\|f\|_{2}^{2} \leqslant\|f\|_{\infty}^{2} .
\end{aligned}
$$

Итак, (97) доказано, и, значит, $x \in Z_{X}^{2}$. При этом $P f=Q x$. Так как по условию $X$ сепарабельно или максимально, то, как легко проверить, соответствующим свойством будет обладать и пространство $Z_{X}^{2}$. Кроме того, $\left(Z_{X}^{2}\right)^{\times}=Z_{X \times}^{2}$. Ввиду другого условия теоремы $\mathscr{K}: X \rightarrow X$. Таким образом, по теореме 25 получаем:

$$
\begin{aligned}
\|P f\|_{X(\Omega \times[0,1])} & =\|Q x\|_{X(\Omega \times[0,1])} \leqslant C\|x\|_{Z_{X}^{2}} \\
& =C \sup \left\{\left|\int_{0}^{\infty} x(t) y(t) d t\right|:\|y\|_{Z_{X \times}^{2}} \leqslant 1\right\} .
\end{aligned}
$$

Так как оператор $\mathscr{K}$ ограничен также и в $X^{\times}$, то из соотношений (95), (96), и опять теоремы 25 (но уже примененной к $X^{\times}$) следует:

$$
\begin{aligned}
& \|P f\|_{X(\Omega \times[0,1])} \leqslant C \sup \left\{|\langle Q x, Q y\rangle|:\|y\|_{Z_{X^{\times}}^{2}} \leqslant 1\right\} \\
& \quad=C \sup \left\{|\langle P f, Q y\rangle|:\|y\|_{Z_{X^{\times}}^{2}} \leqslant 1\right\}=C \sup \left\{|\langle f, Q y\rangle|:\|y\|_{Z_{X^{\times}}^{2}} \leqslant 1\right\} \\
& \quad \leqslant C \sup \left\{\|Q y\|_{X^{\times}(\Omega \times[0,1])}:\|y\|_{Z_{X^{\times}}^{2}} \leqslant 1\right\}\|f\|_{X(\Omega \times[0,1])} \leqslant C C^{\prime}\|f\|_{X(\Omega \times[0,1])} .
\end{aligned}
$$


В итоге для всех $f \in L_{\infty}(\Omega \times[0,1])$ выполнено неравенство

$$
\|P f\|_{X(\Omega \times[0,1])} \leqslant C C^{\prime}\|f\|_{X(\Omega \times[0,1])} .
$$

Так как по условию $X$ сепарабельно или максимально, то, применяя стандартные рассуждения, заключаем, что проектор $P$ ограничен в этом пространстве. Кроме того, легко видеть, что его образ $\operatorname{Im}(P)$ совпадает с образом $\operatorname{Im}(Q)$ оператора $Q$. Следовательно, подпространство $\operatorname{Im}(Q)$ (которое ввиду уже доказанной первой части теоремы изоморфно $\left.Z_{X}^{2}\right)$ дополняемо в $X(\Omega \times[0,1])$, которое изоморфно $X$. С другой стороны, очевидно, что $X$ изоморфно дополняемому подпространству пространства $Z_{X}^{2}$. Так как $X \oplus X\left(\right.$ соответственно $Z_{X}^{2} \oplus Z_{X}^{2}$ ) изоморфно $X$ (соответственно $Z_{X}^{2}$ ), то в итоге, применяя метод декомпозиции Пелчинского [17; с. 172], получаем изоморфность $X$ и $Z_{X}^{2}$. Теорема доказана.

Следующий результат, показывающий тесную связь между стохастическим интегралом Пуассона (который является ключевым средством в конструкциях $[38 ; \S 8]$ и $[17 ; 2 . f])$ и оператором Круглова $\mathscr{K}$, доказан в [52].

Оператор стохастического интегрирования относительно симметризованного процесса Пуассона (обозначим его через $T$ ) определен на функциях, измеримых на полуоси $(0, \infty)[17 ;$ с. $205-206]$. Если $f$ - функция на $[0,1]$, то $T f:=T^{\prime} f-T^{\prime \prime} f$, где

$$
T^{\prime} f(u, v):=\sum_{k=1}^{\infty} \chi_{F_{k}^{\prime}}(u) \sum_{j=1}^{k} f\left(\varphi_{j}^{\prime}(v)\right) \quad(u, v \in[0,1]) .
$$

Здесь $\left\{F_{k}^{\prime}\right\}_{k \geqslant 0}$ - разбиение отрезка $[0,1]$ на попарно дизъюнктные подмножества, $\lambda\left(F_{k}^{\prime}\right)=1 /(e k !), k=0,1,2, \ldots$, a $\left\{\varphi_{j}^{\prime}\right\}_{j=1}^{\infty}-$ последовательность независимых с.в., равномерно распределенных на $[0,1]$. Подобным же образом определяется и функция $T^{\prime \prime} f$, при этом соответствующие последовательности $\left\{F_{k}^{\prime \prime}\right\}_{k \geqslant 0}$ и $\left\{\varphi_{j}^{\prime \prime}\right\}_{j=1}^{\infty}$ независимы относительно последовательностей $\left\{F_{k}^{\prime}\right\}_{k \geqslant 0}$ и $\left\{\varphi_{j}^{\prime}\right\}_{j=1}^{\infty}$ соответственно. Таким образом, функции $T^{\prime} f$ и $T^{\prime \prime} f$ одинаково распределены и независимы. В случае функций $f$, определенных на $(0, \infty)$, рассмотрим функции $f_{n}:=f \chi_{(n-1, n)}(n=1,2, \ldots)$. Тогда, если функции $b_{n}-$ независимые копии $f_{n}$, заданные на $[0,1]$, то по определению $T f:=\sum_{n=1}^{\infty} T f_{n}$,
где $T f_{n}$ - независимые копии $T b_{n}(n=1,2, \ldots)$.

С помощью оператора Круглова в [52] получена характеризация симметричных пространств $X$ таких, что оператор $T$ ограниченно действует из $Z_{X}^{2}$ в $X$. Это усиливает результаты [38; 8$]$ (см. также $[17 ; 2 . f])$, где было найдено достаточное условие: $\alpha_{X}>0$.

Теорема 38. Если $X$ - сепарабельное или максимальное симметричное пространство на [0,1], то оператор $T$ ограничен из $Z_{X}^{2}$ в $X$ тогда и только тогда, когда оператор $\mathscr{K}$ ограничен в $X$.

ДокАзАтельство. Пусть сначала оператор $T$ ограничен из $Z_{X}^{2}$ в $X$. Если $f$ определена на $[0,1]$, то последовательность $\left\{f\left(\varphi_{j}^{\prime}\right)\right\}_{j=1}^{\infty}$ из определения оператора $T$ состоит из независимых с. в., имеющих то же распределение, что и $f$. Следовательно (см. (33)), с.в. $T^{\prime} f$ и $\mathscr{K} f$ одинаково распределены и, значит, 
выполнено $\left\|T^{\prime} f\right\|_{X}=\|\mathscr{K} f\|_{X}$. Более того, ввиду независимости $T^{\prime} f$ и $T^{\prime \prime} f$ для любого $\tau>0$

$$
\begin{aligned}
\lambda^{2}\{(u, v):|T f(u, v)|>\tau\} & \geqslant \lambda^{2}\left\{(u, v):\left|T^{\prime} f(u, v)\right|>\tau \text { и } u \in F_{0}^{\prime \prime}\right\} \\
& =e^{-1} \lambda^{2}\left\{(u, v):\left|T^{\prime} f(u, v)\right|>\tau\right\}
\end{aligned}
$$

и, таким образом, согласно предложению $1\|\mathscr{K} f\|_{X}=\left\|T^{\prime} f\right\|_{X} \leqslant e\|T f\|_{X}$. Итак, если оператор $T: Z_{X}^{2} \rightarrow X$ ограничен, то ограничен и оператор $\mathscr{K}: X \rightarrow X$.

Наоборот, предположим, что $\mathscr{K}: X \rightarrow X$ ограничен. Если $f$ определена на $[0,1]$, то по предыдущему

$$
\|T f\|_{X}=\left\|T^{\prime} f-T^{\prime \prime} f\right\|_{X} \leqslant 2\|\mathscr{K} f\|_{X}
$$

Пусть теперь функция $f \in Z_{X}^{2}$ произвольна. Так как операторы $T^{\prime}$ и $T^{\prime \prime}$ положительны, то ввиду определения $T$ [17; с. 207] можно предположить, что $f$ неотрицательна. Обозначим $f_{n}:=f \chi_{(n-1, n)}(n=1,2, \ldots)$. Легко видеть, что существуют такие последовательности $\left\{g_{n}\right\}_{n=1}^{\infty}$ и $\left\{h_{n}\right\}_{n=1}^{\infty}$, что $f_{n}=g_{n}+h_{n}$, $g_{n} h_{n}=0$ и $\int_{n-1}^{n} g_{n}(t) d t=\int_{n-1}^{n} h_{n}(t) d t \quad(n=1,2, \ldots)$. Поэтому, если $f_{n}^{\prime}:=$ $g_{n}-h_{n}$, то $\left|f_{n}^{\prime}\right|=f_{n}$ и $\int_{n-1}^{n} f_{n}^{\prime}(t) d t=0, n=1,2, \ldots$.

Далее, пусть $b_{n}$ - определенные на $[0,1]$ независимые копии функций $f_{n}^{\prime}$ $(n=1,2, \ldots)$. По теореме 25 имеем

$$
\left\|\sum_{k=1}^{n} b_{k}\right\|_{X} \leqslant C\left\|\sum_{k=1}^{n} f_{k}^{\prime}\right\|_{Z_{X}^{2}}=C\left\|\sum_{k=1}^{n} f_{k}\right\|_{Z_{X}^{2}} .
$$

Кроме того, из определения $T$ [17; с. 207] следует, что $\left\{T f_{n}\right\}_{n=1}^{\infty}$ и $\left\{T b_{n}\right\}_{n=1}^{\infty}-$ две последовательности независимых симметрично распределенных с. в., а также что $T f_{n}$ и $T b_{n}$ одинаково распределены для любого $n=1,2, \ldots$. Поэтому ввиду (98), (99) и ограниченности оператора $\mathscr{K}$ в пространстве $X$

$$
\begin{aligned}
\left\|T\left(\sum_{k=1}^{n} f_{k}\right)\right\|_{X} & =\left\|\sum_{k=1}^{n} T f_{k}\right\|_{X}=\left\|\sum_{k=1}^{n} T b_{k}\right\|_{X}=\left\|T\left(\sum_{k=1}^{n} b_{k}\right)\right\|_{X} \\
& \leqslant 2\left\|\mathscr{K}\left(\sum_{k=1}^{n} b_{k}\right)\right\|_{X} \leqslant 2\|\mathscr{K}\|_{X \rightarrow X}\left\|\sum_{k=1}^{n} b_{k}\right\|_{X} \\
& \leqslant 2\|\mathscr{K}\|_{X \rightarrow X} C\left\|\sum_{k=1}^{n} f_{k}\right\|_{Z_{X}^{2}} \leqslant 2\|\mathscr{K}\|_{X \rightarrow X} C\|f\|_{Z_{X}^{2}} \quad(n=1,2, \ldots) .
\end{aligned}
$$

Так как $X$ сепарабельно или максимально, то, применяя стандартные аргументы, заключаем, что оператор $T: Z_{X}^{2} \rightarrow X$ ограничен. Теорема доказана.

Из теоремы 38 и теоремы 2.f.1(i) из [17] вытекает следующий результат.

СлЕДСТвИЕ 10. Если симметричное пространство $X$ сепарабельно или максимально и $X \in \mathbb{K}$, то образ оператора стохастического интегрирования относительно симметризованного процесса Пуассона изоморфен пространству $Z_{X}^{2}$. 


\section{0. Гильбертовы подпространства симметричных пространств, порожденные независимыми функциями}

Задача описания подпространств симметричных пространств, порожденных последовательностями независимых функций, рассматривалась во многих работах (см., например, [34], [39], [84]). Покажем, что при ее решении весьма эффективным образом может быть использовано свойство Круглова.

Пусть $X$ - симметричное пространство на $[0,1]$. Рассмотрим вопрос о том, каким условиям должно удовлетворять $X$, чтобы каждая последовательность независимых одинаково распределенных ненулевых функций из $X$ натягивала в нем гильбертово подпространство. Нетрудно показать [85; теорема 3.4], что это эквивалентно существованию $C>0$, для которого неравенство

$$
C^{-1}\left\|f_{1}\right\|_{X}\left(\sum_{k=1}^{\infty} a_{k}^{2}\right)^{1 / 2} \leqslant\left\|\sum_{k=1}^{\infty} a_{k} f_{k}\right\|_{X} \leqslant C\left\|f_{1}\right\|_{X}\left(\sum_{k=1}^{\infty} a_{k}^{2}\right)^{1 / 2}
$$

верно для любой такой последовательности $\left\{f_{k}\right\}_{k=1}^{\infty} \subset X$ и всех $\left(a_{k}\right)_{k=1}^{\infty} \in$ $l_{2}$. Левая часть (100) выполнена в произвольном симметричном пространстве $X$ [39; лемма 1, с. 52], так что с этой точки зрения интересна лишь его правая часть. Следующие необходимые условия для выполнения неравенства (100) перечислены в [39; с. 71]:

(а) пространство $X$ содержит пространство $\left(\exp L_{2}\right)_{\circ}$;

(b) $\int_{0}^{1} f_{1}(x) d x=0$;

(c) $f_{1} \in L_{2}$.

Первое из них - непосредственное следствие теоремы Родина и Семенова (см. начало раздела 7 или [66]): достаточно в качестве $f_{k}$ взять функции Радемахера. Так как $f_{k}$ одинаково распределены, то второе условие вытекает из оценки

$$
C n^{1 / 2} \geqslant\left\|\sum_{k=1}^{n} f_{k}\right\|_{X} \geqslant \mathrm{E}\left|\sum_{k=1}^{n} f_{k}\right| \geqslant\left|\mathrm{E} \sum_{k=1}^{n} f_{k}\right|=n\left|\mathrm{E} f_{1}\right| \quad(n \in \mathbb{N}) .
$$

Проверим условие (с). Для произвольной последовательности независимых одинаково распределенных функций $\left\{f_{k}\right\}_{k=1}^{\infty} \subset X$ положим: $g_{k}:=f_{2 k}-f_{2 k-1}$ $(k=1,2, \ldots)$. Тогда $g_{k}$ симметрично и одинаково распределены и независимы. Следовательно, для них также выполнено соотношение (100). Для каждого $a>0$ определим $g_{k, a}:=g_{k} \chi_{\left\{\left|g_{k}\right|<a\right\}}$ и $h_{k, a}:=g_{k}-2 g_{k, a}$. Ввиду симметричности $g_{k}$ функции $h_{k, a}$ и $g_{k}$ одинаково распределены. Следовательно,

$$
\mathrm{E}\left|\sum_{k=1}^{n} g_{k, a}\right|=\frac{1}{2} \mathrm{E}\left|\sum_{k=1}^{\infty}\left(g_{k}-h_{k, a}\right)\right| \leqslant \mathrm{E}\left|\sum_{k=1}^{n} g_{k}\right| \leqslant C_{1} n^{1 / 2}
$$

Положим $\sigma^{2}(a):=\mathrm{E} g_{1, a}^{2}$, и пусть $\gamma$ - гауссовская с. в., $\mathrm{E} \gamma=0, \mathrm{E} \gamma^{2}=1$. Тогда по центральной предельной теореме [50] $\lim _{n \rightarrow \infty} \mathrm{E}\left|n^{-1 / 2} \sum_{k=1}^{n} g_{k, a}\right|=\sigma(a) \mathrm{E}|\gamma|$, 
откуда ввиду предыдущего неравенства находим, что $\sigma(a) \mathrm{E}|\gamma| \leqslant C_{1}$ для каждого $a>0$. Переходя к пределу при $a \rightarrow \infty$, получаем: $\mathrm{E} g_{1}^{2}<\infty$, а значит, и $\mathrm{E} f_{1}^{2}<\infty$.

Чтобы сформулировать первый результат об условиях, достаточных для выполнения (100), нам понадобятся некоторые обозначения из [39; § 3.2]. Для произвольных числовой последовательности $a=\left(a_{k}\right)_{k=1}^{\infty}$ и измеримой функции $f$ на $[0,1]$ положим

$$
Q_{a} f(t)=\sum_{k=1}^{\infty} \lambda\left\{s \in[0,1]:\left|a_{k} f(s)\right|>t\right\}, \quad t>0 .
$$

Говорят, что функция $f$ имеет свойство $A_{2}(X)\left(f \in A_{2}(X)\right)$, если для каждой $a \in \ell_{2}$ пространство $X$ содержит все функции $g$, удовлетворяющие условию: $\lambda\{s \in[0,1]:|g(s)|>t\} \leqslant C Q_{a} f(t)(t>0)$ для некоторого $C>0$.

Теорема 39 [39; теорема 3.7]. Если симметричное пространство X имеет свойство Круглова $u\left\{f_{k}\right\}_{k=1}^{\infty}-$ такая последовательность независимых одинаково распределенных функицй, что $f_{1} \in A_{2}(X), f_{1} \in L_{2} u \int_{0}^{1} f_{1}(x) d x=0$,
то выполнено (100).

Доказательство последнего утверждения в [39; 33.2$]$ основывается на теории безгранично делимых распределений и весьма длинно и сложно. В то же время, нетрудно видеть, что теорема 39 - непосредственное следствие ранее доказанной теоремы 25 (подробнее см. [85]). Здесь мы докажем другой результат работы [85], демонстрирующий эффективность операторного подхода к решению подобного рода задач: будет сформулировано простое интерполяционное условие, гарантирующее справедливость вложения $X \subset A_{2}(X)$.

TеОремА 40. Пусть $X$ - симметричное пространство на $[0,1]$, интерполяиионное между пространствами $L_{2} u L_{\infty}, X \in \mathbb{K}$. Тогда для некоторого $C>0$ и всех последовательностей независимых одинаково распределенных функций $\left\{f_{k}\right\}_{k=1}^{\infty} \subset X$ таких, что $\int_{0}^{1} f_{1}(x) d x=0$, и произвольньх $\left(a_{k}\right)_{k=1}^{\infty} \in \ell_{2}$
выполнено $(100)$.

ДокАЗАТЕльство. Для произвольной фиксированной последовательности $a=\left(a_{k}\right)_{k=1}^{\infty} \in l_{2}$ определим линейный оператор $T_{a}: S(0,1) \rightarrow S(0, \infty)$ формулой

$$
T_{a} f(t)=\sum_{k=1}^{\infty} a_{k} f(t-k+1) \chi_{(k-1, k]}(t) .
$$

Так как для каждой функции $f \in L_{2}$ (соответственно $\left.f \in L_{\infty}\right)\left\|T_{a} f\right\|_{2}=$ $\|a\|_{2}\|f\|_{2}$ (соответственно $\left.\left\|T_{a} f\right\|_{\infty}=\sup _{k}\left|a_{k}\right|\|f\|_{\infty} \leqslant\|a\|_{2}\|f\|_{\infty}\right)$, то ввиду равенств

$$
Z_{L_{2}{ }^{2}}=L_{2}(0, \infty) \quad \text { и } \quad Z_{L_{\infty}}^{2}=L_{\infty} \cap L_{2}(0, \infty)
$$

получаем, что

$$
\begin{array}{cl}
\left\|T_{a} f\right\|_{Z_{L_{2}}^{2}}=\|a\|_{2}\|f\|_{2} & \\
\left\|T_{a} f\right\|_{Z_{L_{\infty}}^{2}} \leqslant 2\|a\|_{2}\|f\|_{\infty} & \left(f \in L_{2}[0,1]\right),
\end{array}
$$


Иначе говоря, $T_{a}$ ограниченно действует из $L_{2}[0,1]$ в $Z_{L_{2}}^{2}$ и из $L_{\infty}[0,1]$ в $Z_{L_{\infty}}^{2}$. Для того чтобы “проинтерполировать" соотношения (102), распространив их на произвольное симметричное пространство $X \in \operatorname{Int}\left(L_{2}, L_{\infty}\right)$, нам потребуются две интерполяционные леммы, которые мы здесь только сформулируем (доказательство первой см. в [86; лемма 4], а второй - в [85; лемма 3.3]).

Лемма 18. Для произвольной банаховой пары $\left(X_{0}, X_{1}\right)$ и любого параметра $\Phi$ вещественного $\mathscr{K}$-метода справедливо следующее равенство:

$$
\left(X_{0}, X_{0} \cap X_{1}\right)_{\Phi}^{\mathscr{K}}=\left(X_{0}, X_{1}\right)_{\Phi}^{\mathscr{K}} \cap X_{0} .
$$

Так как пара $\left(L_{2}, L_{\infty}\right)$ является $\mathscr{K}$-монотонной [87], а $X \in \operatorname{Int}\left(L_{2}, L_{\infty}\right)$, то ввиду теоремы Брудного-Кругляка (см. раздел 2 или [22; теорема 3.3.20]) существует параметр $\Phi$ вещественного $\mathscr{K}$-метода, для которого

$$
X=\left(L_{2}, L_{\infty}\right)_{\Phi}^{\mathscr{K}}
$$

ЛЕмма 19. Если выполнено равенство (103), то с точностъю до эквивалентности норм

$$
Z_{X}^{2}(0, \infty)=\left(L_{2}(0, \infty), L_{\infty}(0, \infty)\right)_{\Phi}^{\mathscr{K}} \cap L_{2}(0, \infty) .
$$

Продолжим доказательство теоремы 40. Ввиду соотношений (102), а также лемм 18 и 19 существует константа $C_{1}>0$, зависящая лишь от пространства $X$, такая, что для произвольных $f \in X$ и $a \in \ell_{2}$

$$
\left\|T_{a} f\right\|_{Z_{X}^{2}} \leqslant C_{1}\|a\|_{2}\|f\|_{X}
$$

С другой стороны, так как $X \in \mathbb{K}$ по условию, то мы можем применить теорему 25. Поэтому для произвольной последовательности независимых функций $\left\{f_{k}\right\}_{k=1}^{n} \subset X$ таких, что $\int_{0}^{1} f_{k}(x) d x=0(k=1,2, \ldots)$, и любых $n \in \mathbb{N}$ и $a_{1}, \ldots, a_{n} \in \mathbb{R}$

$$
\left\|\sum_{k=1}^{n} a_{k} f_{k}\right\|_{X} \leqslant \beta\|\mathscr{K}\|_{X \rightarrow X}\left\|\sum_{k=1}^{n} a_{k} \mathbf{f}_{k}\right\|_{Z_{X}^{2}}
$$

где $\beta>0$ - некоторая универсальная константа. Так как функции $f_{k}(k=$ $1,2, \ldots)$ одинаково распределены, то аналогичное свойство имеют и функции $\sum_{k=1}^{n} a_{k} \mathbf{f}_{k}$ и $T_{a^{n}} f_{1}$, где $a^{n}=\left(a_{k}^{n}\right), a_{k}^{n}=a_{k}(k \leqslant n)$ и $a_{k}^{n}=0(k>n)$. Таким образом, из (104) и (105) вытекает, что

$$
\left\|\sum_{k=1}^{n} a_{k} f_{k}\right\|_{X} \leqslant C_{1} \beta\|\mathscr{K}\|_{X \rightarrow X}\left\|f_{1}\right\|_{X}\|a\|_{2}, \quad n=1,2, \ldots
$$

Справедливость правой оценки в (100) следует теперь из стандартных рассуждений. 
СлеДСтвиЕ 11. Пусть $X$ - такое симметричное пространство на $[0,1]$, что неравенство (100) выполнено для любой последовательности одинаково распределенных независимых функиий $\left\{f_{k}\right\}_{k=1}^{\infty} \subset X \operatorname{ma\kappa их,~что~} \int_{0}^{1} f_{1}(x) d x=0$. Тогда существует такая константа $C>0$, что для произвольной последовательности одинаково распределенных дизгюнктных функиий $\left\{g_{k}\right\}_{k=1}^{n} \in X$, $\left\|g_{1}\right\|_{X}=1$, любых $n \in \mathbb{N} u a_{k} \in \mathbb{R}(k=1, \ldots, n)$ справедливо следующее неравенство:

$$
\left\|\sum_{k=1}^{n} a_{k} g_{k}\right\|_{X} \leqslant C\left(\sum_{k=1}^{n} a_{k}^{2}\right)^{1 / 2} .
$$

В частности,

$$
\left\|\sum_{k=1}^{n} a_{k} \chi_{[(k-1) / n, k / n]}\right\|_{X} \leqslant C \phi_{X}\left(\frac{1}{n}\right)\left(\sum_{k=1}^{n} a_{k}^{2}\right)^{1 / 2} .
$$

ДокАЗАтельство. Нетрудно видеть, что существуют такие множества одинаково распределенных функций $\left\{g_{k}^{+}\right\}_{k=1}^{n}$ и $\left\{g_{k}^{-}\right\}_{k=1}^{n}$, что $\left|g_{k}\right|=g_{k}^{+}+g_{k}^{-}$, $g_{k}^{+} g_{k}^{-}=0$ и $\int_{0}^{1} g_{k}^{+}(x) d x=\int_{0}^{1} g_{k}^{-}(x) d x(k=1, \ldots, n)$. Тогда функции $g_{k}^{\prime}:=g_{k}^{+}-g_{k}^{-}$ $(k=1, \ldots, n)$ одинаково распределены и $\int_{0}^{1} g_{1}(x) d x=0$. Пусть $\left\{f_{k}\right\}_{k=1}^{n}-$ последовательность независимых копий функций $g_{k}^{\prime}$. Тогда $\left\|f_{1}\right\|_{X}=\left\|g_{1}^{\prime}\right\|_{X}=$ $\left\|g_{1}\right\|_{X}=1$, и по теореме 40 для произвольных $a_{1}, \ldots, a_{n} \in \mathbb{R}$

$$
\left\|\sum_{k=1}^{n} a_{k} f_{k}\right\|_{X} \leqslant C\left(\sum_{k=1}^{n} a_{k}^{2}\right)^{1 / 2}
$$

где константа $C>0$ зависит от $X$. Кроме того, по первой части теоремы 9 (см. также [39; лемма 5, с. 14-15]) мы получаем:

$$
\left\|\sum_{k=1}^{n} a_{k} f_{k}\right\|_{X} \geqslant \frac{1}{4}\left\|\sum_{k=1}^{n} a_{k} g_{k}^{\prime}\right\|_{X}=\frac{1}{4}\left\|\sum_{k=1}^{n} a_{k} g_{k}\right\|_{X} .
$$

Неравенство (106) следует из (108) и (109), а (107) - частный случай (106).

СЛЕДСтвИЕ 12. Если симметричное пространство $X \stackrel{\not=}{\subset} L_{2} u \phi_{X}(u)=u^{1 / 2}$, то существует последовательность одинаково распределенных независимых функций $\left\{f_{k}\right\}_{k=1}^{\infty} \subset X, \int_{0}^{1} f_{1}(x) d x=0$, которая натягивает подпространство в $X$, не изоморфное $\ell_{2}$.

ДокАзАТЕЛьство. Предположим, что каждая последовательность одинаково распределенных независимых функций $\left\{f_{k}\right\}_{k=1}^{\infty} \subset X, \int_{0}^{1} f_{1}(x) d x=0$, натягивает в $X$ подпространство, изоморфное $\ell_{2}$. Тогда ввиду замечания, сделанного в самом начале раздела, а также следствия 11 выполнено неравенство (107), эквивалентное следующему:

$$
\left\|\sum_{k=1}^{n} a_{k} \chi_{[(k-1) / n, k / n]}\right\|_{X} \leqslant C\left\|\sum_{k=1}^{n} a_{k} \chi_{[(k-1) / n, k / n]}\right\|_{L_{2}}
$$


где $n \in \mathbb{N}$ и $a_{k} \in \mathbb{R}(k=1, \ldots, n)$ произвольны. Отсюда немедленно следует включение $L_{2} \subseteq X$. Так как одновременно по условию следствия $X \subseteq L_{2}$, то $X=L_{2}$, а это противоречит другому условию.

ЗАмечание 12 . В частности, если $X=L_{2, q}(1 \leqslant q<2)$ (определение см. в разделе 2), то ввиду следствия 12 существует последовательность одинаково распределенных независимых функций $\left\{f_{k}\right\}_{k=1}^{\infty} \subset X, \int_{0}^{1} f_{1}(x) d x=0$, натягивающая в $X$ подпространство, не изоморфное $\ell_{2}$. Тем самым, приведенные в начале раздела условия (а)-(c) на симметричное пространство $X$ не достаточны для того, чтобы неравенство (100) было выполнено для каждой последовательности одинаково распределенных независимых функций $\left\{f_{k}\right\}_{k=1}^{\infty} \subset X$, $\int_{0}^{1} f_{1}(x) d x=0$. Это дает отрицательный ответ на вопрос, поставленный в книге $[39 ;$ с. 71$]$.

\section{Список литературы}

[1] H. P. Rosenthal, "On the subspaces of $L_{p}(p>2)$ spanned by sequences of independent random variables", Israel J. Math., 8:3 (1970), 273-303.

[2] В. Ф. Гапошкин, “Лакунарные ряды и независимые функции”, УМH, 21:6 (1966), 3-82; англ. пер.: V.F. Gaposhkin, "Lacunary series and independent functions", Russian Math. Surveys, 21:6 (1966), 1-82.

[3] Г. Пешкир, А.Н. Ширяев, "Неравенства Хинчина и мартингальное расширение сферы их действия", УМH, 50:5 (1995), 3-62; англ. пер.: G. Peshkir, A. N. Shiryaev, "The Khintchine inequalities and martingale expanding of the sphere of their action", Russian Math. Surveys, 50:5 (1995), 849-904.

[4] С. В. Асташкин, "Функции Радемахера в симметричных пространствах", Функциональный анализ, СМФН, 32, РУДН, М., 2009, 3-161; англ. пер.: S. V. Astashkin, "Rademacher functions in symmetric spaces", J. Math. Sci., 169:6 (2010), 725-886.

[5] С. В. Асташкин, Д. В. Занин, Е. М. Семенов, Ф. А. Сукочев, "Оператор Круглова и операторы, определяемые случайными перестановками", Функи. анализ и его прил., 43:2 (2009), 3-18; англ. пер.: S. V. Astashkin, D. V. Zanin, E. M. Semenov, F. A. Sukochev, "Kruglov operator and operators defined by random permutations", Funct. Anal. Appl., 43:2 (2009), 83-95.

[6] S. Kwapien, C. Schütt, "Some combinatorial and probabilistic inequalities and their applications to Banach space theory", Studia Math., 82:1 (1985), 91-106.

[7] C. Schütt, "Lorentz spaces that are isomorphic to subspaces of $L_{1}$ ", Trans. Amer. Math. Soc., 314:2 (1989), 583-595, http://www.jstor.org/stable/2001398.

[8] M. Junge, Q. Xu, "Noncommutative Burkholder/Rosenthal inequalities. II: Applications", Israel J. Math., 167:1 (2008), 227-282.

[9] A. Khintchine, "Über dyadische Brüche", Math. Z., 18:1 (1923), 109-116.

[10] С. Качмаж, Г. Штейнгауз, Теория ортогональных рядов, Физматгиз, М., 1958, 507 с.; пер. с нем.: S. Kaczmarz, H. Steinhaus, Theorie der Orthogonalreihen, Subwencji Funduszu Kultury Narodowej, Warszawa, 1935, 298 pp.

[11] J. Marcinkiewicz, A. Zygmund, "Remarque sur la loi du logarithme itéré", Fund. Math., 29 (1937), 215-222. 
[12] Б. С. Кашин, А. А. Саакян, Ортогоналъные рядъ, 2-е изд., Изд-во АФЦ, М., 1999, ISBN: 5-93379-003-6, 550 с.; англ. пер. 1-го изд.: В. S. Kashin, А. A. Saakyan, Orthogonal series, Trans. Amer. Math. Soc., 75, Amer. Math. Soc., Providence, RI, 1989, ISBN: 0-8218-4527-6, 451 pp.

[13] A. N. Kolmogoroff, "Über das Gesetz des iterierten Logarithmus", Math. Ann., 101:1 (1929), 126-135; рус. пер.: "О законе повторного логарифма", А. Н. Колмогоров, Теория вероятностей и математическая статистика, Наука, М., 1986, 34-44; перепеч.: А. Н. Колмогоров, Избранные трудь, 2, Наука, М., 2005, 37-46.

[14] Ю.В.Прохоров, "Одна экстремальная задача теории вероятностей”, Теория вероятн. и ее примен., 4:2 (1959), 211-214; англ. пер.: Yu. V. Prokhorov, "An extremal problem in probability theory", Theory Probab. Appl., 4 (1959), 201-203.

[15] Р. Эллиотт, Стохастический анализ и его приложения, Мир, М., 1986, 361 с.; пер. с англ.: R. J. Elliott, Stochastic calculus and applications, Appl. Math. (N. Y.), 18, Springer-Verlag, New York, 1982, ISBN: 0-387-90763-7, 302 pp.

[16] С. Г. Крейн, Ю.И. Петунин, Е. М. Семенов, Интерполяиия линейных операторов, Наука, M., 1978, 400 с.; англ. пер.: S. G. Krein, Yu. I. Petunin, E. M. Semenov, Interpolation of linear operators, Transl. Math. Monogr., 54, Amer. Math. Soc., Providence, RI, 1982, ISBN: 0-8218-4505-7, 375 pp.

[17] J. Lindenstrauss, L. Tzafriri, Classical Banach spaces. II. Function Spaces, Ergeb. Math. Grenzgeb., 97, Springer-Verlag, Berlin-New York, 1979, ISBN: 3-540-08888-1, 243 pp.

[18] C. Bennett, R. Sharpley, Interpolation of operators, Pure Appl. Math., 129, Academic Press, Boston, MA, 1988, ISBN: 0-12-088730-4, 469 pp.

[19] Л.В. Канторович, Г.П. Акилов, Функциональный анализ, 2-е изд., Наука, М., 1977, 741 с.; англ. пер. 1-го изд.: L. V. Kantorovich, G. P. Akilov, Functional analysis in normed spaces, International Series of Monographs in Pure and Applied Mathematics, 46, Macmillan, New York, 1964, 771 pp.

[20] М. А. Красносельский, Я. Б. Рутицкий, Выпуклье функции и пространства Орлича, Физматгиз, М., 1958, 271 с.; англ. пер.: M. A. Krasnosel'skii, Ja. B. Rutickii, Convex functions and Orlicz spaces, Noordhoff, Groningen, 1961, 249 pp.

[21] Й. Берг, Й. Лёфстрём, Интерполяиионные пространства. Введение, Мир, М., 1980, 264 c.; пер. с англ.: J. Bergh, J. Löfstróm, Interpolation spaces. An introduction, Grundlehren Math. Wiss., 223, Springer-Verlag, Berlin-New York, 1976, 207 pp.

[22] Ju. A. Brudnyi, N. Ya. Krugljak, Interpolation functors and interpolation spaces, v. I, North-Holland Math. Library, 47, North-Holland, Amsterdam, 1991, ISBN: 0-444-88001-1, 718 pp.

[23] A.P. Calderón, "Spaces between $L^{1}$ and $L^{\infty}$ and the theorem of Marcinkiewicz", Studia Math., 26 (1966), 273-299.

[24] Б. С. Митягин, "Интерполяционная теорема для модулярных пространств", Maтем. сб., 66:4 (1965), 473-482.

[25] A.P. Calderon, "Intermediate spaces and interpolation, the complex method", Studia Math., 24:2 (1964), 113-190.

[26] Г. Я. Лозановский, “Замечание об одной интерполяционной теореме Кальдерона", Функи. анализ и его прил., 6:4 (1972), 89-90; англ. пер.: G. Ya. Lozanovskii, "A remark on an interpolational theorem of Calderon", Funct. Anal. Appl., 6:4 (1972), 333-334.

[27] P. Hitczenko, "Domination inequality for martingale transforms of a Rademacher sequence", Israel J. Math., 84:1-2 (1993), 161-178.

[28] T. Holmstedt, "Interpolation of quasi-normed spaces", Math. Scand., 26:1 (1970), 177-199.

[29] S. J. Montgomery-Smith, "The distribution of Rademacher sums", Proc. Amer. Math. Soc., 109:2 (1990), 517-522. 
[30] Ж.-П. Кахан, Случайнье функииональные ряды, Мир, М., 1973, 304 с.; пер. с англ.: J.-P. Kahane, Some random series of functions, D. C. Heath and Co., Raytheon Education Co., Lexington, MA, 1968, 184 pp.

[31] R. Latala, "Estimation of moments of sums of independent real random variables", Ann. Probab., 25:3 (1997), 1502-1513.

[32] E. D. Gluskin, S. Kwapien, "Tail and moment estimates for sums of independent random variables with logarithmically concave tails", Studia Math., 114:3 (1995), 303-309.

[33] P. Hitczenko, S. J. Montgomery-Smith, K. Oleszkiewicz, "Moment inequalities for sums of certain independent symmetric random variables", Studia Math., 123:1 (1997), 15-42.

[34] N. L. Carothers, S. J. Dilworth, "Inequalities for sums of independent random variables", Proc. Amer. Math. Soc., 104:1 (1988), 221-226.

[35] W. B. Johnson, G. Schechtman, "Sums of independent random variables in rearrangement invariant function spaces", Ann. Probab., 17:2 (1989), 789-808.

[36] J. Hoffman-Jørgensen, "Sums of independent Banach space valued random variables", Studia Math., 52 (1974), 159-186.

[37] M. B. Marcus, G. Pisier, "Characterization of almost surely continuous $p$-stable random Fourier series and strongly stationary processes", Acta Math., 152:1 (1984), 245-301.

[38] W. B. Johnson, B. Maurey, G. Schechtman, L. Tzafriri, Symmetric structures in Banach spaces, Mem. Amer. Math. Soc., 19, № 217, 1979, 298 pp.

[39] M. Sh. Braverman, Independent random variables and rearrangement invariant spaces, London Math. Soc. Lecture Note Ser., 194, Cambridge Univ. Press, Cambridge, 1994, ISBN: 0-521-45515-4, 116 pp.

[40] В. М. Круглов, “Замечание к теории безгранично делимых законов”, Теория вероятн. и ее примен., 15:2 (1970), 330-336; англ. пер.: V. M. Kruglov, "A note on infinitely divisible distributions", Theory Probab. Appl., 15:2 (1970), 319-324.

[41] Е. Лукач, Характеристические функиии, Наука, M., 1979; пер. с англ.: Е. Lukacs, Characteristic functions, 2nd ed., Hafner, New York, 1970, 350 pp.

[42] S. V. Astashkin, F.A. Sukochev, "Series of independent random variables in rearrangement invariant spaces: An operator approach", Israel J. Math., 145:1 (2005), $125-156$.

[43] С.В. Асташкин, Ф.А. Сукочев, "Сравнение сумм независимых и дизъюнктных функций в симметричных пространствах", Матем. заметки, 76:4 (2004), 483-489; англ. пер.: S. V. Astashkin, F. A. Sukochev, "Comparison of sums of independent and disjoint functions in symmetric spaces", Math. Notes, 76:3-4 (1970), 449-454.

[44] S. V. Astashkin, F. A. Sukochev, "Best constants in Rosenthal-type inequalities and the Kruglov operator", Ann. Probab., 38:5 (2010), 1986-2008; arXiv: 1011.1381.

[45] S. Kwapień, W.A. Woyczyński, Random series and stochastic integrals: single and multiple, Probab. Appl., Birkhäuser, Boston, MA, 1992, ISBN: 0-8176-3572-6, 360 pp.

[46] V.I. Ovchinnikov, "The method of orbits in interpolation theory", Math. Rep., 1:2 (1984), 349-515.

[47] S. Montgomery-Smith, E. M. Semenov, "Random rearrangements and operators", Amer. Math. Soc. Transl. Ser. 2, 184, Amer. Math. Soc., Providence, RI, 1998, $157-183$.

[48] J. V. Ryff, "Orbits of $L_{1}$ functions under doubly stochastic transformations", Trans. Amer. Math. Soc., 117 (1965), 92-100, http://www.jstor.org/stable/1994198.

[49] V. I. Chilin, A. V. Krygin, F. A. Sukochev, "Extreme points of convex fully symmetric sets of measurable operators", Integral Equations Operator Theory, 15:2 (1992), $186-226$. 
[50] А.А. Боровков, Теория вероятностей, Наука, М., 1976, 432 с.; англ. пер. 2-го изд. 1986 г.: А. А. Borovkov, Probability theory, Gordon and Breach, Abingdon, Oxon, 1998, 474 pp.

[51] Ю. В. Прохоров, "Сильная устойчивость сумм и бесконечно делимых законов", Теория вероятн. и ее примен., 3:2 (1958), 153-165; англ. пер.: Yu. V. Prokhorov, "Strong stability of sums and infinitely divisible distributions", Theory Probab. Appl., 3:2 (1958), 141-153.

[52] С. В. Асташкин, Ф. А. Сукочев, "Ряды независимых функций с нулевым средним в симметричных пространствах со свойством Круглова", Исследования по линейным операторам и теории функций, Зап. научн. сем. ПОМИ, 345, 2007, 25-50; англ. пер.: S. V. Astashkin, F. A. Sukochev, "Series of independent, mean zero random variables in rearrangement-invariant spaces having the Kruglov property", J. Math. Sci. (N. Y.), 148:6 (2008), 795-809.

[53] F. A. Sukochev, D. V. Zanin, "Khintchine inequalities in quasi-normed spaces", manuscript.

[54] Y. Gordon, A. Litvak, C. Schütt, E. Werner, "Orlicz norms of sequences of random variables", Ann. Probab., 30:4 (2002), 1833-1853.

[55] Y. Gordon, A. Litvak, C. Schütt, E. Werner, "Geometry of spaces between polytopes and related zonotopes", Bull. Sci. Math., 126:9 (2002), 733-762.

[56] P. Hitczenko, S. Montgomery-Smith, "Measuring the magnitude of sums of independent random variables", Ann. Probab., 29:1 (2001), 447-466.

[57] S. Montgomery-Smith, "Rearrangement invariant norms of symmetric sequence norms of independent sequences of random variables", Israel J. Math., 131:1 (2002), 51-60.

[58] M. Junge, "The optimal order for the $p$-th moment of sums of independent random variables with respect to symmetric norms and related combinatorial estimates", Positivity, 10:2 (2006), 201-230.

[59] S. V. Astashkin, E. M. Semenov, F. A. Sukochev, "Banach-Saks type properties in rearrangement-invariant spaces with Kruglov property", Houston J. Math., 35:3 (2009), 959-973.

[60] С. В. Асташкин, К.Е. Тихомиров, “О некоторых вероятностных аналогах неравенства Розенталя", Матем. заметки (в печати).

[61] S. V. Astashkin, F.A. Sukochev, "Symmetric quasi-norms of sums of independent random variables in symmetric function spaces with the Kruglov property", Israel J. Math. (to appear).

[62] А. В. Бухвалов, "Интерполяция линейных операторов в пространствах векторфункций и со смешанной нормой”, Сиб. матем. журн., 28:1 (1987), 37-51; англ. пер.: A. V. Bukhvalov, "Interpolation of linear operators in spaces of vector-valued functions and with mixed norm", Siberian Math. J., 28:1 (1987), 24-36.

[63] D. L. Burkholder, "A sharp inequality for martingale transforms", Ann. Probab., 7:5 (1979), 858-863.

[64] W. Johnson, G. Schechtman, J. Zinn, "Best constants in moment inequalities for linear combinations of independent and exchangeable random variables", Ann. Probab., 13:1 (1985), 234-253.

[65] С. В. Асташкин, "Экстраполяционные функторы на семействе шкал, порожденных вещественным методом интерполяции", Сиб. матем. журн., 46:2 (2005), 264-289; англ. пер.: S. V. Astashkin, "Extrapolation functors on a family of scales generated by the real interpolation method", Siberian Math. J., 46:2 (2005), 205-225.

[66] V. A. Rodin, E. M. Semyonov, "Rademacher series in symmetric spaces", Anal. Math., 1:3 (1975), 207-222. 
[67] С. В. Асташкин, "Независимые функции в симметричных пространствах и свойство Круглова", Матем. сб., 199:7 (2008), 3-20; англ. пер.: S. V. Astashkin, "Independent functions in rearrangement invariant spaces and the Kruglov property", Sb. Math., 199:7 (2008), 945-963.

[68] С. В. Асташкин, "Обобщенное неравенство Хинчина в симметричных пространствах", Функи. анализ и его прил., 42:2 (2008), 78-81; англ. пер.: S. V. Astashkin, "A generalized Khintchine inequality in rearrangement invariant spaces", Funct. Anal. Appl., 42:2 (2008), 144-147.

[69] В.И. Дмитриев, С.Г. Крейн, В.И. Овчинников, “Основы теории интерполяции линейных операторов", Геометрия линейных пространств и теория операторов, Ярославль, 1977, 31-74.

[70] S. V. Astashkin, "About interpolation of subspaces of rearrangement invariant spaces generated by Rademacher system", Int. J. Math. Math. Sci., 25:7 (2001), 451-465.

[71] S. J. Szarek, "On the best constant in the Khintchine inequality", Studia Math., 58:2 (1976), 197-208.

[72] С. В. Асташкин, М.Ш. Браверман, "Подпространство симметричного пространства, порожденное системой Радемахера с векторными коэффициентами", Oneраторные уравнения в функииональных пространствах, Изд-во ВГУ, Воронеж, 1986, 3-10.

[73] R. Salem, A. Zygmund, "Some properties of trigonometric series whose terms have random signs", Acta Math., 91:1 (1954), 245-301.

[74] M. B. Marcus, G. Pisier, Random Fourier series with applications to harmonic analysis, Ann. of Math. Stud., 101, Princeton Univ. Press, Princeton, NJ; Univ. Tokyo Press, Tokyo, 1981, ISBN: 0-691-08289-8; 0-691-08292-8, 151 pp.

[75] M. Ledoux, M. Talagrand, Probability in Banach spaces, Ergeb. Math. Grenzgeb. (3), 23, Springer-Verlag, Berlin, 1991, ISBN: 3-540-52013-9, 480 pp.

[76] B. Kashin, L. Tzafriri, "Lower estimates for the supremum of some random processes", East J. Approx., 1:1 (1995), 125-139.

[77] B. Kashin, L. Tzafriri, "Lower estimates for the supremum of some random processes. II", East J. Approx., 1:3 (1995), 373-377.

[78] С. В. Асташкин, "О выделении подсистем, 'мажорируемых' системой Радемахера", Матем. заметки, 65:4 (1999), 483-495; англ. пер.: S. V. Astashkin, "Extraction of subsystems 'majorized' by the Rademacher system", Math. Notes, 65:4 (1999), 407-417.

[79] П.Г. Григорьев, "Случайные линейные комбинации функций из $L_{1}$ ", Maтем. заметки, 74:2 (2003), 192-220; англ. пер.: P. G. Grigor'ev, "Random linear combinations of functions from $L_{1}$ ", Math. Notes, 74:1-2 (2003), 185-211.

[80] P. G. Grigoriev, "Estimates for norms of random polynomials", East J. Approx., 7:4 (2001), 445-469.

[81] П. Г. Григорьев, "Оценки норм случайных полиномов и их приложение", Матем. заметки, 69:6 (2001), 950-954; англ. пер.: P. G. Grigor'ev, "Estimates for norms of random polynomials and their application", Math. Notes, 69:5-6 (2003), 868-872.

[82] Б. С. Митягин, "Гомотопическая структура линейной группы банахова пространства", УМH, 25:5 (1970), 63-106; B. S. Mityagin, "The homotopy structure of the linear group of a Banach space", Russian Math. Surveys, 25:5 (1970), 59-103.

[83] S. V. Astashkin, "Rademacher series and isomorphisms of rearrangement invariant spaces on the finite interval and on the semi-axis", J. Funct. Anal., 260:1 (2010), 195-207.

[84] L. E. Dor, J. T. Starbird, "Projections of $L_{p}$ onto subspaces spanned by independent random variables", Compositio Math., 39:2 (1979), 141-175. 
[85] S. V. Astashkin, F. A. Sukochev, "Sequences of independent identically distributed functions in rearrangement invariant spaces", Function spaces VIII, Banach Center Publ., 79, Polish Acad. Sci. Inst. Math., Warsaw, 2008, 27-37.

[86] С.В. Асташкин, "Об интерполяции пересечений вещественным методом”, Алгебра и анализ, 17:2 (2005), 33-69; англ. пер.: S. V. Astashkin, "Interpolation of intersections by the real method", St. Petersburg Math. J., 17:2 (2006), 239-265.

[87] G. G. Lorentz, T. Shimogaki, "Interpolation theorems for the pairs of spaces $\left(L^{p}, L^{\infty}\right)$ and $\left(L^{1}, L^{q}\right)$ ", Trans. Amer. Math. Soc., 159 (1971), 207-221.

C. В. Асташкин (S. V. Astashkin)

Самарский государственный университет

E-mail: astashkn@ssu.samara.ru

Ф. А. Сукочев (F. A. Sukochev)

School of Mathematics and Statistics,

University of New South Wales, Kensington, Australia

E-mail: f.sukochev@unsw.edu.au
Поступила в редакцию 21.06.2010 